\title{
TRIDENT-CTR User's Manual
}

\author{
Thomas J. Seed
}

This report was prepred as account of $w$ wh sponsored by the United Stales Government. Nesthet a... Uniled States nor the United Siates Department of

Energy, nor any of their employees. nor any of their

contractors, subcontuactors, of their employees, miakes

any warranty, express or implied, or assumes any legal

tiability or responstbility for the accuracy, completeness

of usefulness of any information, apparatus, product or

process disclosed, or represents that its use would not

infringe privalely owned rights.

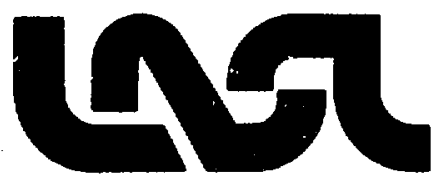


CONTENTS

ABSTRACT .

I. INTKODUCTION . . . . . . . . . . . . . . . . . . . . . . . . . 1

A. Basic Methodology . . . . . . . . . . . . . . . . . . . . . 1

B. Salient Features. . . . . . . . . . . . . . . . . . . . . o

1. Problem Size. . . . . . . . . . . . . . . . . . . 6

2. Flexible Mesh ..................... . . 7

3. Automatic Mesh Generator . . . . . . . . . . . . . . . 7

4. Fictitious Source Option . . . . . . . . . . . . . . . . . 7

5. Group Dependent Quadrature $\left(\mathrm{S}_{\mathrm{N}}\right)$. . . . . . . . . . . . . . 8

6. Internal Boundary Source Option . . . . . . . . . . . . . . 8

C. Basic Code Structure . . . . . . . . . . . . . . . . . . 8

D. Running On The NMFEC . . . . . . . . . . . . . . . . . . . . . . 9

1. Retrieve, Load, and Execute . . . . . . . . . . . . . 10

2. Modify CODEM . . . . . . . . . . . . . . . . . . . . . 12

E. Graphics Postprocessors . . . . . . . . . . . . . . . . . . 13

II. DATA INPUT . . . . . . . . . . . . . . . . . . . . . . . . . . . 14

A. Job Title Cards... . . . . . . . . . . . . . . . . 15

B. Control Integers . . . . . . . . . . . . . . . . . . . . 16

C. Control Floating-Point Numbers . . . . . . . . . . . . . . . . 20

D. Problem-Dependent Data . . . . . . . . . . . . . . . . . 20

E. Edit Input . . . . . . . . . . . . . . . . . . . . . . 27

F. Plot Edit Input... . . . . . . . . . . . . . . . . . 29

III. DETAILED INPUT DESCRIPTION . . . . . . . . . . . . . . . . . . . 31

A. Card One, Entry One ITH . . . . . . . . . . . . . . . . . . . 32

B. Card One, Entry Two ISCI . . . . . . . . . . . . . . . . . . . . 33

C. Card One, Entry Three IGEOM . . . . . . . . . . . . . . . . . . 33

D. Card One, Entry Four IGM . . . . . . . . . . . . . . . . . . . . 33

E. Card One, Entry Five IFS . . . . . . . . . . . . . . . . . . . . 34

F. Card One, Entry Six IEVT . . . . . . . . . . . . . . . . . . . . 35

G. Card One, Entry Seven ISTART . . . . . . . . . . . . . . . 38

H. Card One, Entry Eight ISTOP . . . . . . . . . . . . . . . . . . . 40

I. Card One, Entry Nine IQOPT . . . . . . . . . . . . . . . . . . . 41

J. Card One, Entry Ten NQG . . . . . . . . . . . . . . . . . . . 42

K. Card One, Eatry Eleven NBSG... . . . . . . . . . . . . . . . . 42

L. Card One, Entry Twelve JSTART . . . . . . . . . . . . . . . . . . 42

M. Card Two, General Comments on Boundary Control Integers

Specified on Entries One through Eight . . . . . . . . . . . . . 43

N. Card Two, Entry Nine JT .. . . . . . . . . . . . . . . . . 46

0. Card Two, Entry Ten ITMAX ... . . . . . . . . . . . . . . . . . 46

P. Card Two, Entry Eleven MSHOPT . . . . . . . . . . . . . . . . . . 47

Q. Card Three, General Comments on Internal Boundary Source Specification Integers on Entries One through Ten . . . . . . . 49

R. Card Four, Entry One MT............ . . . . . . . . . 50

S. Card Four, Entry Two MTPS . . . . . . . . . . . . . . . . . 50

T. Card Four, Entry Three MCR . . . . . . . . . . . . . . . . 52

U. Card Four, Entry Four MS . . . . . . . . . . . . . . . . 52 
V. Card Four, Entries Five through Seven . . . . . . . . . . . . . . 54

W. Card Four, Entry Eight IQAN ... . . . . . . . . . . . . . . . 54

X. Card Four, Entry Nine IPVT . . . . . . . . . . . . . . . . 55

Y. Card Four, Entry Ten ITTL . . . . . . . . . . . . . . . . . 56

Z. Card Five, Entries One through Five . . . . . . . . . . . . . . 56

AA. Card Five, Entries Six and Seven . . . . . . . . . . . . . . 57

BB. Card Six, Entry One ITLIM . . . . . . . . . . . . . . . . . . 58

CC. Card Six, Entry Two IEDOPT . . . . . . . . . . . . . . . . . . . 58

DD. Card Six, Entries Three through Seven . . . . . . . . . . . . 61

EE. Card Six, Entry Nine ISDB... . . . . . . . . . . . . . . . . . 61

FF. Card Six, Entry Eleven IDUMP . . . . . . . . . . . . . . . . . . 62

GG. Card Six, Entry Twelve ITTY . . . . . . . . . . . . . . . . . . . 62

HH. Card Seven, Entries One through Six.............. . . 62

II. Card Eight, Entry One EPSO .. . . . . . . . . . . . . . . . . 62

JJ. Card Eight, Entry Two NORM . . . . . . . . . . . . . . . . . . . 64

KK. Card Eight, Entry Three POD... . . . . . . . . . . . . . . . 65

LL. Entering the Spatial Mesh . . . . . . . . . . . . . . . . . . . . 65

MM. Entering the $\mathrm{S}_{\mathrm{N}}$ Specification and Constants . . . . . . . . . . . 68

NN. Entering the Source Specification Arrays . . . . . . . . . . . . 69

00. Entering the Cross Sections... . . . . . . . . . . . . . . . . 70

PP. Entering the Starting Fluxes . . . . . . . . . . . . . . . . . . 71

QQ. Entering the Inhomogeneous Distributed Source......... . . 73

RR. Entering the Boundary Sources . . . . . . . . . . . . . . . . . .

SS. Entering the Material Identification Array (IDCS) . . . . . . . . 78

TT. Entering Miscellaneous Group Dependent Arrays . . . . . . . . . . 78

UU. Entering the Cross Section Mixing Arrays (MIXNUM, MIXCOM, and MIXDEN) . . . . . . . . . . . . . . . . . . . . 79

VV. Entering the Buckling Height Array (BHT). . . . . . . . . . . . . 79

WW. Entering the Edit Input ... . . . . . . . . . . . . . . . . . . 79

XX. Entering the Plot Edit Input ................. . 81

IV. CODE STRUCTURE . . . . . . . . . . . . . . . . . . . . . . . . . . . . 82

REFERENCES . . . . . . . . . . . . . . . . . . . . . . . 108 
TRIDENT-CTR USER'S MANUAL

by

Thomas J. Seed

\begin{abstract}
TRIDENT-CTR is a two-dimensional, $x-y$ and $r-z$ geometry, multigroup, neucral particle transport code, developed for toroidal calculations. The use of triangular finite elements gives it the geometric flexibility to cope with the nonorthogonal shapes of many toroidal designs. This manual is intended to be a guide for the users of TRIDENT-CTR in making up the input required by the code, and not a description of the code theory and architecture.
\end{abstract}

\title{
I. INTRODUCTION
}

TRIDENT-CTR is a computer program for solving the two-dimensional neutral particle transport equation in rectangular $(x-y)$ and cylindrical $(r-z)$ geometries within a general domain having curved or other nonorthogonal boundaries. The spatial discretization is accomplished using triangular finite elements and discontinuous linear trial functions. TRIDENT-CTR is a modification of TRIDENT, ${ }^{1}$ a fission reactor code, written specifically for the fusion community. The code is capable of handling a wide variety of problems having irregular domains in both $x-y$ and $r-z$ geometries. However, many of the features in TRIDENT-CTR were incorporated to facilitate solution of large toroidal systems with nonregular poloidal cross sections. The use of triangles in $\mathrm{r}-\mathrm{z}$ geometry allows a user to accurately follow curved or irregularly shaped boundaries and material interfaces of toroidal shapes.

A. Basic Methodology

TRIDENT-CTR solves with suitable approximations the time-independent, inhomogeneous transport equation written below (also the homogeneous case).

$$
\underline{\nabla} \cdot(\underline{\Omega} \Psi)+\sigma_{t} \Psi(\underline{r}, E, \underline{\Omega})=\int \mathrm{dE}{ }^{\prime} \mathrm{d} \Omega^{\prime} \psi\left(\underline{r}, E^{\prime}, \underline{\Omega}^{\prime}\right) \sigma_{\mathrm{s}}\left(\mathrm{E}^{\prime} \rightarrow \mathrm{E}, \underline{\Omega}^{\prime} \cdot \underline{\Omega}\right)
$$




$$
\begin{aligned}
& +\chi(E) \int d E^{\prime} d \Omega^{\prime} \psi\left(\underline{x}, E^{\prime}, \underline{\Omega^{\prime}}\right) v \sigma_{\underline{f}}\left(E^{\prime}\right) / 4 \pi \\
& +Q(\underline{r}, E, \underline{\Omega}) .
\end{aligned}
$$

$\psi$ is the particle flux (particle number density times particle speed), defined such that $\psi d V d E d \Omega$ is the flux of particles in the volume element $d V$ about $\underline{r}$, in the element of solid angle $d \Omega$ about $\Omega$, in the energy range $d E$ about $E$. QdVdEd $\Omega$ is similarly defined for particles emitted by sources independent of $\psi \cdot \sigma_{t}, \sigma_{s}$, and $\sigma_{f}$ are respectively the macroscopic total interaction cross section, the macroscopic scattering transfer cross section, and the macroscopic fission cross section. v particles are emitted isotropically per fission with the fraction $\chi(E)$ within $d E$ about $E$.

TRIDENT-CTR uses the following standard approximations used in TWOTRAN, DOT, TRIDENT, and other 2-D transport codes.

(a) energy : multigroup approximation,

(b) angle : discrete ordinates approximation, and

(c) scattering transfer : Legendre polynomial expansion.

Making these approximations to Eq. (1) yields for $x$-y geometry:

$$
\mu_{m} \frac{\partial \psi_{m}^{g}(x, y)}{\partial x}+\eta_{m} \frac{\partial \psi_{m}^{g}(x, y)}{\partial y}+\sigma_{t}^{g} \psi_{m}^{g}(x, y)=s_{m}^{g}(x, y)
$$

and for $r-z$ geometry

$$
\begin{aligned}
& \frac{\mu_{m}}{r} \frac{\partial\left[\left(r \psi_{m}^{g}(r, z)\right]\right.}{\partial r}+\frac{\left[\alpha_{m+\frac{1}{2}} \psi_{m+\frac{1}{2}}^{g}(r, z)-\alpha_{m-\frac{1}{2}} \psi_{m-\frac{1}{2}}^{g}(r, z)\right]}{r w_{m}} \\
& \quad+\eta_{m} \frac{\partial \psi_{m}^{g}(r, z)}{\partial z}+\sigma_{t}^{g} \psi^{g}(r, z)=s_{m}^{g}(r, z) \\
& m=1,2, \ldots, M \quad g=1,2, \ldots, I G M,
\end{aligned}
$$

where $M$ is the total number of discrete ordinates directions and IGM is the number 
of energy groups in the multigroup approximation. $\mathrm{s}_{\mathrm{m}}^{\mathrm{g}}$ incorporates all inhomogeneous, fission, and scaticring sources. The path from Eq. (1) to Eqs. (2) and (3) is well covered in the manuals $1,2,3$ for the codes listed above.

To briefly describc ine spatial discretization method used in TRIDENT-CTR, consider the $x-y$ geometry equation with the group subscript ( $g$ ) suppressed. The spatial domain is triangulated using a banded structure as indicated in Fig. 1. The banded structure requires the vertices of all triangles be defined so that they fall on horizontal lines (lines perpendicular to the $y$ or $z$ axis). The restriction to a banded triangular mesh simplifies data input when compared to a general mesh. It also simplifies the mesh sweeping algorithm since for a given sign of $\eta,(\eta>0$ or $\eta<0)$, a horizontal line is immediately known to form an incoming or outgoing side to a band. Within the band a relatively simple algorithm is used to determine the order of sweeping the triangles so that the solution scheme is explicit. 4

Figure 1a illustrates the type of mesh required by TRIDENT. TRIDENT has the restriction that six and only six triangles share each internal node point. This arrangement insures that each side of a triangle corresponds to only one side of another triangle. When sweeping the mesh, particles exiting one triangle need not be apportioned among several other triangles as inflows. The TRIDENT restriction limits the difference between the number of triangles on adjacent bands to two triangles. This six-triangle restriction makes it difficult to specify a triangular mesh in regions of rapidly changing horizontal width. The more general mesh shown in Fig. Ib is allowed by TRIDENT-CTR. Relaxation of this six-triangle restriction

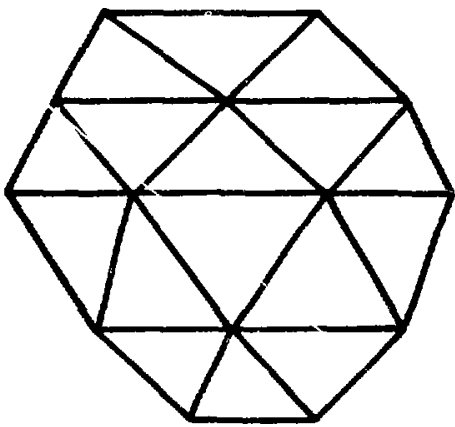

(a)

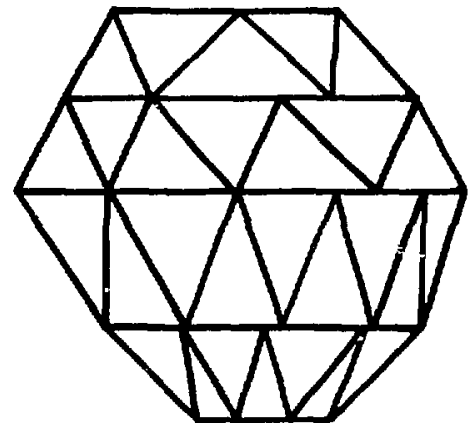

(b)

Fig. 1. TRIDENT-CTR meshes. 
allows much easier and more flexible specifications of the spatial mesh; however, it does require slightly more calculational effort to apportion particles.

The trial space used to approximate the flux consists of piecewise linear polynomials that are discontinuous across triangle boundaries, resulting in three unknowns per mesh cell. We assume that in triangle $i$

$$
\psi_{i}(x, y)=\sum_{k=1}^{3}: \psi_{i}^{k_{L} k}(x, y)
$$

With $L^{k}$ the linear Lagrange polynomial defined such that $L^{k}$ is unity at the $k^{\text {th }}$ triangle vertex $\left(x^{k}, y^{k}\right)$ and zero at the other two vertices. The directional subscript, $m$, has been suppressed. We thus allow three unknowns per triangle, these being the angular flux evaluated at the vertices. In $x-y$ geometry there are now three unknowns per triangle. Due to the angular derivative in $r-z$ geometry, there are six unknowns per triangle, although three of these are calculated by simple extrapolations since the diamond approximation is assumed in angle. ${ }^{5}$

The next task is to derive a set of equations equal to the number of unknowns. Because we are interested only in explicit methods, this task reduces, in $x-y$ geometry, to deriving a set of three equations for the three unknowns on a given triangle. The fluxes on incoming spatial boundaries are known from boundary conditions or from prior calculations in adjoining space cells. An incoming boundary for one cell is an outgoing boundary for an adjacent ceis or cells. The definition of incoming boundary depends upon the direction $\left(\mu_{m}, n_{m}\right)$ under consideration.

In TRIDENT-CTR adjacent triangles on the same band share only one side and particle flows within the band need not be apportioned among several triangles. As illustrated by Fig. 2, ihis is not true for flows between bands and a method

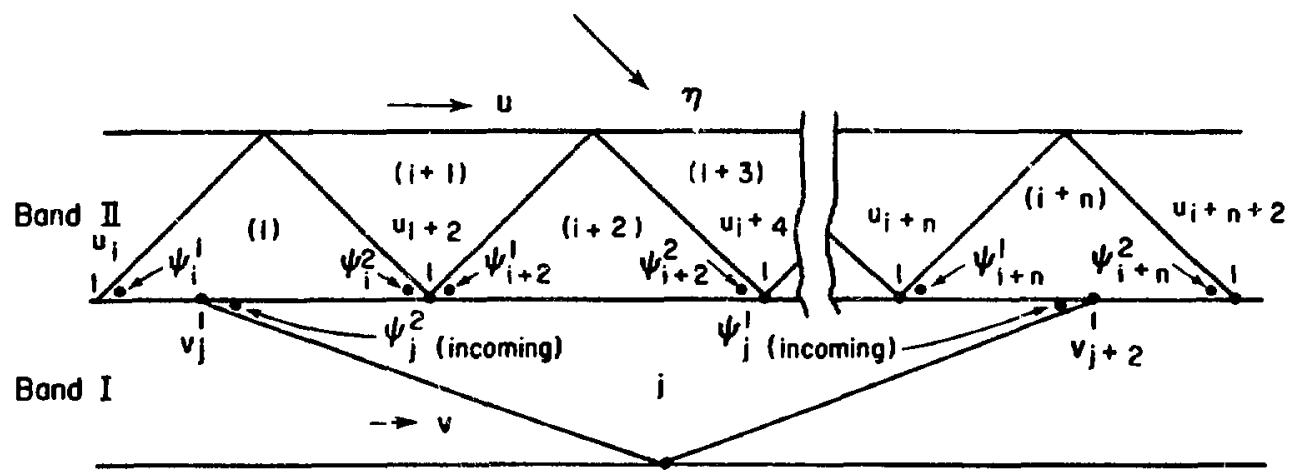

Fig. 2. Between-band interpolation 
for apportioning particle flows must be used. The apportionment or interpolation problem can be defined with reference to Fig. 2 as: given the angular fluxes $\left(\psi_{i}^{1}, \psi_{i}^{2}, \psi_{i+2}^{1}, \ldots, \psi_{i+n}\right)$ on band II for a downward direction; find the incoming angular flux for triangle $j\left[\psi_{j}^{1}\right.$ (incoming), $\left.\psi_{j \text { (incoming) }}^{2}\right]$ on band I. To simplify the expressions the horizontal coordinate ( $x$ ) will be changed to $v$ on band $I$ and $u$ on band II. To solve for these two unknowns, two equations must be derived. The natural constraints for establishing two equations are to conserve the neutron flow (1-moment) and the $x$-moment of the neutron flow between bands. Since TRIDENT-CTR solves for the angular flux by an expansion in piecewise linear trial functions, the angular flux at the band boundary may be written as

$$
\psi(x)=\psi^{1} L_{1}(x)+\psi^{2} L_{2}(x)
$$

where

$$
I_{1}(x)=\frac{\left(x-x_{2}\right)}{x_{1}-x_{2}}, L_{2}(x)=\frac{\left(x-x_{1}\right)}{x_{2}-x_{1}}
$$

Using Eqs. (5) and (6), a generai equation for the 1- and $x$-moment conservation equations may be written as

$$
\int_{v_{j}}^{v_{j+2}} v^{p} \sum_{k=1}^{2}\left[\psi_{j(\text { incoming })}^{k} L_{k}(v)\right] d v=\int_{v_{j}}^{v_{j+2}} u^{p} \sum_{\ell=i, i+2}^{i+n} \sum_{k=1}^{2} \psi_{\ell}^{k} L_{k}(u) d u
$$

$\mathrm{p}=0,1: \quad \mathrm{x}-\mathrm{y}$ geometry

$\mathrm{p}=1,2: \quad \mathrm{r}-\mathrm{z}$ geometry

Performing the integrations of Eq. (7) yields two equations which may be solved for $\psi_{j}^{1}$ (incoming) and $\psi_{j}^{2}$ (incoming)

We now have the known incoming fluxes. We insert Eq. (4) into Eq. (2) and weight by $1,(x-\bar{x})$, and $(y-\bar{y})$, where the barred coordinates refer to the triangle centroid. Integrating over the triangle areas gives three equations for the three 
unknowns. These equations are quite lengthy (especially for $r-z$ geometry) and are given in Ref. 4.

A special feature of TRIDENT that is also included in TRIDENT-CTR is rayeffert mitigation via the fictitious source fixup. Ray effects are nonphysical flus perturbations that occur in many two-dimensional discrete ordinates deeppenetration shielding problems. These fixups entail appending the within-group discrete ordinate equations of order $N$ with a fictitious source that ensures that the resulting equations satisfies spherical harmonic or spherical harmonic-like equations of order $\mathrm{N}-1$. The fictitious source is a complicated function of the angular flux. The Miller-Reed ${ }^{6}$ fictitious source is used in both codes.

Because of the complexities introduced by the removal of the vertex restrictions, the fine-mesh rebalance acceleration scheme used in TRIDENT is not used in TRIDENT-CTR. Whole system rebalance is used instead.

\section{B. Salient Features}

Although TRIDENT-CTR is a follow-on code to TRIDENT, it has incorporated several features that make it a significantly different code. As mentioned before, TRIDENT-CTR is capable of handling a wide range of irregular geometric domains in both $x-y$ and $r-z$ geometries. However, it was principally designed to solve shielding and blanket problems for large toroidal reactors, and the modifications incorporated into TRIDENT-CTR reflect that purpose. This verison of the code is primarily intended to be run at the Magnetic Fusion Energy (MFE) Computer Center on the center's $C D C-7600$ and the archicecture of the code also represents this intention. (A later version modified for the CRAY-1 is now under development.)

\section{Problem Size}

In TRIDENT, a requirement to hold in Small Core Memory (SCM, approximately 60000 words) all the information required to solve the entire spatial mesh for one group, limited the allowable problem size to approximately 3000 triangles in a $P_{0}$ calculation. This restriction would not allow solution of large toroidal problems with an adequate spatial mesh. To allow for larger problem sizes (a spatial mesh with a greater number of triangles), TRIDENT-CTR was designed to rcquire only the information to solve one band of triangles for one group to be in SCM at one time. The problem size is now constrained by Large Core Memory (LCM, approximately 400000 words). Rough estimates of typical problem sizes that TRIDENT-CTR can accommodate on a CDC-7600 are 36000 triangles for a $P_{0}$ 
calculation, 12000 triangles for a $P_{1}$ calculation, and 3600 triangles for a $P_{3}$ calculation. TRIDENT-CTR has the same energy-group blocking and overflow to disk as TRIDENT, and any practical number of energy groups may be used.

\section{Flexible Mesh}

As discussed in Sec. I.A, the TRIDENT mesh was difficult to form in regions of rapidly varying horizontal width. The six-triangle restriction made the number of triangles on one band dependent on the number of triangles on adjacent bands. In fact, the six-triangle restriction required that the maximum difference in the number of triangles between two adjacent bands be two. The TRIDENT-CTR mesh allows the number of triangles on any band to be completely independent of the number of triangles on adjacent bands. The TRIDENT-CTR mesh can easily follow rapid variations in the horizontal problem size. The decoupling of the number of triangles on adjacent bands also allowed the design of an efficient, easy to use mesh generator.

\section{Automatic Mesh Generator}

The TRIDENT-CTR mesh is flexible and fairly easy to specify in the sense of independence in placing vertices. However, in large problems the placing of thousands or tens of thousands of vertices would be an onerous task no matter how easy it is to place each vertex. To minimize the work involved in setting up a large problem, an automatic spatial mesh generator has been incorporated into TRIDENT-CTR. With the decoupling of bands discussed in Sec. I.B.2, the task of the automatic mesh generator is reduced to that of triangulating one band at a time. The automatic mesh generator takes input on a per band basis: the number of zones on a band, the number of triangles per zone, and the coordinates of the zone boundaries. A mesh generator zone is defined as a region of cunstant "card-image input" starting-flux guess, constant distributed inhomogeneous source, and constant material specification. The mesh generator basically works by attempting to form a rectangular area in the center of a zone and dividing it with isosceles triangles. Several variations of this procedure are used to prevent the appearance of "holes" in the mesh. A somewhat more detailed version of the mechanics of the mesh generator is contained in Sec. III.P.

\section{Fictitious Source Option}

As with TRIDENT, TRIDENT-CTR has an option to eliminate or mitigate ray-effect $^{7}$ distortions that plague some discrete-ordinates calculaticns. A fictitious source $^{6,8}$ is appended to the within-group-discrete-ordinates equations of order $N$ $\left(\mathrm{S}_{\mathrm{N}}\right.$ equations) that is defined to yield spherical-haromincs $\left(\mathrm{P}_{\mathrm{N}-1}\right)$ solutions in 
$x-y$ geometry and spherical-iarmonic-like $\left(\mathrm{DH}_{\mathrm{N}-1}\right)$ solutions in $\mathrm{r}-\mathrm{z}$ geometry. The addition of the fictitious source, which depends on the angular flux, results in a degradation of the convergence properties of the inner-iteration process, increasing the number of iterations by a factor of two or more depending on the problem. A set of parameters inputed when the fictitious source option is used may reduce this penalty in running time while mitigating but not completely eliminating the ray effect.

An effect similar to the ray effect occurs in $\mathrm{S}_{\mathrm{N}}$ solutions when neutrons are streaming through a vacuum. Severe nonphysical perturbations in the solution flux can occur, especially with isolated sources within a large vacuum region (i.e., tokamak plasmas). Unfortunately, ir problems where the total cross section is zero the fictitious source method degenerates to the $\mathrm{S}_{\mathrm{N}}$ solution. To address the neutron streaming problem, the following two options were incorporated in TRIDENT-CTR.

5. Group-Dependent Quadrature $\left(\mathrm{S}_{\mathrm{N}}\right)$

Valid arguments for including a group-dependent quadrature option can be made without consideration of the neutron streaming problem. In fact, other codes such as DOT-IV do have such an option available. However, the inclusion of the group-dependent $S_{N}$ option into TRIDENT-CTR was initially prompted by streaming considerations. The group-dependent $\mathrm{S}_{\mathrm{N}}$ option may be used to increase the $S_{N}$ order in those energy groups (e.g.,14-MeV source group) where the streaming effects are important, while retaining a reduced $\mathrm{S}_{\mathrm{N}}$ order in groups where streaming effects are minimal.

6. Internal Boundary Source Option

In addition to specifying an incoming boundary source on the exterior boundaries of the problem domain, TRIDENT-CTR allows the specification of boundary sources in the interior of the problem domain. A left, right, top, and bottom internal boundary source may be specified in any combination and along any boundaries corresponding to triangle sides. The internal boundary source option allows the user to replace a distributed neutron source in a central vacuum region with a deterministically (or other higher order method) calculated boundary source at the first material surface (i.e., the first wall).

C. Basic Code Structure

TRIDENT-CTR is a three-level overlayed code. The basic overlay structure is shown in Fig. 3. Each box in Fig. 3 contains the overlay identification, a one or two-word description of the overlay function, and the name of the code source 


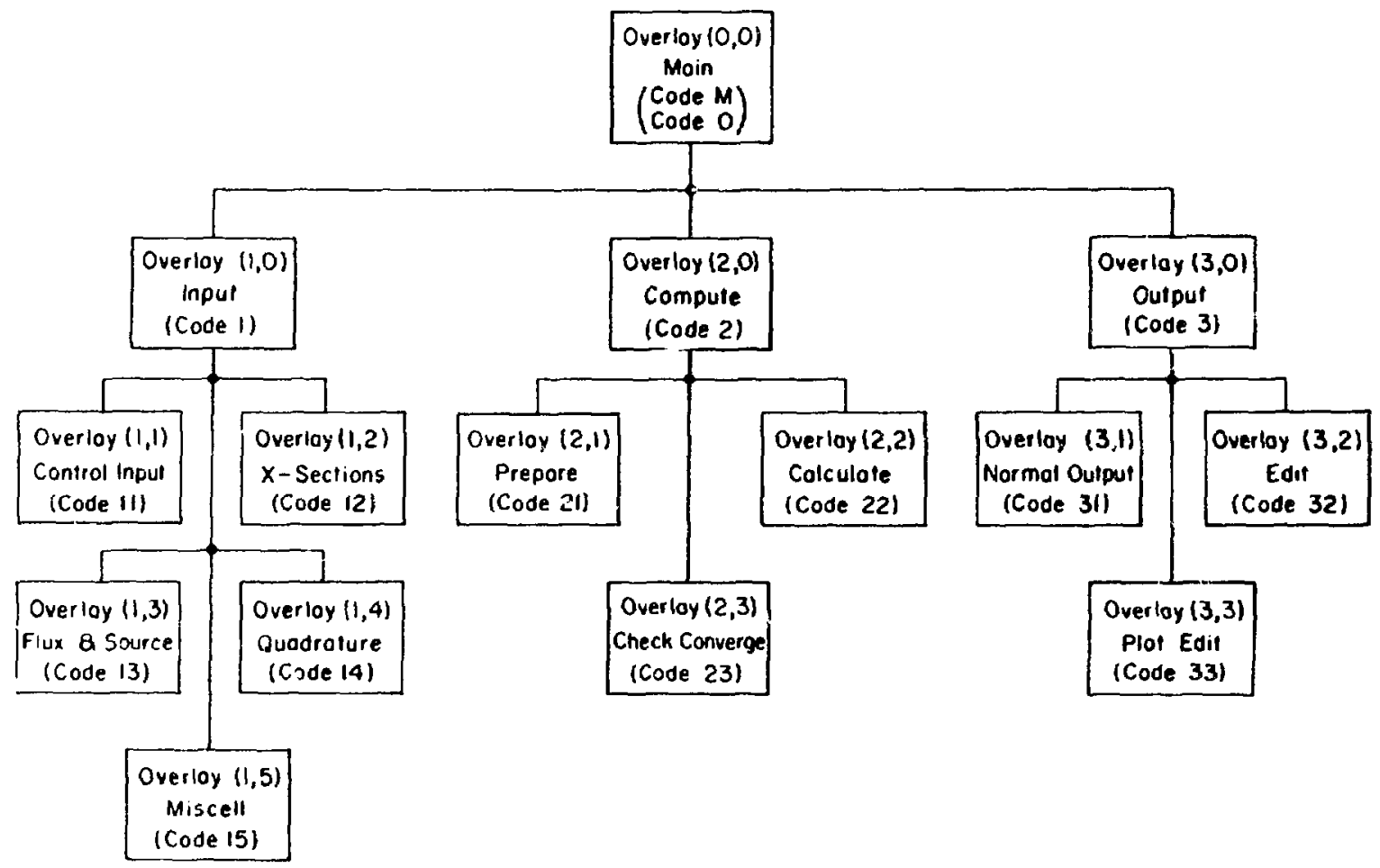

Fig. 3. TRIDENT-CTR Overlay Structure

listing (in parentheses). The main overlay source code has been broken in two parts. Code $M$ is a small source code that contains the control for the overlayed code execution and certain parameters such as the required SCM and LCM storage amounts. Changes in these parameters are made by changing them in the source code and recompiling.

The second level of the overlay structure is split into the three functional divisions of input, computation, and output. In the normal course of events, code $M$ is the only portion of the code which requires changes and recompiling by the user.

D. Running on the NMFECC

TRIDENT-CTR is installed at the National Magnetic Fusion Energy (NMFE) Computer Center at Livermore, California. It is available for use on the MFE CDC-7600. The CDC-7600 at Livermore has approximately 60000 words of storage in SCM and about 400000 words of LCM. Of the 60000 vords of SCM, about 30000 words are available for array space in TRIDENT-CTR. The descriptions for use of 
the code that foliow assume the user is familiar with the LTSS operating system and the utilities required for executing the code. For the new user, the utility DOCUMENT provides documentation for the LTSS operating system. The system utility routines the user must use to access, execute, and modify TRIDENT-CTR are listed below:

(1) FILEM - mass storage access,

(2) IIX - code library access,

(3) LOD - loading of the executable file,

(4) NeTOUT - MFE netwozk file transport,

(5) NETPLOT - IFE network graphics,

(6) TRIX AC - create input file and modify source code,

(7) $\mathrm{CH} / \mathrm{T}$ - compile source code, and

(8) LIBliAK - provide binary files suitable for use with LOD.

The TRIDENT-CTR source code and relocatable binaries are contained in two LIX library files named TRDCTRS and TRDCTRB. Messages to users and postprocessing codes are stored in the LIX files TRDCTRM and TRDCTRF. All four of these files may be obtained with the FILEM utility using an access code of 542 .

In all cases, execution of TRIDENT-CTR requires the existence of a card image (BCD) file labeled INPUT. The composition of this file is described in detail in Secs. II and III. The INPUT file may be created by using the TRIX AC utility and the user will find the DED data editor feature of this utility especially useful.

1. Retrieve, Load, and Execute

To run TRIDENT-CTR, the user must first obtain a copy of the code's relocatable binary file in his local file space. This is accomplished by using the following sequence of commands with the utility routine FILEM.

FILEM / $t \mathbf{v}$

.READ 542 TRDCTRB

.END

The associated TRIDENT-CTR files TRDCTRS, TRDCTRM, and TRDCTRP discussed above may also be obtained by the preceding procedure.

TRDCTRB is a LIX library file that contains a set of relocatable binaries for TRIDENT-CTR. There is a binary file corresponding to each overlay component of the code. The binaries are labeled as BINM, BINO, BIN1, BIN11, etc., corresponding to 
the overlay labeling of Fig. 3. Also containad in TRDCTRB is a file, labeled LODTRD, which contains the loading instructions that enable the LOD utility to produce an executable file from the relocatable binaries. A copy of the main overlay, CODEM, is included for user modification of code-dependent parameters such as LCM length. To unload the required files from TRDCTRB the following commands are used with the utility routine IIX.

\section{IIX $/ \mathrm{t} v$}

.GET ALL

.END

At this point required changes to CODEM may be made and a new BINM created. This procedure is described in Sec. I.D.2.

An executable file is obtained by using the following one line command with the LOD utility.

LOD XNN 10 LODTRD\% / $\mathrm{t} v$

(XNN is the user's box number)

Execution of the above line will produce an executable file of TRIDENT-CTR labeled TRDCTR. To execute the code, given that the file INPUT exists in the user's file space, the following line is used.

TRDCTR / $\mathrm{t} v$

The coie will then execute. The executing code will shange its name to $+\mathrm{CB} 00$ and run until the problem completes as instructed in the INPUT file, until a fatal error occurs (erroneous input) or until the time specified in the command line is used up. If the problem stops due to lack of time, it may be restarted by: 
$+\mathrm{CBOO} / \mathrm{t} \mathrm{v}$.

The code will print out cert ' n monitoring information at the terminal during execution. At problem completion, the output is contained in a file called TRDOUT. This file can be sent, to a desired location on the MFE network with the utility NETOUT .

\section{Modify CODEM}

There are four code limits that are set in CODEM that a user may want to modify. They are the SCM and LCM memory requirements, the maximum dump file length, and the time between periodic flux dumps.

To change the SCM requirements, two modifications must be made. The dimension of the array $A$ in blank common must be changed to the desired limit. Also the parameter MAXLEN must also be set to the desired number. MAXLEN sets the allowed array space in SCM and does not include the code overhead requirements. The maximum value that can be used for MAXLEN is something less than 30000 words.

To change the LCM requirements, changes in the dimensioning of arrays AALCM and BBLCM are made and the value assigned to MAXECS is changed. AALCM and BBLCM are contained in the labeled LCM common blocks $/ 1 /$ and $/ 2 /$. For LCM requirements less than 250000 , AALCM is dimensioned to the desired LCM memory requirements, BBLCM is dimensioned to one, and MAXECS is set tu the LCM memory requirement. For LCM requirements greater than 250000 , MAXECS is set to the desired numbe: AALCM is dimensioned to 250000 , and BBLCM is dimensioned to MAXECS-250 000 .

The maximum dump file length is set by the integer parameter MAXDMP and the time between periodic dumps is set in seconds with the floating point parameter TIMBDP.

Once the desired changes have been made, a binary file is created for use with the LOD loader. First the old binary file, BINM, is destroyed with the utility DESTROY.

DESTROY BINM / $\mathbf{t} \mathbf{v}$

CODEM is then compiled with the CHAT COMPILER. 
CHAT CODEM, C, TRDMS, LISTM, XNN / $t \mathrm{v}$.

The binary file created by CHAT is then put in a form compatible with LOD by the LIBMAK utility.

\section{LIBMAK C. TRDM BINM / $\mathrm{t} v$.}

\section{E. Graphics Postprocessors}

Contained in the LIX library file TRDCTRP are the source codes for the two graphics postprocessors TRD2D and TRD3D. The two postprocessor codes take as input binary data files created by TRIDENT-CTR. The specification for creation of the binary plot files is made in the INPUT file discussed in the following sections.

The TRD2D code produces two-dimensional plots of TRIDENT-CTR spatial meshes. Triangles are drawn and material identification numbers are placed in all triangles in which they can be fitted. The TRD2D code will display either the mesh across the entire problem domain or in a specified rectangular subregion. The input file for TRD2D is produced by TRIDENT-CTR and is labeled MSHiPLT. To compile and produce an executable file the following CHAT line is used.

CHAT (TV8OLIB, ORDERLIB, ) TRD2D, TRD2DE, TRD2DB, LIST, XNN, M, M / t v

The code is then executed by

TRD2DE / $\mathrm{t} v$.

While executing, the code queries the user for the plotting domain, and reply is made via the user's terminal. The code will produce several plots of the spatial mesh and terminate's on command by the user. 
The TRD3D code produces three-dimensional plots of the variation of quantities such as the flux vs spatial position. Inpul to the code is made from a binary file labeled EDTPLT. TRIDENT-CTR can produce up to three different three-dimensional plotting files labeled MATPLT, FLXPLT, and RESPLT. Fine contents of these files may be copied into a file labeled EDTPLT with the utility COPY or the label switched with the utility SWITCH. To produce an executable file from the source code the following CHAT line is used:

CHAT (TV80LIB, ORDERLIB, )TRD3D, TRD3DE, TRD3DB, L.IST, XNN, M, M / $t v$

The code is then executed by

TRD3DE $/ \mathrm{t} v$

Output from each of these graphirs codes will be in a file with a label of the form

DXmmddttt,

where mmdd is the month and day respectively and ttt ' $\fallingdotseq$ 's the time. This file may be processed with the NETPLOT utility routine.

\section{DATA INPUT}

In the following pages the input data for TRIDENT-CTR are listed in exactly the order in which they are entered in the code. The data are divided into four categories:

(1) job title cards,

(2) control integers on cards 1-6 and control floatingpoint numbers on cards 7 and 8 ,

(3) problem-dependent data on subsequent cards, and

(4) edit input. 
Except for the control parameters, cross sections, mesh input integers, and edit parameters, all floating-point numbers and integers are read into TRIDENTCTR in special formats by the LOAD subroutine. These formats are [6(I1,I2,E9.4], indicated by $S(E)$, for reading floating point numbers and $[6(I 1, I 2,19)]$, indicated by $S(I)$ for integers. In each word of both of these formats, the first integer field, Il, designates the options listed in Table $I$.

A special routine, PRINTS, is used to print some of the two- and three-dimensional arrays that occur in the program. This routine can be used for one-, two-, and three-dimensional arrays and has an option for printing a portion of an array (e.g., the mixed cross section blocks). When fluxes and fission rates are printed, they are oriented with the origin at the lower left of the page.

A. Job Title Cards

The user begins by indicating on a card in an 16 format the number of following title or job description cards he wants to use. He then enters the descriptive material on these cards, which are read with an $18 \mathrm{A4}$ format.

TABLE I

OPTIONS FOR SDECIAL LOAD FORMAT

$\frac{\text { Value of I1 }}{0 \text { or blank }}$

1

2

3

4

5

9
Nature of Option

No action.

Repeat data word in 9 field number of times indicated in 12 field.

Place number of linear interpolates indicated in 12 field between data word in 9 field and data word in next 9

field. Not allowed for integers.

Terminate reading of data block. A 3 must follow the last data word of all blocks.

Fill remainder of block with data word in 9 field. This operation must be followed by a terminate (3).

Repeat data word in 9 field 10 times the value in the I2 field.

Skip to the next data card. 
B. Control Integers

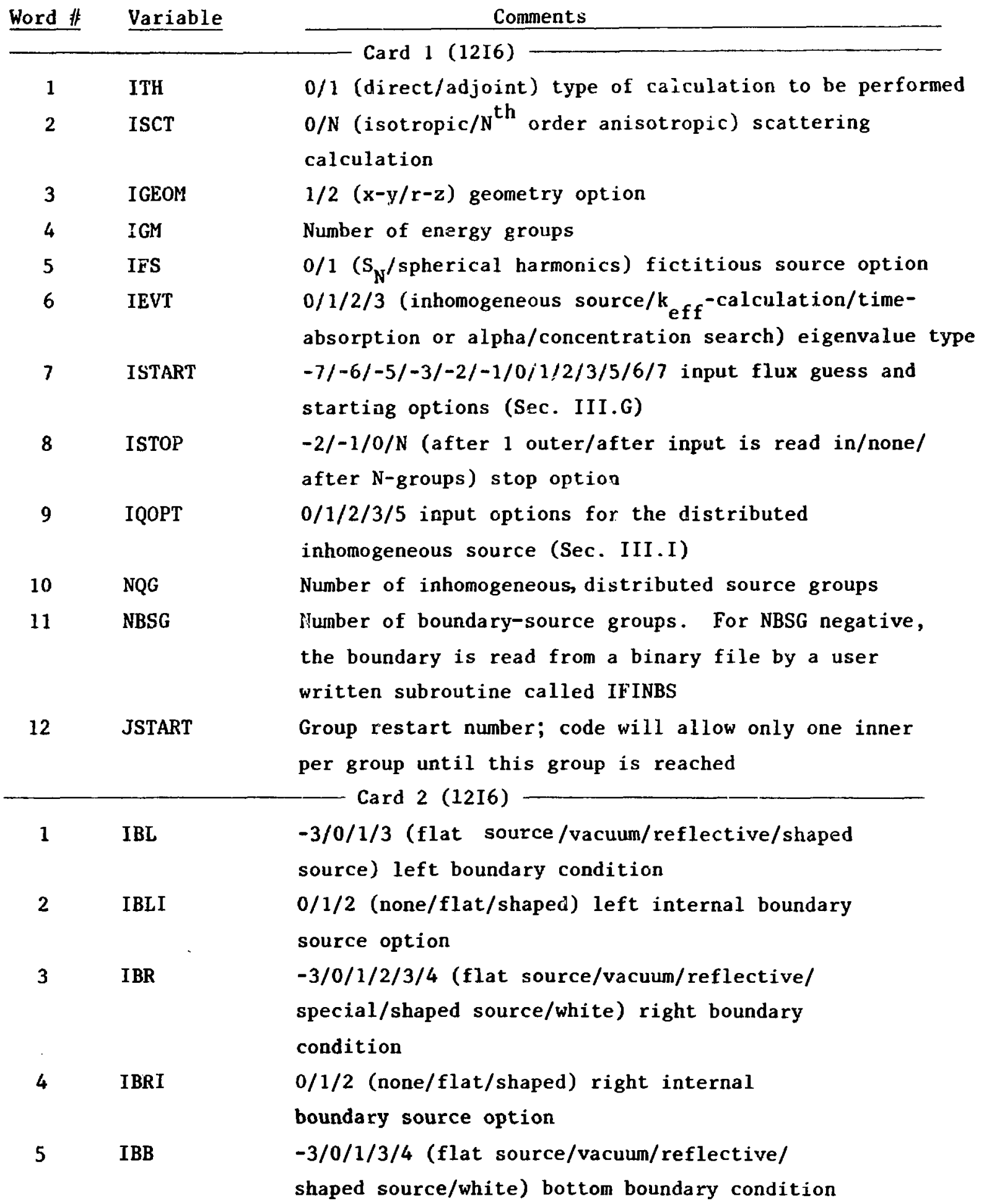




\begin{tabular}{|c|c|c|}
\hline Word I⿰ & Variable & Comments \\
\hline 6 & IBBI & $\begin{array}{l}0 / 1 / 2 \text { (none/flat/shaped) bottom internal boundary } \\
\text { source option }\end{array}$ \\
\hline 7 & IBT & $\begin{array}{l}-3 / 0 / 1 / 3 / 4 \text { (flat source/vacuum/shaped source/white) } \\
\text { top boundary condition }\end{array}$ \\
\hline 8 & ¿BTI & $\begin{array}{l}0 / 1 / 2 \text { (none/flat/shaped) top internal boundary } \\
\text { source option }\end{array}$ \\
\hline 9 & JT & Number of bands of triangles \\
\hline $1 \mathrm{C}$ & ITMAX & $\begin{array}{l}\text { Estimated maximum number of triangles on any one } \\
\text { band. (The automatic mesher can add up to three } \\
\text { triangles per zone for zones containing more than } \\
\text { one triangle.) }\end{array}$ \\
\hline 11 & MSHOPT & $\begin{array}{l}0 / 1 \text { (automatic mesher/input triangle vertices) } \\
\text { spatial mesh option } \\
\text { Card } 3 \text { (I } 216)\end{array}$ \\
\hline 1 & JLIB & $\begin{array}{l}\text { Band ID of the bottom band of the left internal } \\
\text { boundary source }\end{array}$ \\
\hline 2 & JLIT & $\begin{array}{l}\text { Band ID of the top band of the left internal } \\
\text { boundary source }\end{array}$ \\
\hline 3 & JRIB & $\begin{array}{l}\text { Band ID of the bottom band of the right } \\
\text { internal boundary source }\end{array}$ \\
\hline 4 & JRIT & $\begin{array}{l}\text { Band ID of the top band of the right internal } \\
\text { boundary source }\end{array}$ \\
\hline 5 & JBBI & $\begin{array}{l}\text { Band ID of the bottom internal boundary source band. } \\
\text { The boundary source is located at the top of } \\
\text { this band and is downward }(-\eta) \text { directed only }\end{array}$ \\
\hline 6 & NBBI & $\begin{array}{l}\text { Number of triangles in the bottom internal } \\
\text { boundary source band containing the source. } \\
\text { They must all be down-pointing and adjacent }\end{array}$ \\
\hline 7 & ITBBI & $\begin{array}{l}\text { Triangle ID of the first bottom internal } \\
\text { boundary source triangle }\end{array}$ \\
\hline 8 & JBTI & $\begin{array}{l}\text { Band ID of the top internal boundary source } \\
\text { band. The boundary source is located at the } \\
\text { bottom of this band and is upward directed } \\
(+n) \text { only }\end{array}$ \\
\hline
\end{tabular}




\begin{tabular}{|c|c|c|}
\hline Word \# & Variable & Comments \\
\hline 10 & ITBTI & $\begin{array}{l}\text { Number of triangles in the top internal boundary } \\
\text { source band containing the source. They must } \\
\text { all be up-pointing and arjacent } \\
\text { Triangle ID of the first top internal boundary } \\
\text { source triangle }\end{array}$ \\
\hline 1 & MT & $\begin{array}{l}\text { Total number of materials (cross section } \\
\text { ilocks, including anisotropic cross sections) } \\
\text { in the problem }\end{array}$ \\
\hline 2 & MTPS & $\begin{array}{l}-\mathrm{N} /+\mathrm{N} \text { (GPODXS/ISOTXS) number of materials } \\
\text { from interface file - CAUTION: Each } \\
\text { material set from this file yields ISCT }+1 \\
\text { materials }\end{array}$ \\
\hline 3 & MCR & $\begin{array}{l}\text { Number of input materials from code de- } \\
\text { pendent input file. If this number is } \\
\text { negative, FlDo-format cross sections are } \\
\text { read }\end{array}$ \\
\hline 4 & MS & $\begin{array}{l}\text { Number of mixture instructions. See } \\
\text { Sec. III. U and items MIXNUM, MIXCOM, } \\
\text { and MIXDEN below }\end{array}$ \\
\hline 5 & IHT & $\begin{array}{l}\text { Row of the total cross section in the } \\
\text { cross section format. If negative, the } \\
\text { code assumes there is no } \sigma^{\text {up in table }}\end{array}$ \\
\hline 6 & IHS & $\begin{array}{l}\text { Row of the within-group scattering cross } \\
\text { section in the cross section format }\end{array}$ \\
\hline 7 & IHM & $\begin{array}{l}\text { Total number of rows in the cross section } \\
\text { format }\end{array}$ \\
\hline 8 & IQAN & $\begin{array}{l}\text { Order of anisotropy of the inhomogeneous } \\
\text { source }\end{array}$ \\
\hline 9 & IPVT & $\begin{array}{l}0 / 1 / 2 \text { (none } / k_{\text {keff }} / \text { alpha) parametric eigen- } \\
\text { value entered. See entry PV }\end{array}$ \\
\hline 10 & IITL & $\begin{array}{l}\text { Maximum number of inner iterations allowed } \\
\text { per group }\end{array}$ \\
\hline
\end{tabular}




\begin{tabular}{|c|c|c|}
\hline 1 & MISN & $\begin{array}{l}\text { Maximum } S_{N} \text { order in any group. When ISNOPT=2, } \\
\text { MISN should be negative and equal to the total } \\
\text { number of directions to be read in }\end{array}$ \\
\hline 2 & MMNPQ & $\begin{array}{l}\text { Maximum number of directions in any one } \\
\text { quadrant in any one } S_{N} \text { set }\end{array}$ \\
\hline 3 & MMNEL & $\begin{array}{l}\text { Maximum number of eta levels in any one } \\
\text { quadrant in any one } S_{N} \text { set }\end{array}$ \\
\hline 4 & ISNOPT & $\begin{array}{l}-1 / 1 / 2 / 3 \text { (group dependent } \mathrm{S}_{\mathrm{N}} / \text { built-in/ } \\
\text { card input/interface file) quadrature set } \\
\text { optjons. Group dependent may be a mix of } \\
\text { cards and built-in }\end{array}$ \\
\hline 5 & NSN & $\begin{array}{l}\text { Number of } S_{N} \text { sets, must be } \geq 2 \text { if } \\
\text { ISNOPT }<0\end{array}$ \\
\hline 6 & IMSHPT & $\begin{array}{l}\text { 0/1 (no/yes) 2-D spatial mesh plot } \\
\text { binary file to be generated. A } \\
\text { binary file calied MSHPLT is produced } \\
\text { which may be used by a postprocessor } \\
\text { to produce a plot of the spatial mesh }\end{array}$ \\
\hline 7 & IEDPLT & $\begin{array}{l}0 / 1 \text { (no/yes) 3-D edit plot binary files } \\
\text { to be generated. Edit information will } \\
\text { be required in the problem-dependent } \\
\text { data section }\end{array}$ \\
\hline 1 & ITLIM & $\begin{array}{l}0 / \mathrm{N} \text { (no/ } \mathrm{N} \text {-second) time limit. If an integer } \\
\text { number of seconds is entered a restart dump } \\
\text { is taken after this number of seconds and } \\
\text { the problem is terminated }\end{array}$ \\
\hline 2 & IEDOPT & $0 / 1$ (no/yes) special edit input to be entered \\
\hline 3 & I1 & $\begin{array}{l}0 / 1 \text { (yes/no) full input flux-print suppression } \\
\text { indicator }\end{array}$ \\
\hline 4 & IFP & $\begin{array}{l}0 / 1 / 2 \text { (all/isotropic/none) final-flux print } \\
\text { indicator }\end{array}$ \\
\hline 5 & I3 & $\begin{array}{l}0 / 1 / 2 \text { (all/mixed/none) cross section print } \\
\text { indicator }\end{array}$ \\
\hline
\end{tabular}




\begin{tabular}{|c|c|}
\hline Word 非 & Variable \\
\hline 6 & 14 \\
\hline 7 & I5 \\
\hline 8 & I6 \\
\hline 9 & ISDB \\
\hline 10 & I7 \\
\hline 11 & IDUMP \\
\hline 12 & ITTY \\
\hline
\end{tabular}

$0 / 1$ (yes/no) final-fission print indicator

0/1/2/3 (all/unnormalized/normalized/none)

source-print indicator

Not: used, set to 0

0/: (whole system/space dependent) buckling

height indicator

Not used, set to zero

$0 / 1$ (scalar/total) final flux dump is created

0/1 (no/yes) TTY output option: available

c. Control Floating-Point Numbers

Word \# Variable

Comments

Card 7 (6E 12.5)

1 EV Eigenvalue guess. It is satisfactory to enter

0.0 for IEVT=2 and 1.0 for IEVT=3

2 EVM

Eigenvalue modifier. Used only if IEVT $>1$

$3 \quad \mathrm{PV}$

Parametric value of $k_{\text {eff }}$ for subcritical or

supercritical systems or for $1 / \mathrm{v}$ absorption

4 XLAL Lambda lower limit for eigenvalue searches

$5 \quad$ XIAH

Lambda upper limit for eigenvalue searches

6 XLAX

Search lambda convergence precision for second

and subsequent values of the eigenvalue

Card 8 (6E12.4)

1 EPSO Convergence precision

2 NORM

Normalization factor. Total number of source

or fission particles normalized to this number

if it is nonzero. No normalization if NORM

is zero

3 POD Parameter oscillation damper used in eigenvalue searches

D. Problem-Dependent Data

Block Name

\& Dimension

HY(JT)
Format $\begin{aligned} & \text { \# of } \\ & \text { Entries }\end{aligned}$

$\mathrm{S}(\mathrm{E}) \quad \mathrm{JT}$
Comments

Heights of triangle bands 


\begin{tabular}{|c|c|c|c|}
\hline $\begin{array}{l}\operatorname{ITZ}(\mathrm{J}) \\
\operatorname{ITT}(\mathrm{J})\end{array}$ & 216 & 2 & $\begin{array}{l}\text { Number of zones on the } j^{\text {th }} \text { band } \\
1 / 2 \text { (up/down) integers indicating } \\
\text { orientation of first triangle on } \\
\text { the } \mathrm{J}^{\text {th }} \text { band }\end{array}$ \\
\hline NTZ (ITMAX) & $12 I 6$ & $\operatorname{ITZ}(\mathrm{J})$ & Number of triangles per zone \\
\hline $\operatorname{BDZ}(2 *(\operatorname{ITMAX}+1))$ & $\mathbf{S}(\mathrm{E})$ & $2 *(\operatorname{ITZ}(J)+1)$ & $\begin{array}{l}x \text {-coordinates of zone boundaries } \\
\text { given from bottom to top, left } \\
\text { to right (i.e., lower coordinate, } \\
\text { upper coordinate, lower coordinate, } \\
\text { etc.) }\end{array}$ \\
\hline
\end{tabular}

For MESHOPT $=1$ load the following JT blocks of data

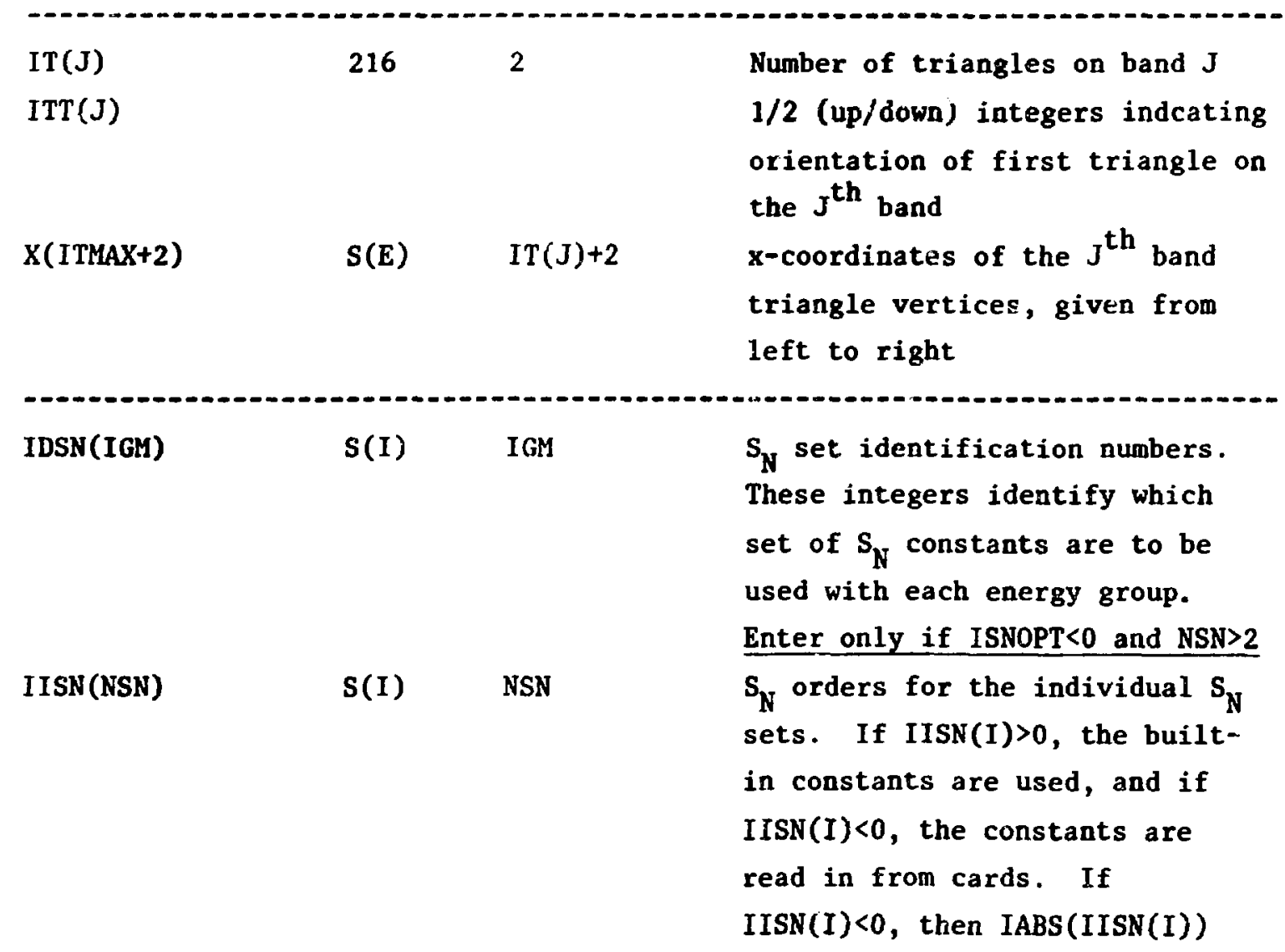


Enter the following sets of $S_{N}$ constants for as many values of IISN(I) that were negative. The first set will correspond to the first negative value of IISN(I) and second to the second negative value and so on. If ISNOPT $=2$, enter one set. $(M M=\operatorname{IABS}(\operatorname{IISN}(I))$

$\begin{array}{llll}\text { WGT (4*MMNP) } & \mathrm{S}(\mathrm{E}) & \mathrm{MM} & \mathrm{S}_{\mathrm{N}} \text { quadrature weights } \\ \operatorname{COSMU}(4 \times \mathrm{MMNP}) & \mathrm{S}(\mathrm{E}) & \mathrm{MM} & \mathrm{S}_{\mathrm{N}} \mu \text { cosine directions } \\ \operatorname{COSETA}(4 \times \mathrm{MMNPQ}) & \mathrm{S}(\mathrm{E}) & \mathrm{MM} & \mathrm{S}_{\mathrm{N}} \eta \text { cosine directions }\end{array}$

IDQ( IGM)

$\mathrm{S}(\mathrm{I})$

IGM

Inhomogeneous distributed source identification numbers. Enter only if IEVT $=0$ and NQG $>0$

IDBS ( IGM)

S(I) IGM

$\operatorname{IDBL}(\mathrm{JLT})$

S(I) JLT

$(\mathrm{JLT}=\mathrm{JLIT}-\mathrm{JLIB}+1)$

IDBR (JRT)

S(I) JRT

(JRT=JRIT-JRIB+1)

C(IHM, IGM, MIN)
Boundary source identification

numbers. Enter only if IEVT=0

and $\mathrm{NBSG}>0$

Triangle identification numbers

for the left internal boundary

source. The source will be

treated as entering from the

right side of the triangle

identified. Enter only if

NBSG $>0$ and IBLI $>0$

Triangle identification numbers

for the right internal boundary

source. The source will be treated as entering from the left side of the triangle identitied. Enter only if NBSG>0 and IBRI $>0$ Four options are available for reading cross sections. The LASL 
format may not be mixed with the FIDO format.

1. LASL INPUT. If MCR.GT.0, MCR Blocks of IHM*IGM numbers are read in a 6E12.5 format. Each block is preceded by an identification card read in an $18 \mathrm{~A} .4$ format. $M I N=M C R+$ MTPS $*($ ISCT +1$)$.

2. FIDO INPUT. If MCR.LT . 0, MCR b]ocks of data are created from FIDO input.

3. INTERFACE FILE ISOTXS.

When MTPS.GT.0, MTPS material

sets are read from standard file ISOTXS. On this file each material set consists of ISCT+1 cross section blocks one for isotropic and ISCT anisotropic cross sections. The first component of the material is stored in cross section block $M C R+1$, the first component of the second material is stored in cross section block MCR+ISCT+2, etc. Should the ISOTXS file not contain ISCT anisotropic components, zeroes are supplied for the compunents, not present. If the ISOTXS file contains more components than needed, oniy the first $\mathrm{ISCT}+1$ components are read. 4. FILE GRODXS. When MTPS.LT.0, a group-ordered cross section set of IABS(MTPS) materials is read in. 


\begin{abstract}
IDLIB (MTPS)
FLUX (NM , 3, ITMAX)

S(E)
\end{abstract}

MTPS

Option
This file is used when the entire cross section file (IHM IGM MIN) is too large to be continued in SCM. GPODXS is generated by th. LASL code MIXIT.

Position numbers of material sets to be read from ISOTXS. Do not enter unless MTPS.GT.0. The material sets need not be in order of increasing identification number. Number of entries depends on option. See Sec. III.00.

The input flux is entered on a per band basis with the number of entries dependent on whether the automatic mesh generator is used:

$$
\begin{array}{ll}
\text { for MESHOPT=0 } & \begin{array}{l}
\operatorname{IT} *=I T Z(J), \\
\text { fnumber of zones) }
\end{array} \\
\text { for MESHOPT }=1 & \begin{array}{l}
\operatorname{IT} *=3 * I T(J), \\
\text { (number of points) }
\end{array}
\end{array}
$$

\section{\# of Entries}

Problem restarts from three scalar flux binary files: RTFLUX, ATFIUX AND RAFLUX Problem restarts from two scalar flux binzry files RTFLUX and ATFLUX Problem restarts from scalar flux file RTFLUX

IGM entries (group spectrum), then JT bands of IT: (isotropic component) IGM groups of JT bands of IT\% (isotropic component) IGM (group spectrum) None NM sets of IGM (group spectrum) IGM groups of JT bands of NM sets of IT 
3

5

6

7

Block Name

\& Dimension

Q(NMQ , 3, ITMAX)
IGM entries (group spectrum), then JT bands of NM sets of IT*

Problem restarts from binary flux

file RTFLUX (a11 NM moments)

Problem restarts from two binary

flux flies RTFLUX and ATFLUX

Problem restart from three binary

flux files RTFLUX, ATFLUX, and

RAFLUX

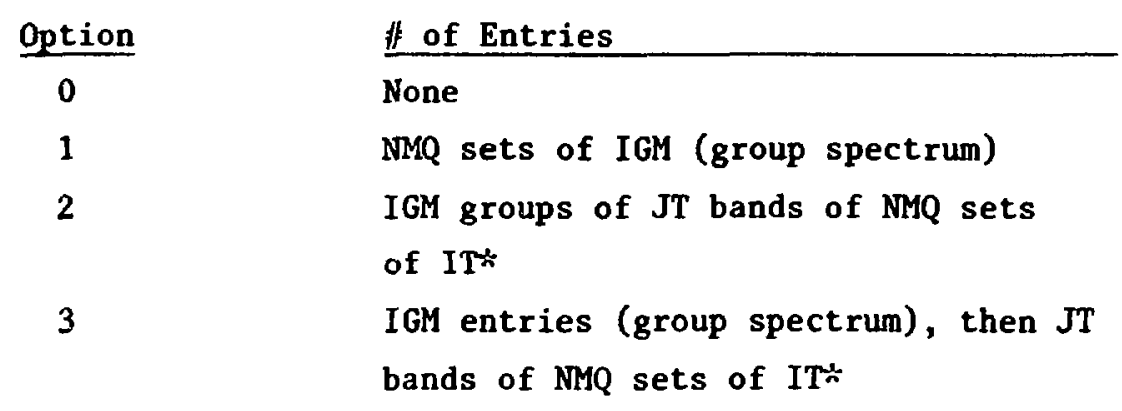

Boundary source input, read in NBSG groups of the boundary source in the following order on a $S(E)$ format. Enter only if NBSG>0.

Array

BL (LMBL , JT , 4*MNPQ)

BLI ( IBLI , JLT , 4\%MNPQ)

$\mathrm{BR}(\mathrm{LMBR}, \mathrm{JT}, 4 \div \mathrm{MNPQ})$
Comments

$L M B L=1$, for $I B L=-3$; $L M B L=2$ for $I B L=3$. Enter only if $\operatorname{IABS}(\mathrm{IBL})=3$.

$\mathrm{JLT}=\mathrm{JLIT}-\mathrm{JLIB}+1 . \quad$ Enter only if IBLI>0.

$L M B R=1$, for $I B R=-3$; $L M B R=2$ for $I B R=3$. Enter only if $\operatorname{IABS}$ (IBR) $=3$. 
BRI (IBRI , JRT , 4*MNPQ)

$\mathrm{BT}_{\text {in }}$ (MNPQ, LMBT , NDNT)

BT ${ }_{\text {out }}$ (MNPQ, LMBT, NDNT)

BTI (2*MNPQ, IBTI, NBTI)

$\mathrm{BB}_{\text {in }}$ (MNPQ, LMBB , NUPB)

${ }^{B B}{ }_{\text {out }}$ (MNPQ, LMBB , NUPB)

BBI ( $2 * M N P Q, I B B I, N B B I)$

Block Name

\& Dimension

IDCS (ITMAX)

CHI (IGP)

$S(E)$

VEL(IGP)

S(E)

FSG(IGM)
$J R T=J R I T-J R I B+1 . \quad$ Enter only if IBRI $>0$.

LMBT $=1$, for $I B T=-3$ and $L M B T=2$ for $I B T=3$.

Enter only if IABS(IBT) $=3$

Enter only if IBTI $>0$.

$\angle M B B=1$, for $I B B=-3$; $L M B B=2$, for $I B B=3$.

Enter only if $\operatorname{IABS}(\mathrm{IBB}) \div 3$.

Enter only if $I B B I>0$.
\# of

Format Entries

S(I) IT*

\section{Comments}

Cross section material identifica-

tion numbers. These numbers assign a

cross-section block to each triangle.

If these numbers are negative, an

anisotopic scattering source is

calculated in that triangle, but the

numbers need not be negative when

ISCT $>0$. JT bands of IT* are entered, for MESHOPT=0, IT*=ITZ(J) and for

MESHOPT $=1$, IT $*=\mathrm{IT}(\mathrm{J})$

Fission fractions. Fraction

of fission yield emerging in

each group

Group speeds. Used only in

time-absorption calculations.

Fictitious-source group con-

stants (called $\beta$ in Sec. III.E).

These constants are limited to

the interval $0 \leq \beta_{\mathrm{g}} \leq 1$. Do

not enter if IFS $=0$ 


\begin{tabular}{|c|c|c|c|}
\hline MIXNUM(MS) & $S(I)$ & MS & $\begin{array}{l}\text { Numbers identifying cross sec- } \\
\text { tion block being mixed. See } \\
\text { Sec. III.U. Do not enter } \\
\text { if } M S=0 \text {. }\end{array}$ \\
\hline MIXCOM(MS) & $S(I)$ & MS & $\begin{array}{l}\text { Numbers controlling cross } \\
\text { section-mixture process. See } \\
\text { Sec. III.U. Do not enter } \\
\text { if MS }=0 .\end{array}$ \\
\hline MIXDEN (MS) & $S(E)$ & MS & $\begin{array}{l}\text { Mixture densities. See } \\
\text { Sec. III.U. Do not enter } \\
\text { if MS }=0 .\end{array}$ \\
\hline $\begin{array}{l}\text { BHT (1) or } \\
\text { BHT (ITMAX) }\end{array}$ & $S(E)$ & $\begin{array}{l}1 \text { or } \\
\text { IT }\end{array}$ & $\begin{array}{l}\text { Buckling heights (in cm if } \\
\text { cross sections are in barns). } \\
\text { Used to simulate z-dimension } \\
\text { of an } x-y \text { geometry problem by } \\
\text { adding an absorption given by } \\
\begin{array}{c}\sigma_{a, B H T}= \\
\quad \frac{\sigma}{3}\left[\pi / \text { BHT }^{2} \sigma_{t}+1.4209\right]^{2}\end{array}\end{array}$ \\
\hline & & & $\begin{array}{l}\text { Here } 1.4209 \text { is twice the Milne } \\
\text { problem extrapolation distance, } \\
\text { and } \sigma_{t} \text { is the total cross sec- } \\
\text { tion. For ISDB }=0 \text {, a single, } \\
\text { whole-system buckling height } \\
\text { is entered, for ISDB }=1 \text {, JT } \\
\text { bands of IT* are entered } \\
\text { for MESHOPT }=0, I T^{*}=\operatorname{ITZ}(\mathrm{J}) \text {, } \\
\text { and for MESHOPT }=1, \operatorname{IT} *=\operatorname{IT}(\mathrm{J})\end{array}$ \\
\hline
\end{tabular}

E. Edit Input

The edit input, entered only if IEDOPT $=1$, consists of control integers entered on cards indicated by EDIT 1, 2, or 3; and the remaining edit input is entered on the LOAD format discussed above. The zone-edit control integers and the zone-edit arrays are read first for all NZEDS edits, then the point-edit control integers and point-edit arrays are read in for all NPEDS edits. 
EDIT CONTROL INTEGERS (2I6)

1 NZEDS Number of zone edits

2 NPEDS Number of point edits

ZONE EDIT CONTROL INTEGERS, ENTER ONLY IF NZEDS $>0$ (4I6)--.-.-EDIT 2

$1 \quad$ NZ Total number of zones

2 NCA Number of constituent activities calculated

3 NMA Number of microscopic activities calculated

4 NORMZ Zone identification number for normalization of power density. If NORMZ $=0$, whole system normalization is performed.

IDCA(NCA)

$\operatorname{IDMA}(\mathrm{MMA})$

NEDZ (ITMAX)
$S(I)$

S(I)

S(I)
NCA cross section material identification numbers for constituent activities. Enter only if NCA>0 NMA cross section material identification numbers for microscopic activities. Enter only if NMA>0.

IT* zone identification numbers. These numbers assign a zone number to each triangle. Enter JT bands of IT* where for MESHOPT $=0 \quad \operatorname{IT} *=\operatorname{ITZ}(\mathrm{J})$, for MESHOPT=1 IT* $* I T(J)$.

POINT-EDIT CONTROL INTEGERS, ENTER ONLY IF NPEDS $>0,(316)$ -EDIT 3

1 NTPE $0 / 1$ if equal to zero all triangles are included in pointedit, if greater than zero, the point edit is entered on a per band basis

2 NJPE Number of bands included in the point edit

3 NPMA Number of microscopic activities calculated in the point edit

IDMA(NPMA)

JDC (NJPE)
S(I) NPMA

$\mathrm{S}(\mathrm{I}) \quad$ NJPE
Cross section material identification numbers for microscopic activities. Enter only if NPMA>0.

Band identification numbers of the bands that have triangles included in the point 
edit. No entries are made if NTPE=0. Point edit indication numbers. Enter NJPE bands of IT $*$,

for MESHOPT $=0, I T *=\operatorname{ITZ}(\mathrm{J})$,

for MESHOPT $=1$, IT $\div$ IT $(J)$.

If triangle $I$ is not included in the point-edit, $\operatorname{NEDP}(I)=0$ and if it is included in the edit, $\operatorname{NEDP}(I)=1$. No entries when NTPE $=0$.

F. Plot Edit Input

The 3-D plot edit input, entered only if IEDPLT $=1$, consists of control integers entered on cards indicated by PLOT EDIT 1, 2, 3, or 4; and the remaining plot edit input is entered on the LOAD format discussed above. The material plot control integers and the material plot arrays are read in for all NMTPLT material plots, then the flux-plot control integers and flux-plot arrays are read in for all NFXPLT flux plots, and then the response function plot integers and response function plot arrays are read in for all NRFPLT response function plots. In entering the BOUND array XMIN and XMAX refer to the minimum and maximum values on the $\mathrm{x}$ or $\mathrm{r}$ axis and YMIN and YMAX refer to the same values for the $\mathrm{y}$ or $\mathrm{z}$ axis. Entries of zero for both XMIN and XMAX or YMIN and YMAX result in the actual problem domain being used in the $\mathrm{x}$ or $\mathrm{y}$ direction.

PLOT EDIT CONTROL INTEGERS (5I6) 1

1 NMTPLT Number of material 3-D plots

2 NFXPLT Number of flux 3-D plots

3 NRFPLT Number of response function 3-D plots

4 NX Number of evenly spaced plotting divisions along the $\mathrm{x}-$ (or $\left.\mathrm{r}^{-}\right)$) axis.

$5 \quad$ NY Number of evenly spaced plotting divisions along the $\mathrm{y}-\left(\right.$ or $\left.\mathrm{z}^{-}\right)$axis.

(The 3-D plots are made on a $\mathrm{NX}$ by $\mathrm{NY}$ rectangular grid.)

MATERIAL PLOT EDIT CONTROL INTEGERS, ENTER ONLY IF NMTPLT>0,(1I6) PLOT EDIT 2

1 NMT Number of materials to be included in material plot 
IDMA(NMT) S(I) NMT cross section material identification numbers.

BOUND(4) S(E) Coordinate values defining the rectangular a reas to be plotted. Entered in the following order: XMIN, XMAX, YMIN, and YMAX.

FLUX PLOT EDIT CONTROL INTEGERS, ENTER ONLY IF NFXPLT>0, (116) ------ PLOT EDIT 3

1 NFXG Number of flux groups to be plotted.

IGPLT(NFXG) S(I) NFXG identification numbers of the flux groups to be plotted. No entry required if NFXG $\geq$ IGM. An entry of IGM+l yields the group summed flux.

BOUND(4) $\quad S(E)$ Coordinate values defining the rectangular areas to be plotted. Entered in the following order: XMIN, XMAX, YMIN, and YMAX.

RESPONSE FUNCTION PLOT CONTROL INTEGERS, ENTER ONLY IF NRFPLT>0, (3I6) PLOT EDIT 4

1 NRFG Number of flux groups to be used in the response function edit.

2 NMT Number of materials to be used in the response function edit.

3 IPP Cross section table position to be used in the response function edit.

IGPLT(NRFG) S(I) NRFG identification numbers of the flux groups to be used in the response function edit. No entry if $N R F C=I G M$.

IDMA(NMT) S(I) NMT cross section material identification numbers, specifying the materials to be used in the response function edit. 
BOUND (4)

$S(E)$

Corrdinate values defining the rectangular area to be plotted. Entered in the following order: XMIN, XMAX, YMIN, and YMAX.

\section{DETAILED INPUT DESCPIPTION}

This section is a supplement to the brief explanations of the input variables specified in the preceding section. The description format follows exactly the order in which the input is read into the code.

To illustrate the use of the LOAD format, discussed at the beginning of Sec. II, Fig. 4 gives five examples of the special format. Table II is a repeat, provided for convenience. Figure 4 illustrates the following:

1 - Zero is repeated 47 times.

2 - Zero is repeated 470 times.

3 - Four interpolants are inserted between 0.0 and 5.0

giving six data numbers: $0.0,1.0,2.0,3.0,4.0,5.0$.

4 - Four interpolants are inserted betwen 0.0 and 5.0,

two between 5.0 and 7.0 , and 7.0 is repeated 10 times.

5 - After reading 0 and 4 we skip to the next card and read 7

TABLE II

OPTIONS FOR SPECIAL LOAD FORMAT

Value of I1

0 or blank

1

2

3

4

5

Nature of Option

No action.

Repeat data word in 9 field number of times indicated in 12 field.

Place number of linear interpolants indicated in 12 field between data word in 9 field and data word in next 9 field. Not allowed for integers.

Terminate reading of data block. A 3 must follow

last data word of all blocks.

Fill remainder of block with data word in 9 field.

This operation must be followed by a terminate ( 3 ).

Repeat data word in 9 field 10 times the value in the

12 field.

Skip to the next data card. 


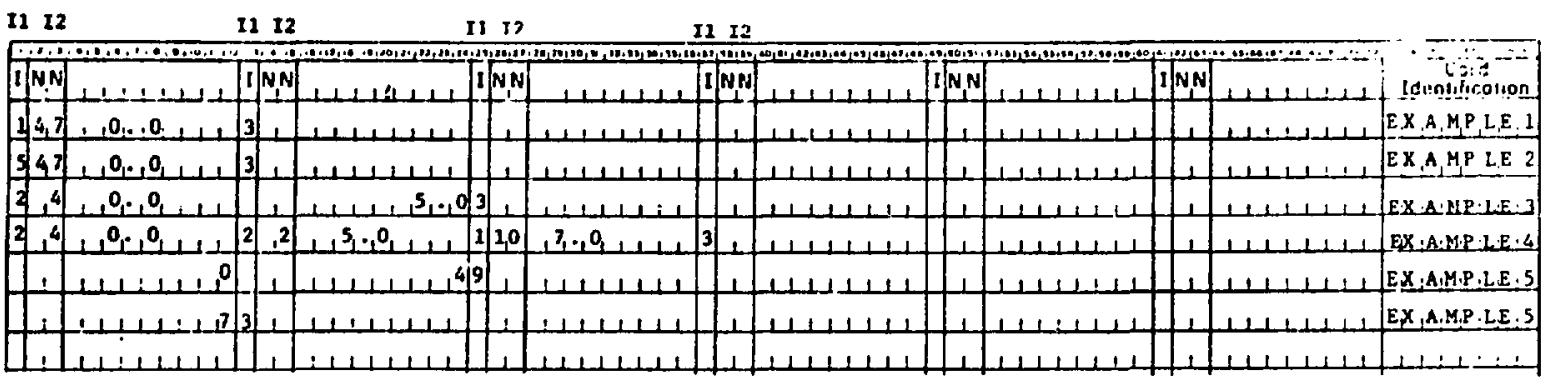

Fig. 4. Illustration of format read by LOAD

\section{A. Card One Entry One ITH}

$0 / 1$ (direct/adjoint) calculations. Direct refers to the solution of the transport equation in the standard sense.

TRIDENT-CTR solves the adjoint transport equation by transposing the matrices of scattering coefficients and inverting the group order of the problem. The solution of the resulting problem in direction $\Omega$ is then identified with the solution of the adjoint equation in direction $-\Omega$.

The inversion of the group order is made because the transposition of the scattering matrices usually converts a downscattering problem to an upscattering problem. Because of the inversion, the user must:

(a) enter any inhomogeneous sources, including boundary fluxes, in inverse group order,

(b) enter any flux guess in inverse group order, and

(c) remember that any output is in inverse group order, 
i.e., that groups labeled $1,2, \ldots$, are really groups IGM,

IGM - 1, etc.

Similarly, the output flux from an adjoint problem must be inverted before insertion in a direct problem. On the other hand, an output flux from one adjoint problem is in the proper group order for use in another adjoint problein. The group order of the group speeds and the fission spectrum is inverted by the program. Note that the fictitious source option may not be used in the adjoint mode.

B. Card One, Entry Two ISCT

$0 / \mathrm{N}$ (isotropic/N ${ }^{\text {th }}$ order anisotropic) scattering calculations.

TRIDENT-CTR uses the standard approximation for anisotropic scattering where the scattering transfer probability is assumed to be represented by a finite Legendre polynomial expansion. The approximation results in the angular flux being expanded in a set of associated Legeridre polynomials. The derivation of this approximation is contained in several two-dimensional transport code manuals $1,2,3,4$. A scattering approximation of ISCT requires ISCT+1 cross-section decks for each anisotropic material and $($ ISCT +1$) *(I S C T+2) / 2$ angular flux moments. ISCT should always be at least one less than the $S_{N}$ order.

C. Card One, Entry Three IGEOM

$1 / 2(x-y / r-z)$ geometry option.

TRIDENT-CTR solves both the $x-y$ and $r-z$ formulation of the neutral particle transport equation. Special restrictions are:

(a) TRIDENT-CTR requires that for $r-z$ geometry, the quadrature set be symmetric with respect to the $\eta$ axis regardless of the boundary conditions, and

(b) the special boundary condition does not apply and cannot be used in $r=2$ geometry.

The $x-y S_{N}$ equations are much simpler than the $r-z$ equations and require significantly less time to solve. If the problem can be formulated in $x-y$ geometry, it is more efficient to do so.

D. Card One, Entry Four IGM

Number of energy groups.

In TRIDENT-CTR the energy variable is approximpted with the standard multigroup approximation. The energy domain of interest is assumed to be partitioned into IGM intervals of width $\Delta \mathrm{E}_{g}, g=1,2, \ldots$, IGM. By convention, increasing $g$ represents decreasing energy. If we integrate the flux for group $g$, 


$$
\psi_{\mathrm{g}}=\int_{\Delta_{\mathrm{E}}} \psi \mathrm{dE}
$$

it is no longer a distribution in energy, but is the total number of particles in the energy interval. For this reason, when group structures are changed, the effect on results must be evaluated by comparing $\psi_{g} / \Delta E_{g}$. Energy integrals in TRIDENT-CTR are evaluated by simple sums.

The cross sections subscripted with $g$ are average, e.g.,

$$
\sigma_{t g}=\int_{\Delta E} \sigma_{t} \psi d E / \int_{\Delta E} \psi d E,
$$

but, of course $\psi$ is not known and must be approximated by some means. If the angular dependence of $\psi$ is nonseparable, then $\sigma_{t g}$ will depend on angle. No provision for such dependence is made in TRIDENT-CTR. Recipes for taking this dependence into account, as well as for improving the averages $\sigma_{s \ell h \rightarrow g_{9}}$ when scattering is severely anisotropic, are given by Bell, Hansen, and Sandmeier.

E. Card One Entry Five IFS

$0 / 1\left(\mathrm{~S}_{\mathrm{N}} /\right.$ spherical harmonics) fictitious source option. (Ray-effect fixup.)

The discrete ordinates approximation results in distorted flux distributions for a certain class of problems. The physical problems in question are those with more-or-less physically isolated sources and with low scattering ratios. The flux plots have so-called ray effects ${ }^{7}$ which are anomalous ripples that may be confused with spatial flux variation due to physical effects. The distortion is due to the fact that a finite number of directions is used resulting, for some problems, in subregions which are not penetrated by rays originating at sources. Increasing the number of angular directions does mitigate the effect since more of the problem area is penetrated by rays originating in scattering, fission, or distributed sources. The ripples, however, are often remarkably persistent, even when a large number of directions is used. 10

In order to eliminate the ray effect in problems where it is likely to appear, TRIDENT-CTR includes the option to append a fictitious source to the discrete ordinates equations defined so that spherical harmonics ( $x-y$ geometry) or spherical harmonics-like ( $r-z$ geometry) solutions result. The spherical harmonics equations are rotationally invariant and do not demonstrate the ray effect. 10 
The fictitious source that is appended to the discrete ordinates equation in TRIDENT-CTR is the Miller-Reed ${ }^{6,8}$ fictitious source. When the source is added, it is multiplied by a group-dependent constant $\left(\beta_{g}\right) . \beta_{g}$ is restricted such that $0 \leq \beta g \leq 1$. If $\beta_{g}=0$, the standard discrete ordinates solution results. For $\beta=$ 1.0 , a spherical harmonics solution of order N-1 results. For intermediate values of $\beta_{g}$, the solution is a linear combination of discrete ordinates and spherical harmonics solution results.

As will be pointed out several times, the addition of the fictitious source to the discrete ordinates equations eliminates the ray effect but results in two significant penalties. First, the discrete ordinates equations are solved iteratively and when the ray effect is severe (the fictitious source is large) the iteration is slow to converge. By selecting a $\beta<1(\mathrm{e} . \mathrm{g}, \beta=0.9)$, the ray effect may be reduced and not completely eliminated with less of a run-time penalty. 6,8 Second, the spherical harmonics solutions are less accurate than discrete ordinates solutions in terms of spatial integral parameters (e.g., total absorption). Thus, if one desires ray-effect mitigation with small loss in integral parameter accuracy, $\beta<1$ may be used. Note further that since $\beta$ is group dependent the user can mitigate the ray effect only in problem energy groups and then convergence penalties are only paid for those groups for which $\beta>0$. Although for most problems, the fictitious source is small and does not significantly affect convergence, the user should use the fictitions source option only when necessary due to the large computer storage required.

Restrictions on the use of the fictitious source option are:

(a) the fictitious source option may not be used for adjoint calculations, and

(b) the built-in $\mathrm{EQ}_{\mathrm{N}}$ quadrature set must be used.

F. Card One, Entry Six IEVT

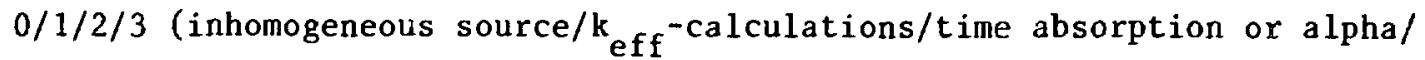
concentration search).

TRIDENT-CTR will solve both the inhomogeneous (source) and the homogeneous ( $k_{\text {eff }}$ ) form of the transport equations. When the homogeneous solution is sought, the term representing the source of neutrons due to fission is divided by $k_{e f f}$. It is possible in TRIDENT-CTR to adjust nuclide concentrations or the value of the time absorption to achieve a desired value of $k_{\text {eff }}$. This value is taken to be unity (criticality) unless the parametric eigenvalue trigger (IPVT) is set to unity. In this case, the parametric value of $k_{e f f}$ is entered as an input number. 
If IPVT=2, a $1 / v$ absorber of value PV is added to the problem in each space cell.

The modification of cross section concentrations takes place when the eigenvalue type indicator (IEVT) is a 3. If IEVT is 2 (time absorption computation), the value $\mathrm{EV} / \mathrm{v}_{\mathrm{g}}$ is added to the absorption and total cross sections in each group. Here $v_{g}$ is the speed associated with energy group $g$.

Regardless of the parameter being adjusted, the search is executed by performing a sequence of $k_{\text {eff }}$ calculotions, each for a diffexent value of the parameter being treated as the eigenvalue. Each of the successive $k_{e f f}$ calculations is accelerated by rebalance, but the search for the desired value of $k_{\text {eff }}$ is conducted by subroutine NEWPAR. Regardless of the nature of the problem, the search is for a value of the parameter which makes the value of $\lambda$ defined as

$$
\lambda^{(r)}=\frac{\text { Fission Source } e^{r}+\text { Inhomogeneous Source }}{\text { Fission Source }}
$$

In the following description of NEWPAR, it is helpful to refer to Fig. 5 in which the deviation of $\lambda$ from unity is plotted against outer iteration number. For the initial system NEWPAR continues outer iteration until two successive values of $\lambda$ differ by less than EPSO. For subsequent sequences of $\lambda$ values a different convergence precision, XLAX, is used. After the first converged $\lambda$ sequence is obtained, the initial value of the eigenvalue (EV) is altered by EVM, an input value. If $\lambda>1$ (multiplying system), the new eigenvalue is equal to EV + EM; if $\lambda<1$ (decaying system), the new value is EV - EVM. These alterations correspond to the addition or the subtraction of an absorption (e.g., as in a timeabsorptiun search or a poison-concentration search).

Basically, after two values of $k_{\text {eff }}(\lambda)$ are obtained for two different system configurations, subroutine NEWPAR attempts to fit a curve through the most recent values to extrapolate or interpolate to a value of unity. Depending on the amount of information available and the size of $1-\lambda$, this fit proceeds in different ways. A parabolic fit cannot be made until three converged values of $\lambda$ are available, and is not attempted unless $1-\lambda$ is greater than an input-search lower limit (XLAL) and less than an input-search upper limit (XLAH). If a parabolic fit is tried and the roots are imaginary, a straight-line fit is used. If the roots are not imaginary, the closest root is used as the new value of EV. Once a bracket 
is obtained (change of sign of $\lambda-1$ ), the fit procedure is not allowed to move outside the region of the bracket. Should a parabolic fit select an eigenvalue outside the bracket region, this value is rejected and the new value is taken to be one-half the sum of the previous value and the value previous. to that.

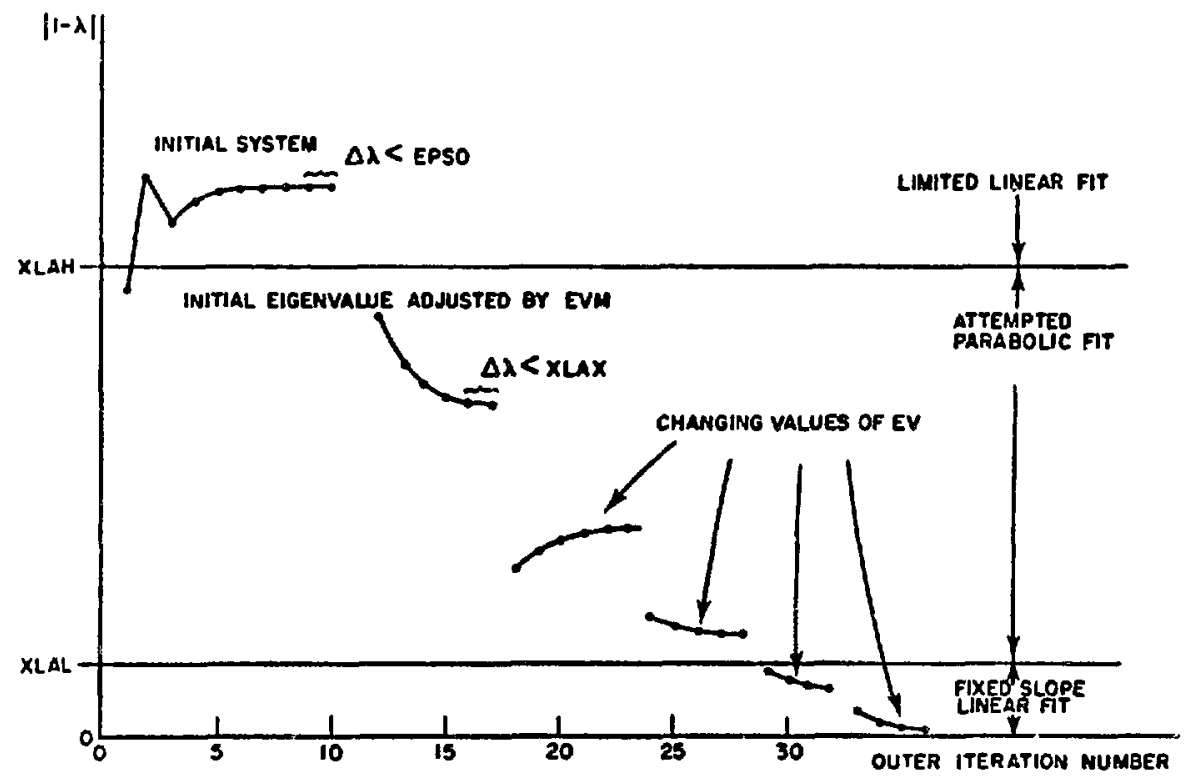

Fig. 5. Variation of $\lambda$ during a hypothetical eigenvalue search. 
Whenever the parabolic fit is not used, a linear fit is used and the new eigenvalue is computed from

$$
(E V)_{\text {new }}=(E V)_{\text {old }}+P O D * E Q *(1-\lambda) \text {, }
$$

where POD is an input "parameter oscillation damper" which may be used to restrict the amount of char ${ }^{-}$in the eigenvalue. EQ is a measure of the slope $o_{\perp}$ the curve. When $1-i ; \mathrm{XLAH},(1-\lambda)$ is replaced by XLAH (with the correct sign) to prevent too large a change in EV. After $1-\lambda<\mathrm{XLAL}$, the value of EQ is fixed and kept constant until convergence to prevent numerical difficulty in the approximation of the derivative when $\lambda$ is close to unity.

Because parametric search problems represent sequences of $k_{\text {eff }}$ calculations, it behooves the user to study the use of subroutine NEWPAR in order to optimize his calculations. It also behooves the user to pose soluble problems. That is, there are many problems, especially concentration searches, for which solutions are not possible, and discovering this by trial and error is the hard way. Ideally, the user will have some estimate of the critical parameter available from a lower order computation.

Convergence in time-absorption calculations is typically one sided. If EV is negative, then there is a possibility that the corrected total cross section will become negative. If this happens, the automatic search procedure may fail dramatically. For this reason POD $=0.5$ or less is frequently used in such searches.

TRIDENT-CTR does not have the fine-mesh rebalance acceleration option of TRIDENT. Also because of the increased flexibility of the mesh specification, TRIDENT-CTR's solution equations are more time consuming than TRIDENT's. Because of these two factors, eigenvalue-type problems that can be set up on TRIDENT will run faster than on TRIDENT-CTR.

G. Card One, Entry Seven ISTART

$(7 / 6 / 5 / 3 / 2 / 1 / 0 / 1 / 2 / 3 / 5 / 6 / 7)$ input flux guess and starting options.

If ISCT is the order of scattering, then there are $\mathrm{NM}=(\mathrm{ISCT}+1) *(\mathrm{ISCT}+2) / 2$ spherical harmonic components of the angular flux. The flux is expanded with a finite spherical harmonics expansion. The components are ordered as in Table III. Options for entering these components are selected by the input value of the integer ISTART. A negative value for ISTART indicates that only the angle-integrated or scalar flux is to be read. 
For ISTART $=5,6$, or 7 , a problem restart is made from previously generated binary flux files. The files, RTFLUX, ATFLUX, and RAFLUX, specify within themselves which flux groups they contain and what spatial mesh was used to generate the flux. If the spatial mesh within the flux file does not match the problem mesh, the problem is aborted. It may take one, two, or all three files to contain previously calculated fluxes depending upon the problem size. For example in a 42-group problem, RTFLUX could contain groups 1-16, ATFLUX groups 17-32, and RAFLUX groups 33-42.

For ISTART $=1,2$, and 3 , the flux is entered as a starting guess on cards. If MSHOPT (automatic mesh generator option, Sec. III.P) $=0$ the flux is entered on a per band basis for every zone. For MSHOPT=1 the flux is entered for every point in the problem. (three points per triangle, at the vertices.)

TABLE III

ORDERING OF ANISOTROPIC FLUX AND DISTRIBUTED

SOURCE COMPONENTS

Component Number

1

2

3

4

5

6

7

8

9

10

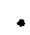

etc. k

0

0

1

0

1

2

0

1

2

3 
The various options are broken down as follows:

\begin{tabular}{|c|c|}
\hline$\underline{\text { ISTART }}$ & Option \\
\hline$-7,-6,-5,-3,-2,-1$ & Same as $+7,+6,+5,+3,+2,+1$, isotropic component only. \\
\hline 0 & $\begin{array}{l}\text { No flux guess entered, a flux of zero and a fission } \\
\text { guess of unity in every cell is supplied. }\end{array}$ \\
\hline 1 & $\begin{array}{l}\text { Enter an energy spectrum }\left(\mathrm{GR}_{\mathrm{g} \ell}, \mathrm{g}=1 \text {, IGM }\right) \text { for each } \\
\text { anisotropic component } \ell \text {. The flux guess is then } \\
\text { formed as } \text { FLUX }_{\mathrm{g} \ell \mathrm{ki}}=\mathrm{GR}_{\mathrm{g} \ell} ; \mathrm{k}=1,3 ; \mathrm{i}=1, \mathrm{NTC} \text { for each } \\
\ell \text { and } \mathrm{g} \text {. }\end{array}$ \\
\hline 2 & $\begin{array}{l}\text { Enter the entire flux array as IGM groups of JT } \\
\text { bands of NM sets of IT* (see below). }\end{array}$ \\
\hline 3 & $\begin{array}{l}\text { Enter first any energy spectrum and then a spatial } \\
\text { shape as JT bands of NM sets of IT\%. }\end{array}$ \\
\hline 5 & Read in fluxes from binary flux file RTFLUX. \\
\hline 6 & Read in fluxes from binary files RTFLUX and ATFLUX. \\
\hline 7 & $\begin{array}{l}\text { Read in fluxes from binary files RTFLUX, ATFLUX, } \\
\text { and RAFLUX. }\end{array}$ \\
\hline
\end{tabular}

$I T^{*}=\operatorname{ITZ}(\mathrm{J})$ (number of zones on band $\mathrm{J}$ ) for MSHOPT $=0$

$I T *=3 * I T(J)$ (number of triangles on band $\mathrm{J}$ ) for MSHOPT $=1$

H. Card One, Entry Eight ISTOP

$-2 /-1 / 0 / N$ (After 1 outer/after input is read in/none/after N-groups) stop option.

TRIDENT-CTR's normal mode of operation is to perform at least two outer iterations. When solving a inhomogeneous source problem with no fissioning or upscattering and when an angular flux file is not desired, and ISTOP value of -2 will terminate the problem after one inner. The use of this option can save the time cost of going through an extra outer when not needed or desired.

An ISTOP value of -1 will read in the input, skip the coiaputational portion of the code and perform all output portions of the code. The user may use this option to ensure his input is correct before performing the actual computations.

When used with the ISTART option, a previously calculated flux file may be read in and edited with the edit portion of the code.

Likewise with ISTART set to a positive integer, the problem may be terminated 
normally after ISTART groups have been calculated. A flux file will be created for later restart and parameters of interest may be examined (through the edits) before continuing the calculation.

These ISTART options, other than zero, were intended for use primarily with no upscatter, source problems. Caution must be exercised when using these options with other than this type of problen.

I. Card One, Entry Nine IQOPT

$0 / 1 / 2 / 3$ inhomogeneous source input options.

The TRIDENT-CTR user may specify an anisotropic distributed source. The inhomogeneous distributed source must be represtated by the finite spherical-harmonics expansion

$$
Q(r, \mu, \eta)=\sum_{\ell=0}^{\operatorname{IQAN}}(2 \ell+1) \sum_{k=0}^{\ell} R_{\ell}^{k}(\mu, \phi) Q_{\ell}^{k}(r)
$$

in which IQAN is an input number designating the order of anisotropy of the source and $R_{\ell}^{k}$ are the spherical-harmonics polynomials. In these terms,

$$
Q_{\ell g}^{k}=\frac{1}{4 \pi} \int_{-1}^{1} d \mu \int_{0}^{2 \pi} d \phi Q_{g}(r, \mu, \eta) R_{\ell}^{k}(\mu, \phi)
$$

We have written the integral in this equation over the entire range of $\phi$ and used a $4 \pi$ normalization of the integral, but it should be clear that $Q_{\mathrm{g}}$ must be symmetric in $\phi\left[Q_{g}(\phi)=Q_{g}(-\phi)\right]$. (where $\phi=\cos ^{-1}\left(\frac{n}{\sqrt{1-\mu^{2}}}\right)$ )

In addition to specifying IQAN, the user must enter $N M Q=[(I Q A N+1)(I Q A N+2)] / 2$ components of $Q_{g}$ multiplied by $(2 \ell+1)$; that is, the user must enter

$$
\begin{aligned}
(2 \ell+1) Q_{\ell_{g}}^{k} k & =0,1, \ldots, \ell \\
\ell & =0,1, \ldots, \text { IQAN }
\end{aligned}
$$


in the order shown in Table 3 . When using the anisotropic-distributed-source option, the order of anisotropic scattering, ISCT, must be at least as large as IQAN so that the requisite number of polynomials $R_{\ell}^{k}$ is computed.

The IQOPT parameter provides the TRIDENT-CTR user with four options for entering the distributed source. These options are:

$\underline{\text { IQOPT }}$

0

1

2

3.

J. Card One, Entry T'en NQG

Number of inhomogeneous source groups.

In fission transport codes there have traditionally been as many source groups as flux energy groups. For fission type shielding problems the source is distributed throughout the energy range. For fusion problems this is not true. and the source resides only in one or two of the high-energy groups. For these problems it would be a waste of storage to store a source that was zero throughout most of the energy range. Hence in TRIDENT-CTR the number of source-energy groups is specified separate from the number of flux energy groups. The energy endpoints of all the source-energy groups must correspond to flux-energy groups.

K. Card One, Entry Eleven NBSG

Number of boundary source groups.

The arguments for limiting the inhomogeneous source groups also applies to the number of boundary source groups.

L. Card One, Entry Twelve JSTART

Group restart number.

JSTART can be used with the flux restarts to limit the number of iterations made in previously calculated groups. If a partial flux dump had previously been made and the inner iterations for the groups calculated had been terminated by it- 
eration count, then by setting JSTART, calculations in these groups on the restart can be limited to 1 iteration per group. For example in a 30 group calculation, a restart is made with a binary flux file containing the first 15 groups. By setting JSTART to 16 , the code will make one inner iteration per group until group 16 is reached. At that point the input iteration limit will be used. The one inner iteration is required to set the codes balance tables.

M. Card Two, General Comments on Boundary Control Integers Specified on Entries One through Eight

The equations and methods of TRIDENT-CTR determine the angular flux within a triangle provided the incoming flux on triangle boundaries is known. This incoming flux may be known from prior calculation in adjacent cells. If an incoming triangle boundary is a system boundary, then the incoming flux must be determined from the appropriate boundary condition. In TRIDENT-CTR, one of the boundary conditions described below must be applied to the top, bottom, and each of the two sides of a system. The top and bottom ioundaries are defined to be those parts of the system perimeter coinciding with the top and bottom horizontal lines (node lines), respectively. Note that the top and bottom boundaries are always flat, but side boundaries may approximate some curved surface. It is possible for the top or bottom boundary or both to fail to exist. This happens, for example, if there is a single up-pointing triangle on the top band. In these cases the boundary conditions are meaningless and are not used.

In addition TRIDENT-CTR allows definition of a top, bottom, left, and right internal boundary for which a boundary source may be specified.

\section{Vacuum Boundary Condition}

The value of the angular flux on the boundry is set equal to zero for all incoming directions. Unlike some two-dimensional transport codes, (e.g., TWOTRAN), TRIDENT-CTR allows a vacuum boundary condition on the left side in $r-z$ geometry. This feature is useful for modeling some toroidal configurations.

\section{Reflective Boundary Condition}

Reflective boundary condiiions may be applied to each of the four sides in any combination. A reflective boundary must be parallel with one of the coordinate axes, and the quadrature mist possess certain symmetries. If either of the side boundaries is reflective, the quadrature must be symmetric about the line $\mu$ $=0$ in the $\mu-\eta$ plane, whereas if either the top or bottom boundary is reflective, the quadrature must be symmetric about $\eta=0$. These requirements are necessary 
to guarantee that all reflections of directions are contained in the quadrature set. The built-in $\mathrm{EQ}_{\mathrm{N}}$ quadrature set provides these symmetries for any boundary parallel to one of the coordinate axes.

For a reflective boundary condition, the value of the incoming flux on the boundary is set equal to the value of the outgoing flux at the same position in the direction corresponding to specular reflection. At the right-hand boundary, for example, we set '

$$
\psi_{\text {incoming }}(-\mu, \eta)=\psi_{\text {outgoing }}(\mu, \eta), \mu>0
$$

\section{Special Boundary Condition}

A special boundary condition, useful for hexagonal cell calculations, is provided in TRIDENT-CTR. This boundary condition can be used on the right-hand boundary, in $x-y$ geometry only, and must be used in conjunction with reflective boundary conditions on the other three sides. When a special boundary condition is used, the band widths must be symmetric about a horizontal line through the middle of the system. There may be an even or odd number of bands; if there is an odd number of bands, the centermost band is unpaired and may be any size.

Figure 6 illustrates the use of the special boundary to represent a hexagonal cell. This boundary condition is implemented in the following way. We assume there are a total of JT bands. The incoming flux on the $J^{\text {th }}$ band in the direction $(-\mu,-\eta)$ is set equal to the outgoing flux on band JT $+1-J$ in the direction $(\mu, \eta)$. Furthermore, the spatial variation of the flux within the band is reversed. Hence the special boundary condition is really a $180^{\circ}$ reflection combined with a spatial transposition about the horizontal centerline of the system, and is sometimes referred to as a "180" rotational" boundary condition.

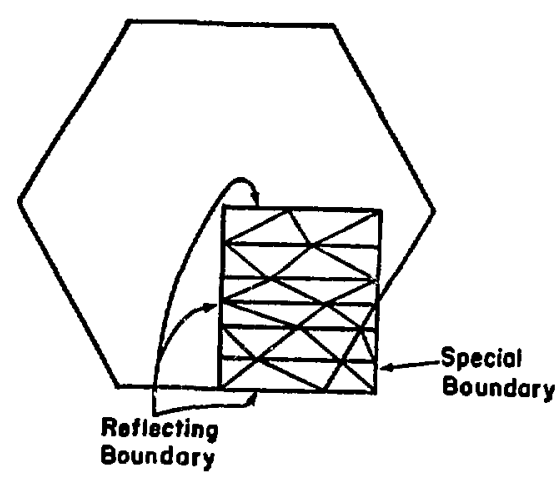

Fig. 6. Use of the special boundary condition to represent a hexagonal cell. 


\section{White Boundary Condition}

The values of the incoming boundary fluxes are all set equal to the same value. This value is the average of the outgoing fluxes such that the net flow through the boundary is zero. For example, for a right boundary this value is given by

$$
\psi_{\text {incoming }}=\frac{\sum_{m=1}^{M M} w_{m} \mu_{m} \Psi\left(\mu_{m} \eta_{m}\right) \text { outgoing }}{\sum_{m=1}^{M M} m^{w} w_{m} \quad \text { outgoing }}
$$

\section{Boundary Sources}

Boundary sources may be specified for any or all of the four external boundaries (left, right, bottom, and top), and four internal boundaries (left internal, right internal, bottom internal, and top internal). Unlike the inhomogeneous source which is specified in terms of a spherical harmonics expansion, the boundary source is specified as an incoming angular flux in the appropriate quadrature directions. It is not necessary when using this boundary condition that the left or right sides be parallel to the $y$ or $z$ coordinate axis, and an incoming direction at one point on the boundary may be an outgoing direction at other points. Because it is therefore impossible to guarantee which directions are incoming directions, the user must enter a value for all directions at every point along a left or right side source boundary. TRIDENT-CTR then selects and uses only the values corresponding to incoming directions.

The shaped source boundary condition means that the boundary source is specified at each triangle vertex along the boundary. The flat source boundary condition means the boundary source is specified as one value for each triangle along the boundary. Figure 7 illustrates the various possible boundary sources, with the arrows indicating the sense of incoming for each boundary. As an example, the left boundary source enters the problem domain from left to right while the left internal boundary source enters by appearing at an internal boundary moving from right to left.

Boundary sources represent a source of particles and are treated as sources by the code. 
N. Card Two, Entry Nine - JT

Number of bands of triangles.

The triangular mesh used by TRIDENT-CTR is not a general triangular mesh. The horizontal banded structure requires that all triangle vertices must lie on horizontal lines extending across the system. An example of the banded structure is shown in Fig. 8 .

The banded structure is used to simplify sweeping through the mesh in the solution algorithm and to simplify specification/generation of the spatial mesh. Figure 8 also illustrates a further restriction on the triangular mesh. The triangles within a band must be arranged such that each interior vertex must be common to exactly three triangles within that band.

o. Card Two, Entry Ten - ITMAX

Estimated number of triangles on any one band.

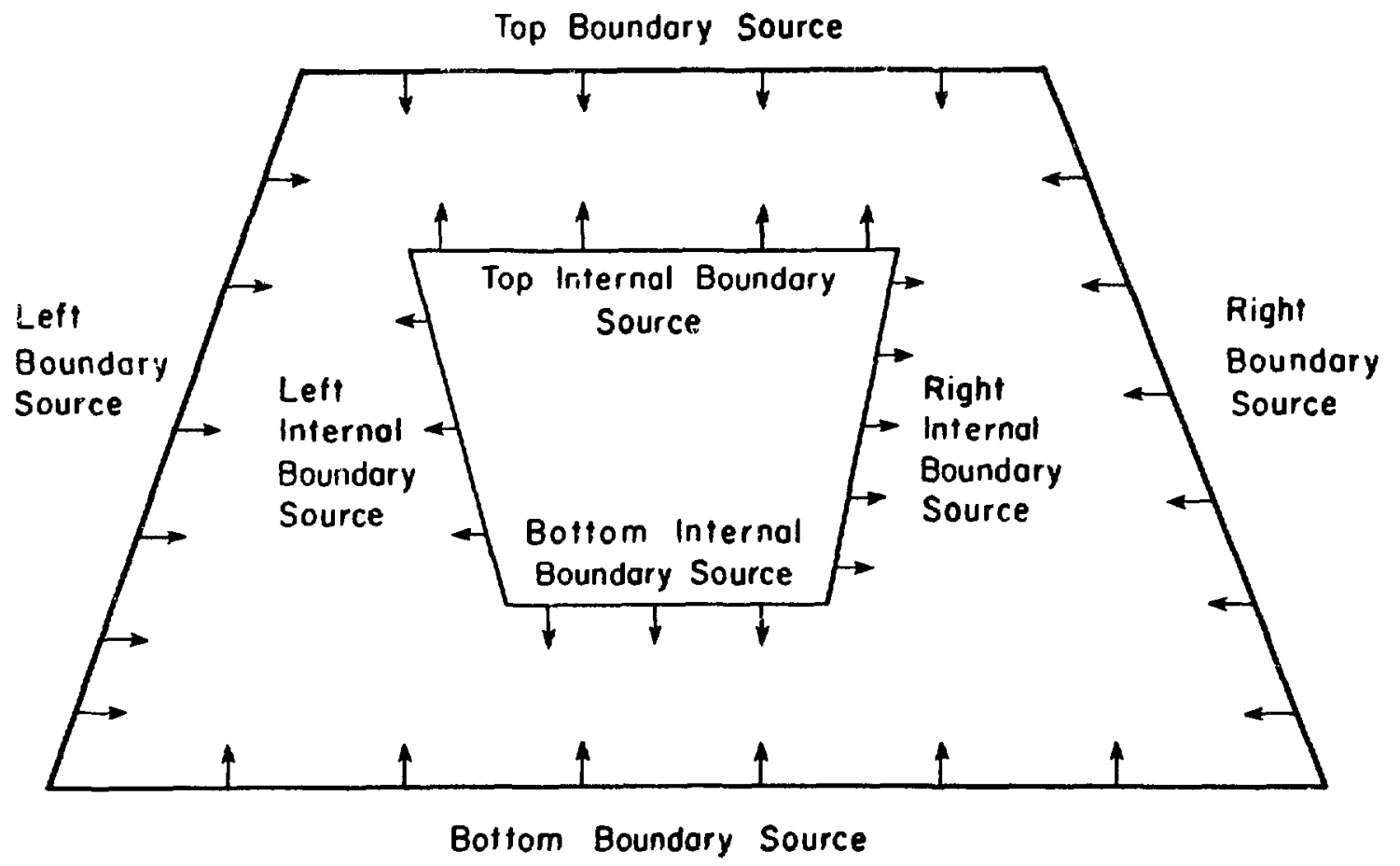

Fig. 7. Boundary source illustration 
To properly allot computer storage for reading in the spatial mesh, an estimate of the maximum number of triangles on any one band must be provided. When the automatic mesh generator is used, up to three triangles per zone can be added to the number requested by the user. The estimate must be a number greater than or equal to the real maximum number or triangles per band. In cases where the spatial mesh is entered by the user, ITMAX is known.

P. Card Two, Entry Eleven - MSHOPT

0/1 (automatic/input triangle vertices) spatial mesh option.

The spatial mesh can be specified by inputing the coordinates of all the triangle vertices or by allowing TRIDENT-CTR to generate vertex coordinates. The mesh generator will take input on a per-band basis: the number of zones on the band, the number of triangles desired per zone, and the coordinates of the zone boundaries. A zone is required to be an area of constant material specification, constant source, and constant "card image" inputed starting flux guess.

The mesh generator basically works by forming a rectangular area in the center of the zone and dividing it with isosceles triangles. To form the rectangular area, the zone boundary slopes are checked. If the slope for a boundary is less than 0.2 , the side is assumed vertical and bounds one side of the rectangle. If the slope is greater than 0.2 , a vertical line is drawn across the band from the innermost point of the zone boundary, forming a triangle with the zone boundary. An example of a spatial mesh created by the mesh generator is given in Fig. 9 . The solid lines are the calculated triangle boundaries. The input number of triangles desired in the region was three. The left-zone boundary slope was greater than 0.2 , so triangle 1 was formed to make the vertical left side of the center rectangular area. The right-zone boundary similarly required the addition of a triangle to form the rectangle area. To maintain the correct orientation of triangle 6, (which because of the zone boundary slope must be up-pointing) an additional triangle was added to the center zone. As a general rule, the mesh generator can add up to three extra triangles per zone over and above the number specified as input.

In some cases the above procedure will leave "holes" in the mesh. When an end triangle, such as triangles 1 and 6 in Fig. 9, have an area larger than four times the typical area of a triangle in the zone, a different procedure is used. In these cases the vertices on the upper and lower sides of the band are spaced equally between the upper and lower band end boundaries.

Inputing a boundary source may require prior knowledge of the vertex coordinates. 


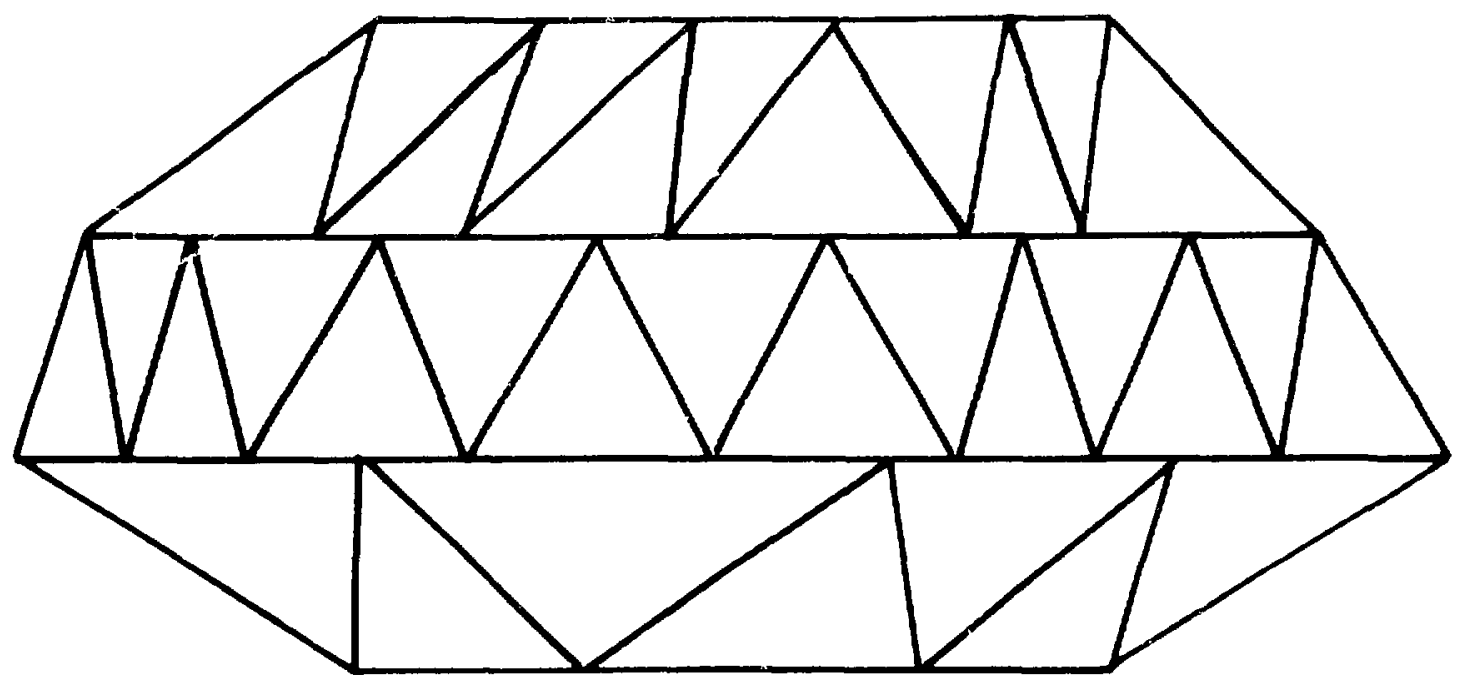

Fig. 8. An example of a TRIDENT-CTR banded triangular mesh.

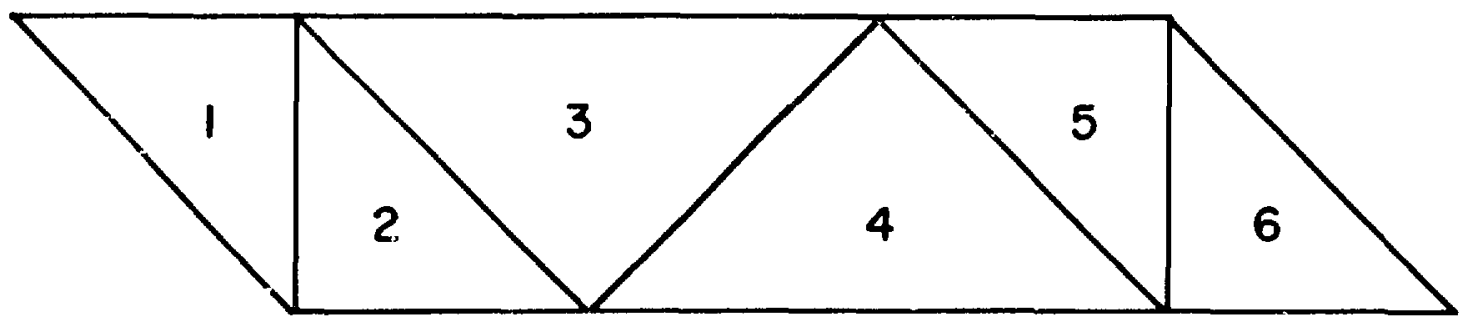

Fig. 9. Automatic mesh generator spatial mesh. 
When such a situation occurs, the user may first run the problem with the ISTOP option (See Sec. III.H) set to -1, (stop after input is complete) and without the boundary source options set. The spatial mesh will be generated and printed out in the input section and the boundary source input can be determined. The problem may then be run using both the automatic mesh generator and the boundary source options.

Q. Card Three, General Comments on Internal Boundary Source Specfication Integers on Entries One through Ten

The location of the internal boundary sources within the problem domain is completely arbitrary, as long as they are located on the sides of triangles in the spatial mesh. As a result, specification of the internal boundaries requires more information than the external boundaries of the problem domain.

To specify the right or left internal boundary source location three pieces of information are required:

(1) the band identification number of the lowest or bottom band that contains the internal boundary source (JLIB or JRIB),

(2) the band identification number of the highest or top band that contains the internal boundary source (JLIT or JRIT), and

(3) the triangle identification number of the triangle that the internal boundary source is entering for each band for which the source is defined (array IDBL or IDBR entered in the problem dependent data section).

In Fig. 10 left and right internal boundary sources are indicated by the heavy black lines and arrows. To specify the left internal boundary source location; JLIB would be set to 2 , JLIT set to 5 , and 2, 3, 3, and 3 entered into the IDBL array in the problem dependent data section. To specify the right internal boundary source location; JRIB would be set to 3 , JRIT set to 4 , and 9 and 9 entered into the IDBR array in the problem dependent data section.

To specify the location of the top or bottom internal boundary source three pieces of information are also required:

(1) the band identification number of the band that the internal boundary source enters (JBBI or JBTI) (in the case of the bottom internal boundary, the source enters from the top of the band and is downward ( $\eta$ ) directed; from the top internal boundary, the source enters from the bottom of the band and is upward $(+\eta)$ directed), 
(2) the number of triangles in the band that contain the source (NBBI or NBTI) (they must all have the same orientation, up or downpointing, and be adjacent), and

(3) the triangle identification number of the first triangle to contain the source (ITBBI or ITBTI).

In Fig. 11 bottom and top internal boundary sources are indicated by the heavy lines and arrows. To specify the bottom internal boundary source location JBBI would be set to 2 , NBBI set to 1 and ITBBI set to 3 . To specify to top internal boundary source locations JBTI would be set to 4 , NBTI set to 2 , and ITBTI set to 2 .

k. Card Four, Entry One MT

Total number of materials (cross section blocks, including anisotropic cross sections) in the problem.

A cross section block is a IHM column by IGH (see Sec. III.V) row array of cross section data. It is assumed in TRIDENT-CTR that blocks of anisotropic cross sections which are used in the calculation have block numbers in ascending sequence, starting with the isotropic cross section block. For example, suppose that block 50 is the isotropic cross section block for hydrogen and 1 SCT $=3$. Then, block 51 must be $\sigma_{s 3}$. If a material is made by mixing two anisotropic scatterers, then the anisotropic blocks must also be mixed with the same densities to form anisotropic blocks for the material.

This number must include both the number of input materials and those materials created by the mix instructions. (See Sec. III.U.)

S. Card Four, Entry Two MTPS

$-\mathrm{N} / \mathrm{N}$ (GPODXS/ISOTXS) number of materials from interface file. CAUTION: Material set from this file yields ISCT+1 materials.

TRIDENT-CTR accepts cross section data from the standard file ISOTXS, ${ }^{11}$ and a file GPODXS, created by the Los Alamos code MIXIT. The ISOTXS file is a standard interface file developed for use by the fission reactor community.

The GPODXS file is group ordered cross section file. This file can be used if the cross section data is too large to fit into SCM. TRIDENT-CTR normally reads in all the cross section data into SCM, manipulates it, and writes it out to LCM in group-ordered blocks. The GPODXS file already has the cross section data organized in group-ordered blocks that may be read into SCM a group at a time. 


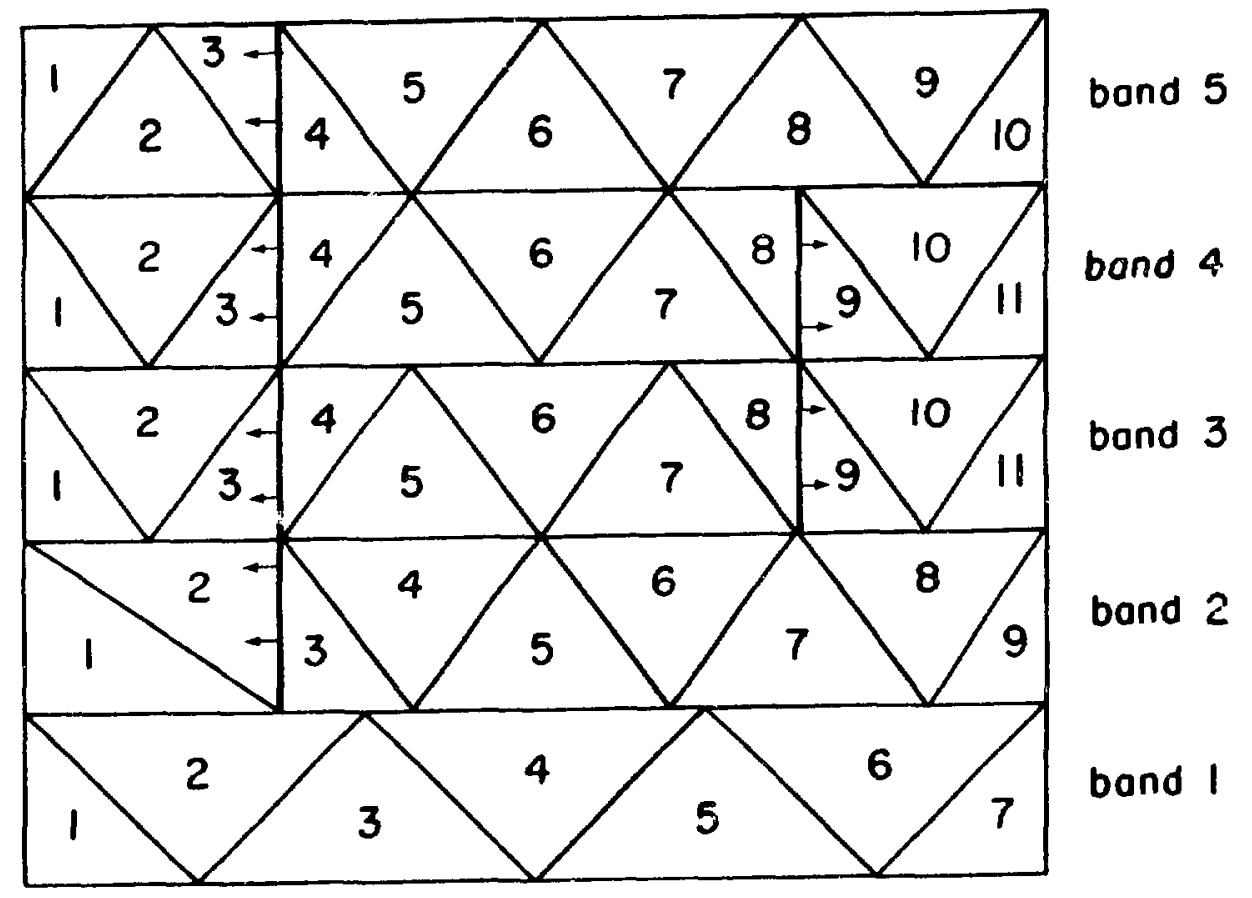

Fig. 10. Specification of the left and right internal boundary source location.

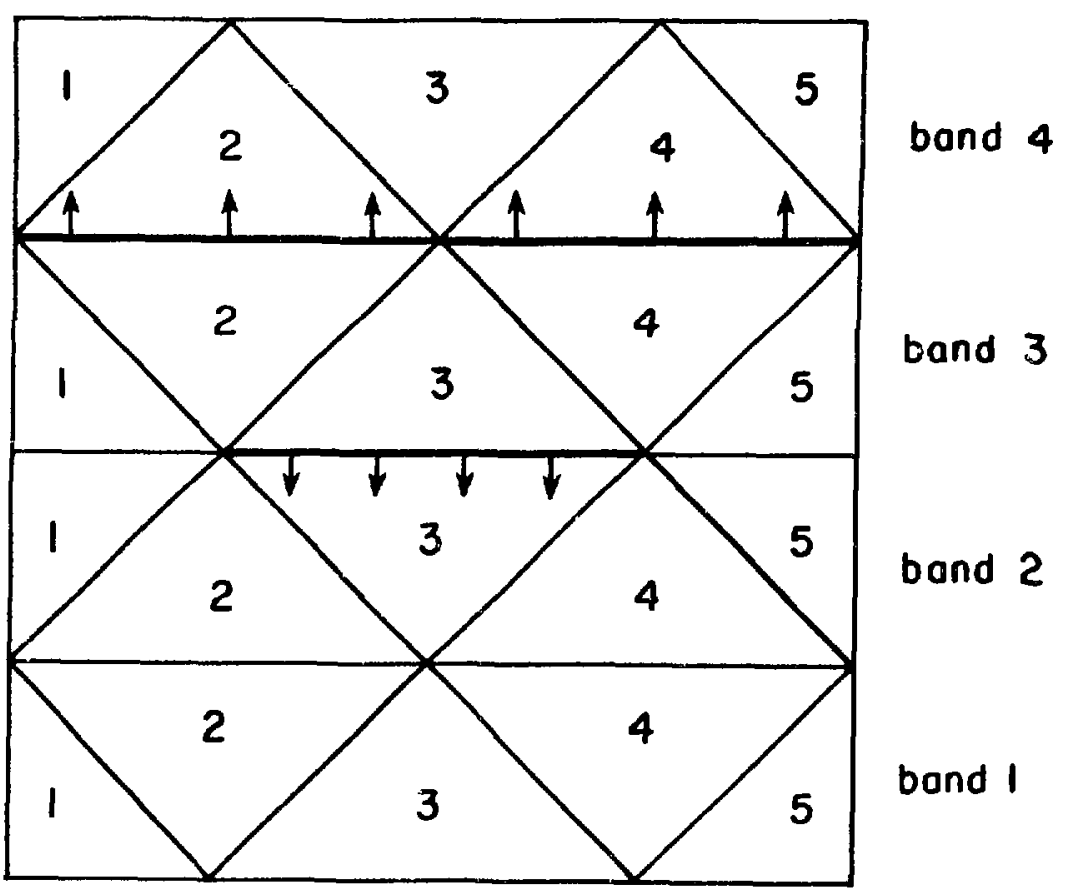

Fig. 11. Specification of the bottom and top boundary source locations. 
T. Card Four, Entry Three MCR

Number of materials from code-dependent (card image) input file. If this number is negative, FIDO ${ }^{12}$ format cross sections are used.

MCR sets the number of materials that will be read in from cards or card images. The FIDO format for cross sections is equivalent to the ANISN cross section format.

\section{U. Card Four, Entry Four MS}

Number of mixture instructions.

The user is free, in TRIDENT-CTR, to enter macroscopic cross sections and bypass the mixing algorithms; specification of the input value MS $=0$ is all that is required for this. If $M S \neq 0$, the user must provide three sets of MS numbers which are stored in the vectors MIXNUM, MIXCOM, and MIXDEN. These numbers are used in the following algorithm to manipulate cross section blocks:

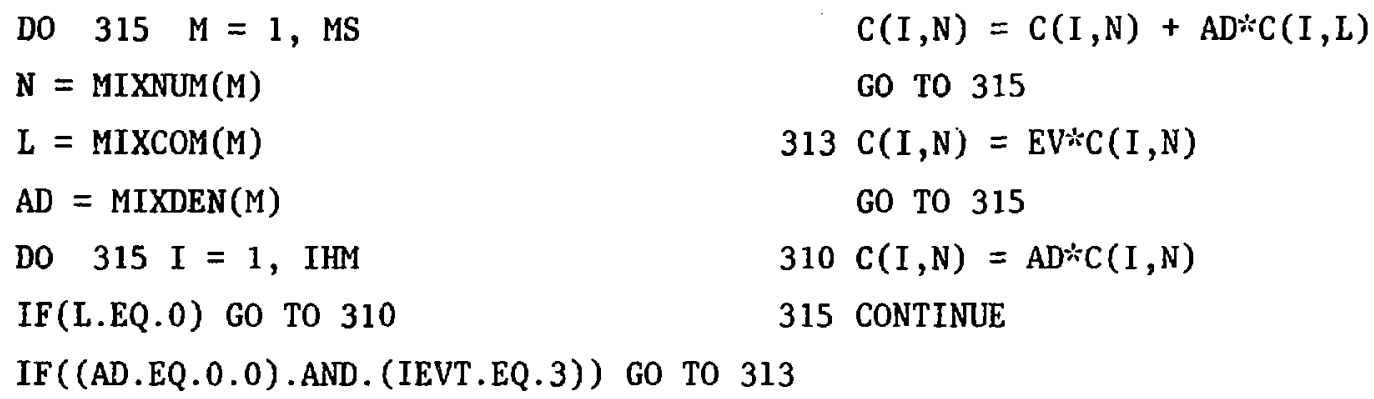

In this algorithm, cross section block $N$ is created or altered by adding multiples of block $\mathrm{L}$ or by multiplying the block $\mathrm{N}$ by a factor. Let us consider some examples.

Suppose we have entered 45 cross sections as input. Then any mixtures that are made must be given block numbers higher* than 45. For example suppose we enter the constants of Table IV. For this example we have MS $=17$ instructions. In the first three instructions, block 46 is cleared (set to zero) and then made up of 0.0478 parts of block 1 and 0.0333 parts of block 20 . If blocks 1 and 20 are microscopic cross sections in barns, then 0.0333 and 0.0478 times $10^{24}$ are the atomic densities. In the second set of instructions, block 47 is cleared and then made up of 0.1179 times the result of adding three-fourths of block 2 to one-fourth of block 3 . In the next set of instructions block 48 is cleared and

\footnotetext{
*To preserve the input values. If these need not be saved, mixtures can be created in lower block numbers.
} 
TABLE IV

SAMPLE MIXTURE-SPECIFICATION PARAMETER

\begin{tabular}{lll}
$\begin{array}{c}\text { MIXNUM } \\
(N)\end{array}$ & $\begin{array}{c}\text { MIXCOM } \\
(\mathrm{L})\end{array}$ & \multicolumn{1}{c}{$\begin{array}{l}\text { MIXDEN } \\
(\mathrm{AD})\end{array}$} \\
\cline { 2 - 3 } 46 & 0 & 0.0 \\
46 & 1 & 0.0478 \\
46 & 20 & 0.0333 \\
& & \\
47 & 0 & 0.0 \\
47 & 2 & 0.75 \\
47 & 3 & 0.25 \\
47 & 0 & 0.1179 \\
48 & & \\
48 & 0 & 0.0 \\
48 & 15 & 0.0049 \\
48 & 14 & 0.0078 \\
49 & 48 & 0.0 \\
49 & & \\
49 & 0 & 0.0 \\
49 & 33 & 0.5 \\
49 & 34 & 0.5 \\
49 & 0 & 0.187 \\
& 49 & 0.0 \\
46 & 4 & 0.1
\end{tabular}

made up of portions of blocks 15 and 14 . If IEVT (the input eigenvalue type option) is 3 , then the resulting block 48 is multiplied by EV (the input eigenvalue guess). In this type of problem the program attempts to find a value of EV such that the resulting concentration of block 48 renders the system critical. If IEVT $\neq 3$, the line of instructions $48,48,0.0$ would not alter the composition of block 48. In the final sequence, block 49 is made up of 0.187 times one-half of block 33 and block 34, and provision is made to search for the concentration of this portion of 49 to which is always added 0.1 of the previously mixed block 46. It should be clear that there are many possibilities not covered in these examples, but by examining the FORTRAN Instructions above, the user should be able to prepare his own sets of mixture instructions. 
IHT is the row of the total cross section, IHS is row of the within group scattering cross section, and IHM is the total number of rows in the cross section format.

The Los Alamos cross section format assumes that each nuclide is described by a block of cross sections of IHM rows for IGM group columns. The row position of cross sections is specified relative to the total cross section, $\sigma_{t}$ (row IHT), and the within-group scattering cross section, $\sigma_{s, g \rightarrow g}$ (row IHS). It is assumed that the row order of the cross sections is as indicated in Table $V$.

In this format, group $\mathrm{g}+1$ corresponds to a group of lower energy than group g. The symbol $\sigma_{s, g-2 \rightarrow g}$ denotes the scattering transfer probability from group $g-2$ to group $\mathrm{g}$. The format allows $N$ groups of upscatter and $M$ groups of downscatter (i.e., the scattering matrix need not be symmetric). However, all cross-section blocks must have the same values for IHM, IHS, and IHT. The fission cross section, $\sigma_{f}$, times the mean number of neutrons per fission, $v$, must be located in row IHT-1, and the absorption cross section, $\sigma_{a}$, must be entered in row IHT- 2 . If a scattering matrix is used to represent $(n, 2 n)$ reactions, that is, if $\sigma_{s, g \rightarrow h}$ contains 2 times the $(n, 2 n)$ transfer probability $\sigma_{n, 2 n ; g \rightarrow h}$, then the value

$$
\sigma_{n, 2 n}=\sum_{a 11 h} \sigma_{n, 2 n ; g \rightarrow h} \text {, }
$$

must be entered in IHT-4. The user is free to enter additional cross sections at the top of the format. These extra cross sections are not used in the calculation, but are available for reaction-rate computations after the particle flux is obtained. It is sometimes convenient to locate the transport cross section, $\sigma_{t r}$, in IHT-3 and use this cross section instead of $\sigma_{t}$ in the calculation of buckling corrections.

In upscattering problems, the program does not need the special $\sigma^{\text {up }}$ cross section which is required in earlier Los Alamos programs. 8 In TRIDENT-CTR, it is assumed that $\sigma^{\text {up }}$ is present, and $\sigma^{\text {up }}$ is automatically removed from card input cross section sets unless the user tags the input number IHT with a minus sign. Cross sections read with the FIDO format may not contain $\sigma^{\text {up }}$.

W. Card Four, Entry Eight IQAN

Order of anisntropy of the inhomogeneous source.

For a detailed description see Sec. III.I. When using the anisotropic-distributed-source option, the order of anisotropic scattering, ISCT, must be at 


\section{TABLE V}

CROSS SECTION INPUT WITH

LOS ALAMGS FORMAT

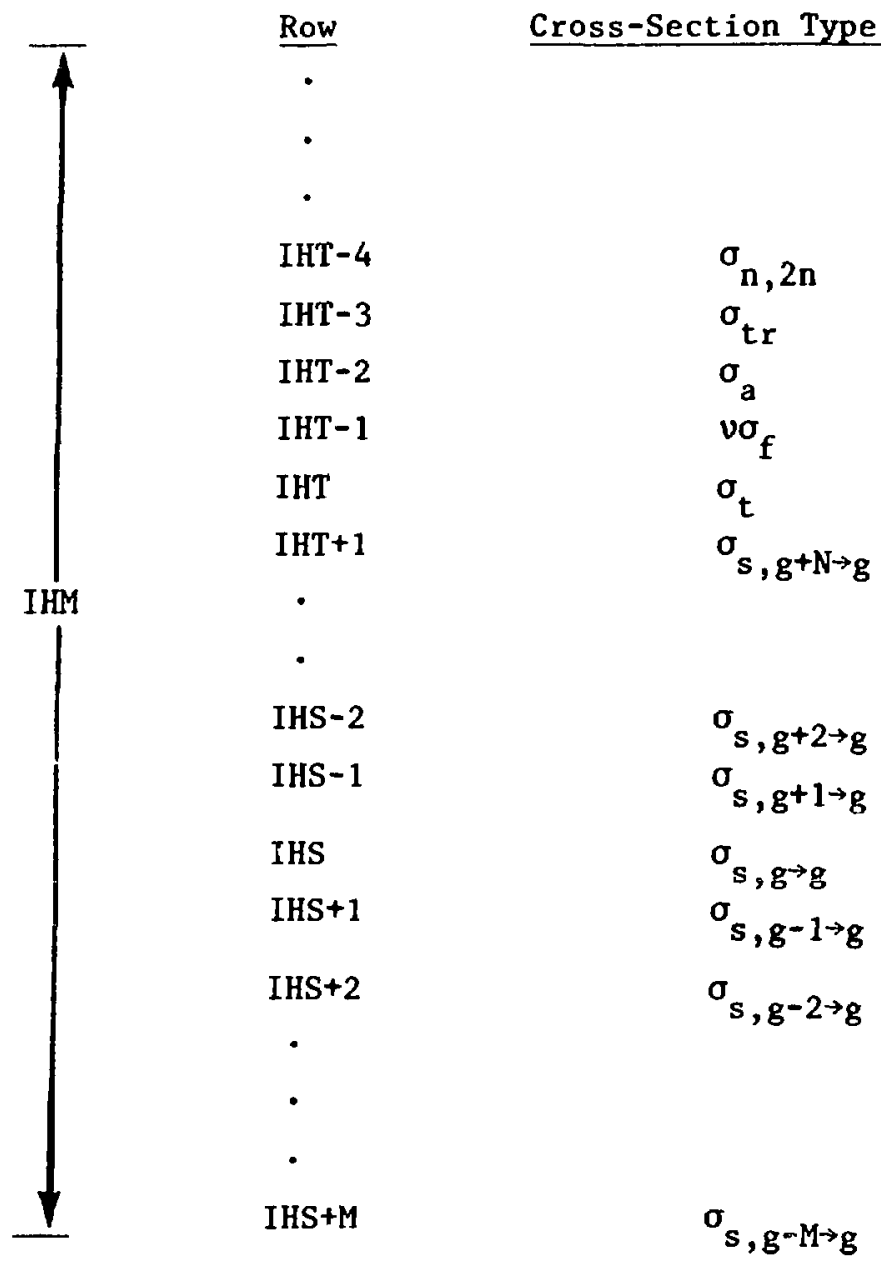

least as large as IQAN so that the correct number of spherical harmonic polynomials are computed.

X. Card Four, Entry Nine IPVT

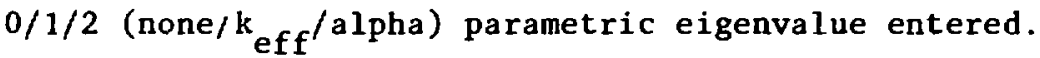

It is possible in TRIDENT-CTR to adjust nuclide concentrations or the value of the time absorption to achieve a desired value of $k_{\text {eff }}$. This value is taken to be unity (criticality) unless the parametric eigenvalue trigger (IPVT) is set 
to unity. In this case, the parametric value of $k_{\text {eff }}$ is entered as an input number. If IPVT $=2$, a $1 / v$ absorber of value $P V$ is added to the problem in each space cell. See Sec. III.F for detailed description of eigenvalue searches.

Y. Card Four, Entry Ten ITTL

Maximum number of inner iterations allowed per group.

TRIDENT-CTR uses a dual iteration strategy for solving the discretized transport equation. The two nested iterations are referred to as outer and inner iterations. The outer iteration represents a sweep through all the energy groups while the inner iteration is performed within each group across the space-angle mesh. Z. Card Five, Entries One through Five

These five entries are used to specify the angular quadrature coefficients. TRIDENT-CTR allows specification of different quadrature $\left(S_{N}\right)$ coefficients for different energy groups (group-dependent quadrature). The quadrature $\left(S_{N}\right)$ coefficients may be either the built-in sets, sets read in off card images, or a combination of built-in and user-inputed sets.

The built-in sets are Carlson's $\mathrm{EQ}_{\mathrm{N}}{ }^{13}$ sets and are symmetric under $90^{\circ}$ rotations for reflective boundary conditions and $r-z$ geometry symmetry requirements (see Sec. III.B and $M$ ). When the fictitious source option is used, the built-in sets must be used.

The first three entries on card five are used to properly dimension the quadrature arrays.

(1) MISN is the maximum $S_{N}$ order used in any energy group. MISN should be an even number such that $2 \leqq M I S N \leqq 16$. If only one $S_{N}$ set is used for all groups and the set is to be read in, then MISN must be negative and equal to the total number of directions in the user entered $S_{N}$ set.

(2) MMNP is the maximum number of $S_{N}$ directions in any one quadrant in any one $\mathrm{S}_{\mathrm{N}}$ set. In Fig. 12 the number of $\mathrm{S}_{\mathrm{N}}$ points per quadrant (NPQ) is the same for all four quadrants and equal to 6 , hence MMNPQ is equal to 6 . In Fig. 13 the number of $S_{N}$ points per quadrant is different for the upper and lower quadrants, with MMNPQ equal to six.

(3) MREL is the maximum number of eta levels in any one quadrant in any one $S_{N}$ set. In the case of a read-in $S_{N}$ set, the code sorts the $S_{N}$ set into eta levels. If any two $S_{N}$ points in a read-in set differ by more than 
$1.0 \times 10^{-6}$, they are put in different levels. In Fig.

13 the number of eta levels in quadrants 1 and 4 is three, and quadrants 2 and 3 it is 2 . Hence MMEL would be 3 .

ISNOPT, the 4th entry, indicates whether group dependent quadrature, one built-in $S_{N}$ set, or one card inputed $S_{N}$ set will be used. If group dependent quadrature is used, then it can be a mix of built-in or card inputed sets and appropriate specification is made in the problem dependent data section (see Sec. III.CC).

NSN, the 5 th entry, specifies the number of $\mathrm{S}_{\mathrm{N}}$ sets to be used in the problem. For ISNOPT $=-1$, it must be greater than one; for ISNOPT=1, NSN=1

AA. Card Five, Entries Six aid Seven

TRIDENT-CTR can produce several binary files that can be post processed to provide graphic displays. The post processors are relatively simple programs that can be easily modified to meet specific user requirements.

If IMSHPLT is set to one, a binary file called MSPLT is produced that contains the information required to display the spatial mesh with material identification number inserted in the triangles (the triangles in which the numbers will fit). By

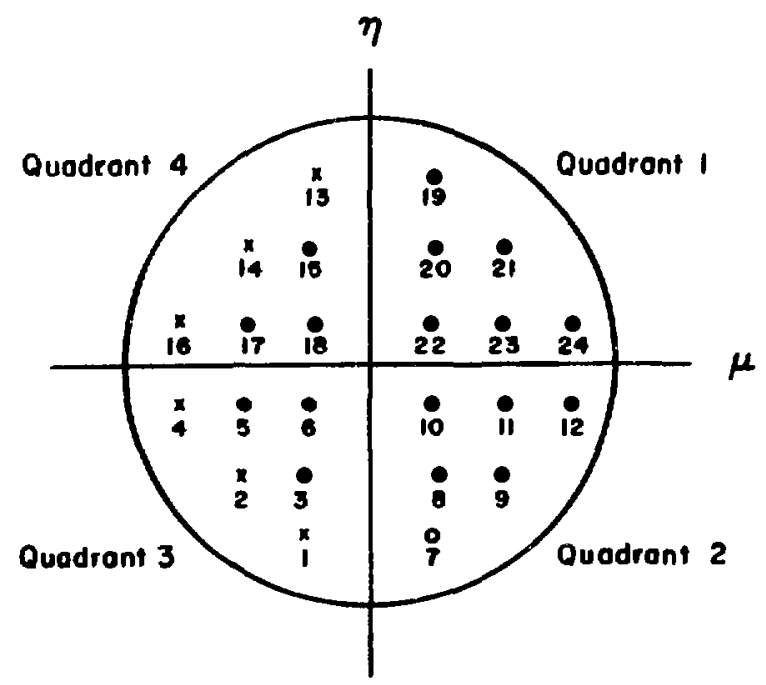

Fig. 12. Built-in $\left(\mathrm{EQ}_{6}\right)$ quadrature directions.

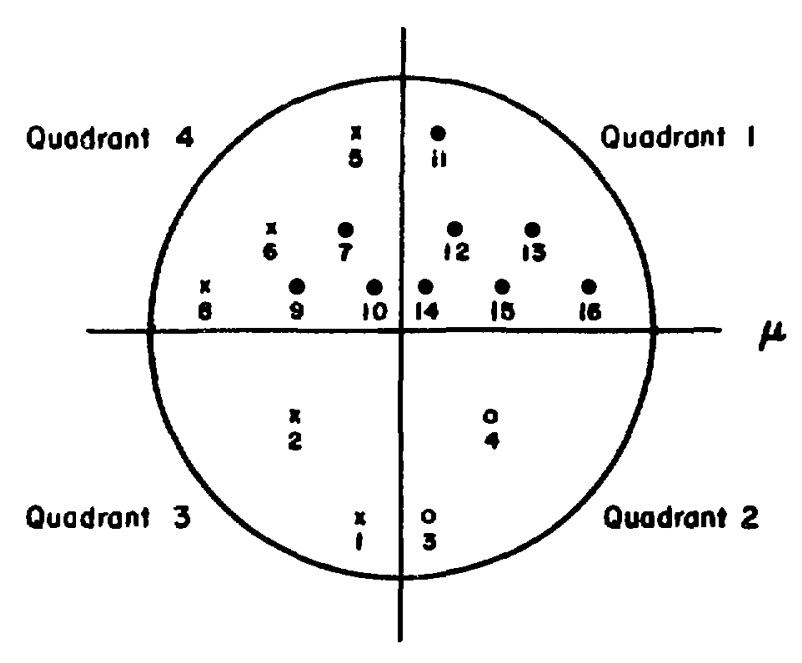

Fig, 13. Sample input quadrature directions. 
using the post processor TRD2D, all or any rectangular subregion of the spatial mesh will be displayed.

By setting IEDPLT to 1 , binary files are created that will display threedimensional plots of the material mesh, fluxes, and response functions. Depending upon data entered in the problem dependent section of code, files called MATPLT, FLXPLT, and RESPLT are created that can be post processed by TRD3D. Again either the entire problem domain or specified rectangular subregions may be displayed.

In the material 3-D plots (MATPPLT), the problem domain or a specified subregion is represented by one level and a specific material or set of materials by another level. The 3-D flux plots (FLXPLT) show the variation of group or groupsummed fluxes across the problem domain or a specific subregion. The 3-D response function plots (RESPLT) show the spatial variation of a specific cross section or set of cross sections multiplied by the flux and summed over specified number of energy groups.

BB. Card Six, Entry One ITLIM

$0 / \mathrm{N}(\mathrm{no} / \mathrm{N}$-second) time limit dump.

If an integer number of seconds is entered a flux dump taken after this number of seconds and the problem is terminated. For large long running problems, the user may want to run the problem in segments. This may be done by using the ISTOP control integer (See Sec. III.H) and setting specific number of groups to be solved, or using ITLIM to use a time limitation. Then by using the flux dumps, the ISTART control integer (see Sec. III.G), and the JSTART control integer (see Sec. III.L) the problem may be restarted at a later time.

CC. Card Six, Entry Two IEDOPT

$0 / 1$ (no/yes) special edit input to be entered.

The TRIDENT-CTR user is provided with two types of edit options that provide printed output zone edits and point edits. As many different zone and point edits as desired may be performed.

1. Zone Edit

An edit zone is a collection of triangles which have the same zone number. The user defines a zone by entering a set of NTC numbers (NEDZ array) which associates with each triangle of the fine mesh a zone identification number (zone j.d.). The triangles of an edit zone need not be contiguous. For each group and zone, a table containing the zone volume, net leakage, buckling absorption, 
source, integral flux, average flux, and macroscopic activities (for cross section positions one through IHT) is given. The macroscopic activity $A_{k}(g, I P O S)$ in zone $k$ and group $g$ for cross section position IPOS is defined by

$$
A_{k}(g, I P O S)=\sum_{i} c\left(g, I P O S, m_{i}\right) \phi_{i} V_{i} \text { for } i \varepsilon \text { zone } k
$$

where $m_{i}$ is the material i.d. (cross section block identification number) for triangle $i, C(g, I P O S, m)$ is the cross section for group $g$ in position IPOS for material $\mathrm{m}, v_{i}$ is the triangle volume, and $\phi_{i}$ is the average flux in triangle $i$. Thus $A_{k}$ is the activity computed with the macroscopic cross section actually used in the problem, sumed over all triangles in zone $k$.

For each zone edit, the TRIDENT-CTR user is provided the option of calculating constituent activities and microscopic activities for any material desired. The constituent activity $A_{k}^{j}(g$, IPOS) for material $j$ in zone $k$ is defined by

$$
A_{k}^{j}(g, I P O S)=\sum_{i} C\left(g, \text { IPOS }, m_{i}\right) \phi_{i}{ }^{\prime} \delta_{j m i} \text { for }{ }^{i} \varepsilon \text { zone } k
$$

Here $\delta_{j m i}$ equals unity if material $j$ equals material $m_{i}$, the mixture table density (MIXDEN) if material $j$ is a "constituent" of material $m_{i}$, and is zero otherwise. A "constituent" means that material $j$ appears as an entry in the MIXNUM array with density MIXDEN (see Sec. III.U) that is used to form material $m_{i}$. Thus if material $j$ is used to form a material $j$ ', which is used to form material $m_{i}$, then material $j$ is not a "constituent" of of material $m_{i}$ within this definition. The microscopic activity for mateiral $j$ in zone $k$ is defined by

$$
A_{k}^{j}(g, I P O S)=\sum_{i} C(g, I P O S, j) \phi_{i} V_{i} \text { for } i \varepsilon \text { zone } k
$$

Thus $A_{k}^{j}$ would be the activity obtained in zone $k$ if material $j$ were uniformly distributed throughout the system, even though material $j$ may not actually have appeared in the problem cross sections. 
The edit input parameters NCA and NMA specify the number of constituent activities and number of microscopic activities to be calculated. The user must then enter NCA material i.d.'s for the constituent activities and NMA material i.d.'s for the microscopic activities.

To edit a material which is not actually a part of the problem, the TRIDENTCTR user may add a mixture instruction to the mixture tables; or, if interested in only a few cross sections, he may add these cross sections to other blocks in rows IHT-5, IHT-6, etc.

Finally, following any constituent activities or microscopic activities, the zone edit provides the zone relative fission source rate density (group sum of the zone volume integral of $v \sigma_{f}$ fission rate divided by the zone volume), normalized to that of a user-designated zone. The zone relative fission source rate density (unnormalized) is defined by

$$
\mathrm{PD}_{\mathrm{k}}=\frac{\sum_{\mathrm{g}} \sum_{i} \mathrm{c}\left(\mathrm{g}, \mathrm{IHT}-1, \mathrm{~m}_{\mathrm{i}}\right) \phi_{i} \mathrm{v}_{\mathrm{i}}}{\sum_{i} \mathrm{v}_{\mathrm{i}}} \text { for } \mathrm{i} \varepsilon \text { zone } k
$$

If the user selects zone zero (NORMZ=0), the normalization is to the whole system fission source rate density.

\section{Point Edit}

The point-edit feature of TRIDENT-CTR provides the user with the option of obtaining the pointwise variation across each triangle of the scalar flux and activities. The user must identify the triangles over which the point edits are desired. For each point edit, a map of the point arrangement indexing on a triangle is printed by subroutine ARRMAP. This is followed by the pointwise scalar flux for the specified triangles. The user is also provided by the NPMA (number of point microscopic activities) parameter the option of obtaining a pointwise microscopic activity for material $j$ in triangle $i$ at point $k$ as

$$
A_{k i}^{j}(g, I P O S)=c\left(g, I P O S, m_{j}\right) \phi_{k i}
$$

where $\phi_{k i}$ is the scalar flux at point $k$ in triangle $i$. 
DD. Card Six, Entry Three Through Seven

The control integers for these entries are used to limit the amount of data printed. Often the entire output of all the final moments is not desired. For many cases only the scalar flux is useful. Likewise, once a printout of the input source, flux, or cross sections has been made, it is not needed in subsequent runs oi the same problem.

(1) With entry three, Il, set to zero, the input flux will not be printed.

(2) With entry four, IFTP, set to zero, all final-flux moments are printed. When set to one, only the isotropic flux is printed and when set to two, none of final flux is printed.

(3) With entry five, I3, set to zero, all the cross section sets are printed. When 13 is set to one, only those cross section sets created by the mix instructions is printed, and when set to two, no cross sections are printed.

(4) With entry six, I4, set to zero the final fission source $\left(v \sigma_{f} \phi\right)$ is printed. With $\mathrm{I} 4$ set to one the fission source is not printed.

(5) Entry seven, I5, controls the source print. The entries $0 / 1 / 2 / 3$ respectively yields a print out of both the unnormalized source and the normalized source; print of only the unnormalized source; of prints only the normalized source; and a print of no sources.

[Note: Entry eight on card six is not used.]

EE. Card Six, Entry Nine, ISDB

0/1 (whole system/space dependent) buckling height indicator.

Buckling heights (in $\mathrm{cm}$ if cross sections are in barns) are used to simulate the $z$-dimension of an $x-y$ geometry problem or $\theta$ in $r-z$ geometry by adding an absorption given by

$$
\sigma_{a, B H T}=\frac{\sigma_{t}}{3}\left[\pi /\left(B H T * \sigma_{t}+1.4209\right)\right]^{2}
$$

Here 1.4209 is twice the Milne problem extrapolation distance, and $\sigma_{t}$ is the total cross section. For $\mathrm{ISDB}=0$, a single, whole-system buckling height is entered. For ISDB $=1$, a buckling height is entered for each triangle. For BHT = 
0 , no buckling absorption is added.

[Note: Entry ten on card six is not used.]

FF. Card Six, Entry Eleven IDUMP

$0 / 1 / 2$ (name/scalar/total) final flux dump.

When a problem has been run to completion, the user may want only the final scalar flux. For large problems with a high order anisotropic scattering approximation, the size of a scalar flux file can be much smaller than a total flux file (e.g.for $\mathrm{P}_{3}, 1 / 10$ the size). It should be pointed out that if a user wishes to perform edits or plot edits at a later time with the finai scalar flux, he may re-run this problem, with the starting flux guess taken from the isotropic flux file, (ISTART $=-5,-6$, or -7 ; see Sec. III.G) skip the computation overlays (set ISTOP $=-1$, see Sec. III.H) and go to the edit portion of the code. So by saving a scalar final flux file (IDUMP $=0$ ) the user retains future edit capability with a saving of storage space, and lower computer time requirements.

GG. Card Six, Entry Twelve ITTY

0/1 (no/yes) TTY output options available.

Certain portions of TRIDENT-CTR's output are available at a terminal (e.g., final fluxes) on an interactive basis. When ITTY is set to one, the code will prompt the user concerning his necd for certain infomation at the terminal. In these cases the user must enter yes or no from the terminal. When running in a batch mode, ITTY must be set to zero, to insure the problem runs to completion. HH. Card Seven, Entries One Through Six

Card seven is used to enter data for eigenvalue searches. The methodology used in searches is described in detail Sec. III.F. For IEVT $\leq 1$, all zeros may be entered on this card.

$\mathrm{EV}$ is the starting guess for the eigenvalue, and EVM is the eigenvalue modifier as described in Sec. III.F.

To display the relationship between the input parameters IEVT, IPVT, and PV, and the output eigenvalue (EV), Table VI is provided. The parameters XLAL, XLAH, and XLAX are also described in detail in Sec. III.F.

II. Card Eight, Entry One EPSO

There are three levels of iterative processes in the TRIDENT-CTR program: (1) the inner iteration, in which the within-group scattering source and/or the fictitious source, and/or the boundary flux at an implicit boundary, change; 
TABLE VI

COMBINATIONS OF IEVT, IPVT, PV, AND EV

\begin{tabular}{|c|c|c|}
\hline IEVT & $\underline{\text { IPVT }}$ & MEANING OF EVout \\
\hline 2 & 0 & System $\alpha$, no PV required \\
\hline 2 & 1 & $\alpha$ poison added to system to yield a system $k_{\text {eff }}=P V$ \\
\hline 2 & 2 & $\alpha$ poison added to system such that $E V+P V=\alpha_{\text {sys }}$ \\
\hline 3 & 0 & $\begin{array}{l}\text { density multiplier required to make system } k_{\text {eff }}=1.0 \text {, } \\
\text { no } P V \text { required }\end{array}$ \\
\hline 3 & 1 & density multiplier required to make system $k_{\text {eff }}=P V$ \\
\hline 3 & 2 & density multiplier required to make system $\alpha=P V$ \\
\hline
\end{tabular}

the outer iteration in which the fission or upscattering source changes or which is caused by an artificial inner iteration limitation (usually in inhomogeneous source problems); and (3) the parametric eigenvalue search iteration in which, after a converged outer iteration, the value of a material concentration or a time absorption is changed.

Two convergence precisions are input: EPSO and XLAX. In a parametric eigenvalue search, two values of ( $r$ indicates iteration number)

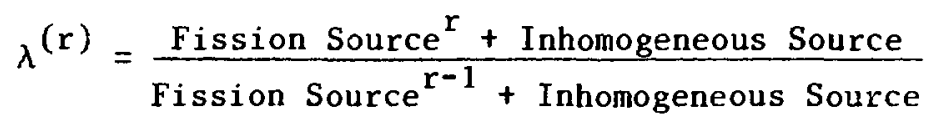

are required to differ by less than XLAX before a new eigenvalue guess is computed. All other processes are tested against precisions derived from EPSO. These precisions are:
EPSO
outer iteration convergence
EPSI $=$ EPSO
inner iteration convergence
$\operatorname{EPSR}=10^{-4}$
rebalance number
$\mathrm{EPSX}=\mathrm{EPSO}$
collapsed group convergence 
In the inner iteration processes, we require

$$
\max \left|1-\frac{\phi_{i}^{r-1}}{\phi_{i}^{r}}\right|<\operatorname{EPSI}
$$

for the $r^{\prime}$ th iteration where $\phi_{i}$, is the average scalar flux in the $i$ 'th triangle before application of the rebalance factor. If the number of iterations is greater than the input value IITL, the inner iteration is terminated.

For the iterative computation of the rebalance factor in the inner iteration, we require

$$
\left|1-\frac{\hat{\mathbf{r}}^{\mathrm{r}-1}}{\mathbf{f}^{\mathbf{r}}}\right|<\operatorname{EPSR} \text {. }
$$

In the outer process, if both

$$
|\lambda-1|<\text { EPSO and }|1-\mathbf{f}|<10 \% \text { EPSX, }
$$

the problem is terminated after one final outer iteration. In the rebalance iterative process (denoted with iterative superscript $r$ ) we require

$$
\left|\lambda_{\text {rebalance }}^{(r)}-1\right|<\operatorname{EPSX},
$$

if IEVT $=1$ ( $k_{\text {eff }}$ calculations) and

$$
1-\lambda_{\mathrm{RB}}^{(\mathrm{r})} / \lambda_{\mathrm{RB}}^{(\mathrm{r}-1)}<\operatorname{EPSX},
$$

if IEVT $\neq$ (parametric eigenvalue or inhomogeneous source plus fission problems).

JJ. Card Eight, Entry Two NORM

Normalization factor, the total number of source or fission particles are normalized to this number. No normalization is made if NORM is zero. 
If IEVT is less thas ', the total number of source particles, includiug all boundary sources, is calculated. Then NORM/(total source) multiplies all sources $_{5}$ (volume distributed and boundary). For IEVT $\geq 1$, the fission source is summed and NORM/(total fission source) multiplies the fission source array.

KK. Card Eight, Entry Three POD

Parameter oscillation damper used in eigenvalue searches. The use of POD is described in detail in Sec. III.F.

LL. Entering the Spatial Mesh

The triangular mesh used by TRIDENT-CTR is not completely arbitrary, but is a structured mesh with the following characteristics:

(a) it is banded such that all triangle vertices are constrained to lay on horizontal lines extending across the system, and

(b) each interior vertex within a band of triangles must be common to exactly three triangles withis that band.

The mest used by TRIDENT-CTR differs from that used by TRIDENT ${ }^{1}$ and TRIPLET ${ }^{4}$ in that the vertices on one band have no required relationship with the vertices on adjacent bands. Figures 14 and 15 show a sample problem and a triangular mesh arrangement for the problem. (The numbers within the triangle are material identification numbers.)
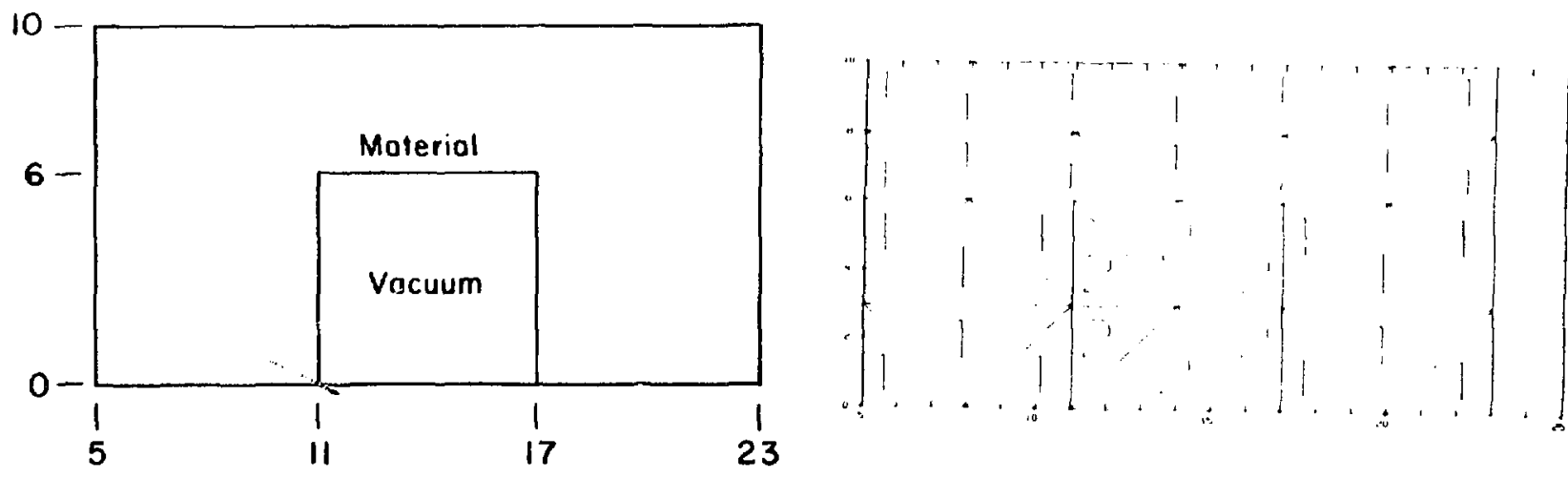

Fig. 14. Sample problem for TRIDENTCTR.

Fig. 15. Triangular mesh for sample problem. 
Note that characteristic (b) above has the following physical interpretations:

(1) To trace the triangles within a band, place a pencil at a starting point on the left and trace all the nonhorizontal sides continuously without lifting the pencil or retracing.

(2) Using the definition of up-pointing and down-pointing of Fig. 16, an interior up-pointing triangle on a band must be adjacent to two down-pointing triangles and vice versa.

The significance of the above discussion is that to define a band of triangles the following information is required:

(1) the band height or width ( $\Delta y$ or $\Delta z$ )

(2) the orientation of the first triangle on the band (uppointing or down-pointing), and

(3) the $x$ or $r$ coordinates of the vertices ordered in sequence of the connecting sides.

The bands are ordered with increasing y or $z$ coordinate (numbered from bottom to top) and the mesh is constructed by building bands of triangles on top of previously constructed bands.

The user of TRIDENT-CTR has the option of using an automatic mesh generator or specifying all triangle vertices. A description of the mesh generator is contained in Sec. III.P. Basically, the mesh generator requires the band heights, the number of zones per band, the orientation of the first triangle on each band, the number of triangles per zone, and $x$ (or $r$ ) coordinates of the zone boundaries. The vertex input option requires the band heights, the number of triangles on each band, the orientation of the first triangle on each band, and the $x$ (or $r$ ) coordinates of the triangle vertices.

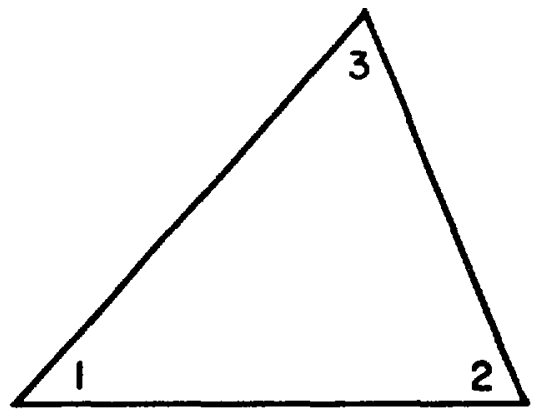

Up - pointing

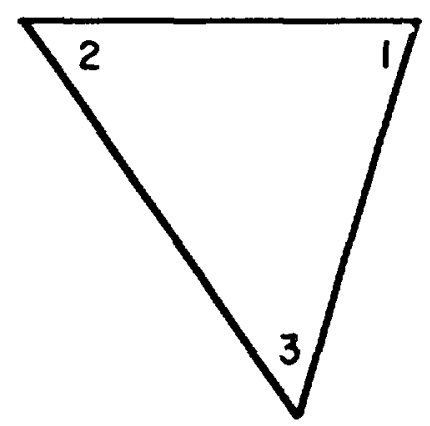

Down-pointing

Fig. 16. Up-pointing and down-pointing triangles. 
For either MSHOPT $=0$ or 1 (automatic/enter vertices) the first entry of the spatial mesh is the band heights ( $H Y(J))$ for all the bands starting with the bottom-most band. JT floating point values are entered with the LOAD format.

For both MSHOPT options the data is loaded in "band blocks." The data required for each band is grouped together, such that there are JT blocks or groups of card or card images entered.

For MSHOPT $=0$, (automatic mesh generator) there are three basic data entries for each band block, $\mathrm{J}$.

(1) A card with two integer entries on a 2 I6 format (ITZ(J) and ITT(J)) is used. The first integer (ITZ $(J)$ ) represents the number of zones on band $J$ and the second integer (ITT(J)) sets the orientation of the first triangle on the band. If $\operatorname{ITT}(J)=1$, the first triangle is up-pointing and if $\operatorname{ITT}(J)=2$, the first triangle is downpointing. (The LOAD format is not used.)

(2) A card or set of cards is read with integer entries on a 1216 format representing the number of triangles per zone. There are ITZ(J) entries ordered with zones going from left to right. (The LOAD format is not used.)

(3) The $2 *(\operatorname{ITZ}(\mathrm{J})+1)$ zone boundaries $\mathrm{x}$ ( or $\mathrm{r}$ ) coordinates are entered with the LOAD format. The zone coordinates are ordered from bottom to top, left to right, with the bottom leftmost coordinate entered first, the top leftmost coordinate entered second, and ending with the top rightmost zone boundary. Zone boundaries may not overlap, but they may touch on the top or bottom of the band to form a one triangle zone.

For MSHOPT = 1, (enter all vertices) there are two basic data entries for each J'th band block.

(1) A card with two integer entries on a 2 I6 format (IT(J) and ITT(J)) is used. The first integer (IT(J)) represents the number of triangles on band $J$ and the second integer (ITT $(J)$ ) sets the orientation of the first triangle on the $J$ 'th band. If $\operatorname{IrT}(J)=1$, the first triangle is up-pointing, if $\operatorname{ITT}(\mathrm{J})=2$, the first triangle is down-pointing. (The LOAD format is not used).

(2) The IT $(\mathrm{J})+2$ triangle vertex coordinates for the $\mathrm{J}^{\prime}$ th hand are 
entered with the LOAD format. The coordinates are ordered

from left to right. The coordinates falling on either

horizontal boundary of the band must form a monotonically

increasing sequence.

MM. Entering the $\mathrm{S}_{\mathrm{N}}$ Specification and Constants

No data is entered in this input section of the code when ISNOPT $=1$. (One set of $S_{N}$ constants for all groups, built-in $E_{N}$ set used.)

If the group-dependent $S_{N}$ option is used, two sets of integers are read into the code. One is to indicate the correspondence between $S_{N}$ sets and energy groups and one is to identify the $S_{N}$ sets that are to be used.

First the array IDSN is entered with the LOAD format. IGM integers are entered which must lie in the range $1 \leq \operatorname{IDSN}(I G) \leq N S N$ for $I G=1$ to $I G M$. These integers identify the $S_{N}$ sets (there are $S_{N}$ sets one through NSN) that go with each energy group.

The array IISN is then entered with the LOAD format. NSN integers are entered. If IISN(I) is positive, then the built-in constants are used for the I'th $S_{N}$ set, and the order is given by the value of IISN(I). If IISN(I) is positive, it must be even and lie in the range between 2 and 16. If IISN(I) is negative, then a user entered set of $\mathrm{S}_{\mathrm{N}}$ constants is used for the I'th $\mathrm{S}_{\mathrm{N}}$ set, and the total number of directions given by the absolute value of IISN(I). TRIDENT-CTR requires that there be at least one direction per $\mu-\eta$ quadrant, so the minimum number of directions that can be read is four.

The user supplied $S_{N}$ sets are entered in the same order as they are specified in the IISN array. The first set of $S_{N}$ constants entered corresponds to the first negative value of IISN, the second set of $S_{N}$ constants entered corresponds to the second negative value of IISN, and so on.

A user supplied quadrature set consists of three arrays of floating point numbers each entered with the LOAD format. There are MM numbers in each array, where MM = IABS (IISN(I)) . The arrays entered are:

(1) WGT, the $\mathrm{S}_{\mathrm{N}}$ quadrature weights,

(2) COSMU, the $S_{N} \mu$-cosine directions, and

(3) COSETA, the $S_{N} \eta$-cosine directions.

Considerable freedom is allowed in the input of quadrature sets, however, some restrictions are made. 
(1) The weights must be normalized such that

$$
\sum_{I=1}^{M M} \operatorname{WGT}(I)=1.0 \text {. }
$$

(2) There must be at least one direction or quadrature point in each $\mu$ - $\eta$ quadrant. (See Fig. 17.)

(3) When top or bottom reflecting boundary conditions are specified, the directions must be symmetrical about the $\mu$-axis.

(4) When left or right reflecting boundary conditions are specified, the directions must be symmetrical about the $\eta$-axis.

(5) When the special boundary condition is used, the directions must be symmetric about both the $\mu$ and $\eta$ axis.

(6) When $r-z$ geometry is specified, the method used to approximate the angular derivative requires the directions to be symmetric about the $\eta$-axis to maintain neutron conservation.

(7) When anisotropic scattering is used, the quadrature set represented by the directions and weights must correctly integrate the spherical harmonic polynomials through order ISCT.

\section{NN. Entering the Source Specification Arrays}

[These arrays are specified only if IEVT is zero.]

The first two arrays in this section serve to identify inhomogeneous source and boundary source groups. Each array, if entered, is an integer array of length IGM, and is entered with the LOAD format.

The inhomogeneous source identification array (IDQ) is entered only if NQG is greater than zero. The integers entered in the IDQ array must lie in the range from one to NQG.

The boundary source identification array (IDBS) is entered only if NBSG is greater than zero. The integers entered in the IDBS array must lie in the range from one to NBSG.

The IDBL array is an integer array containing triangle identification numbers for the left internal boundary source. There is a triangle ID for each band containing the left internal boundary source, and the source is treated as entering that triangle from the right side. Additional discussion of the IDBL array is in Sec. III.Q.

The IDBL array is entered only if IBLI is greater than zero. The array is 
entered with the LOAD format with JLT integers. JLT is defined by:

$$
\text { JLT }=\text { JLIT }-J L I B+1
$$

where JLIT and JLIB are entries on card three and are the band identification numbers of the top and bottom bands of the left internal boundary source.

The IDBR array is an integer array containing triangle identification numbers for the right internal boundary source. There is a triangle identification numbers for each band containing the right internal boundary source, and the source is treated as entering that triangle from the left side. Additional discussion of the IDBR array is in Sec. III.Q.

The IDBR array is entered only if IBRI is greater than zero. The array is entered with the LOAD format with JRT integers. JRT is defined by:

$$
\text { JRT }=\text { JRIT }- \text { JRIB + } 1
$$

where JRIT and JRIB are entries on card three and are the band identifcation numbers of the top and bottom bands of the right internal boundary source. 00. Entering the Cross Sections

There are four different options for reading cross sections into TRIDENT-CTR; standard LASL input, FIDO input, CCCC standard interface file ISOTXS, and special LASL interface file GPODXS. The FIDO folmat and GPODXS file may not be combined with the other options or with each other.

The total number of materials read (MIN) may be calculated as follows:

$$
\begin{aligned}
& \left.\begin{array}{l}
\text { MCR }>0 \\
\text { and } \\
\text { MTPS }>0
\end{array}\right\} \quad M I N=M C R+M T P S *(I S C T+1) \\
& \text { MCR }<0 \quad \text { MIN }=- \text { MCR } \\
& \text { MTPS }<0 \quad \text { MIN }=-\operatorname{MTPS} *(\text { ISCT }+1)
\end{aligned}
$$

In all cases the cross section blocks or tables have the structures describ ed in Sec. III.V.

For the LASL format, each anisotropic cross section block is considered a material. Each block is entered by first reading a title card on an $18 \mathrm{~A} 4$ format, then reading IHM*IGM floating point numbers on a $6 \mathrm{E} 12.5$ format. These numbers are not entered with the LOAD format. 
Cross section data can also be entered with the FIDO or ANISN cross section format. Details can be obtained from Ref. 13. Likewise the details of using the ISOTXS file and specification for the IDLIB array are covered in Ref. 12 . The IDLIB array is an integer array with MTPS position numbers read in with the LOAD format.

With the above three options, the cross section data is read into SCM in material blocks. All the material are read into $\mathrm{SCM}$, then reordered into an energy group ordering and written to LCM or disk. For problems with a large number of energy groups and materials, the entire MIN*IHM*IGM array may not fit into SCM. In these cases, the LASL code MIXIT may be used. MIXIT takes the same basic input as described above and on card 4, and produces a binary file, GPODXS that has the cross section data arranged in group order. This file is read by TRIDENT-CTR into SCM then rewritten into LCM one group at a time.

PP. Entering the Starting Fluxes

The starting flux guess can be read by TRIDENT-CTR either from a binary file, or set of binary files, or from card images in the basic input file.

The binary file input is part of the restart capability of TRIDENT-CTR, and files used are created by previous TRIDENT-CTR runs. TRIDENT-CTR will write or dump the currently calculated fluxes for late: restart under three circumstances: a user set time limit dump, a periodic dump, and a final dump. When making a flux dump, up to three binary flux files can be created, DUMP1, DUMP2, and DUMP3. The length of these files may be set by the user and should be set to allow storage of the flux dump on magnetic tape. The maximum size file that may be written to a magnetic tape is approximately one million words (a new clean uncut tape). Large Tokamak problems with anisotropic scattering can create flux files much larger than one million words. When making a flux dump, the code will sequentially create, and fill the dump files, truncating by group. Each file contains part of the spatial mesh specification for verification of the flux file in later use.

The user may save these files and later restart from them. To do so, the user must rename the files RTFLUX, ATFLUX, and RAFLUX. When restarting from one file, RTFLUX is used, when restarting from two files, RTFLUX and ATFLUX are used, and when starting from three files, RTFLUX, ATFLUX, and RAFLUX are used. (See Sec. III.G for ISTART options.)

In some cases, the user may have saved only the scalar flux on the final 
dump, for making edits at a later time. When restarting for edit purposes using only the isotropic flux, ISTART is set to a $-5,-6$, or -7 , and ISTOP is set to -1. The code will read in the isotropic flux, skip the computational section, and perform the edits in the output portion of the code.

Input of a starting flux guess may be made from card images within the basic input file. Four basic options are available for this input, and are selected by the ISTART parameter on card one. All entries are made with the LOAD format. When entering values for the spatial mesh, entries are made on a per band basis with the LOAD format. The band entry length depends upon the mesh generator option. When MSHOPT $=0$, (automatic mesh generator), the number of entries for a particular band (IT defined for use with the mesh generator, is a region of constant "card image" starting flux guess, constant distributed source, and constant material specification. For MSHOPT $=1$ (input all vertex coordinates), IT* is equal to three times the number of triangles on the band. A flux is entered for each vertex of each triangle.

For ISTART negative the card image input will be as discussed below, except only the scalar or isotropic component of the flux is entered.

For $\operatorname{ISTART}=0$, no $f l u x$ guess is entered, ali fluxes are set to zero, and the fission array values are set to 1.0 .

[Note: $\quad \mathrm{NM}=($ ISCT +1$) *($ ISCT +2$) / 2$ ]

For ISTART $=1$, a group spectrum is entered as NM sets of IGM floating point numbers. Each set of IGM Numbers is treated as a separate entry by the LOAD format (each set of IGM Numbers is terminated by a three). The appropriate flux moment at all points (vertices) in the spatial mesh in a particular energy group is set equal to that group's spectrum value.

For ISTART $=2$, the entire flux array is entered as IGM groups of JT bands of $\mathrm{NM}$ sets of IT*. Each set of IT* floating point numbers is treated as a separate entry by the LOAD format (each set of IT* numbers is terminated by a three). For each group, the flux is loaded on a per band basis, with all flux moments being loaded for a particular band before moving on to the next band.

For $\operatorname{ISTART}=3$, a group spectrum is loaded, then a base spatially distributed flux is loaded. The flux guess for the problem is then formed by the product of the group spectrum times the base spatial distribution. The flux for a particular group is formed by multiplying the base spatial distribution by that 
groups spectrum value. The group spectrum is entered as IGM floating point numbers with the LOAD format. The base spatial distribution is loaded as JT bands of NM sets of IT* floating point numbers. Each set of IT* floating point numbers is treated as a separate entry by the LOAD format (each set IT* numbers is terminated by a three).

QQ. Entering the Inhomogeneous Distributed Source

The distributed source is entered only if IEVT $=0$, NQG $>0$, and IQOPT $>0$.

The options for entering the distributed source are similar to those for entering a flux guess from card images. The option is set by IQOPT on card 1. All entries are made with the $\mathrm{L} O A D$ format. The band entry length depends upon the mesh generator option. When MSHOPT = 0 (automatic mesh generator), the number of entries for a particular band (IT*) is equal to the number of zones in that band. A zone, as defined for use with the automatic mesh generator, is a region of constant "card image" inputed starting flux guess, constant distributed source, and constant material specification. For MSHOPT $=1$ (input a1l vertex coordinates), IT* is equal to three times the number of triangles on that band. A source is entered for each vertex of every triangle.

[Note: NMQ $=($ IQAN+1)*(IQAN+2)/2]

For IQOPT $=1$, a group sp trum is entered as NMQ sets of NQG floating point numbers. Each set of NQG numbers is treated as a separate entry by the IOAD format (each set of NQG numbers is terminated with a three). The source moment at all points (vertices) in the spatial mesh, in a particular energy group is set equal to that group's spectrum value.

For IQOPT $=2$, the entire distributed source is entered as NQG groups of JT bands of NMQ sets of IT*. Each set of IT $*$ floating point numbers is treated as a separate entry by the LOAD format (each set of IT* numbers is terminated by a three). For each group the source is loaded on a per band basis, with all source moments being loaded for a particular band before moving on to the next band.

For $\underline{I Q O P T}=3$, a group spectrum is loaded, then a base spatially distributed source is loaded. The distributed source for the problem is then formed by the product of the group spectrum times the base spatial distribution. The source for a particular group is formed by multiplying the base spatial distribution by that groups spectrum value. The group spectrum is entered as NQG floating point numbers with the LOAD format. The base spatial distribution is loaded as JT bands of. NMQ sets of IT* floating point numbers. Each set of IT* floating point 
numbers is treated as a separate entry by the LOAD format (each set of IT* numbers is terminated by a three).

RR. Entering the Boundary Sources

Boundary sources are entered only if IEVT $=0$ and NBSG $>0$.

The boundary sources are loaded as incoming angular fluxes rather than spherical harmonics moments. For side boundaries (left, left internal, right, and right internal) angular sources are loaded for every quadrature direction and the code determines which directions are incoming and uses only the angular sources for those directions. For the horizontal boundaries (top, top internal, bottom, and bottom internal) it is known a priori which directions are incoming. For example, for the top boundary only these quadrature directions in the second and third quadrants of the $\mu-\eta$ plane, $(\mu,-\eta)$ and $(-\mu,-\eta)$, are incoming. Hence for the horizontal boundaries either all the upward directed angular sources or all the downward directed angular sources are loaded.

The boundary sources are loaded on an energy group basis, until all NBSG groups have been loaded. The boundary source arrays within a given group are loaded in the following order:

(1) left boundary - BL array

(2) left internal boundary - BLI array

(3) right boundary - BR array

(4) right internal boundary - BRI array

(5) top boundary - BT array

(6) top internal boundary - BTI array

(7) bottom boundary - BB array, and

(8) bottom internal boundary - BBI array.

Before discussing the loading of data for specific boundary source arrays, some comments will be made on quadrature set structure and parameters. Since the boundary source is input as incoming angular fluxes, then the boundary source entries must correspond to the quadrature set used. Since the quadrature set may be group dependent, then the boundary source input may also vary with the energy group.

For a given quadrature set, the NPQ array gives the number of points per $\mu$ - $\eta$ quadrant, as shown in Fig. 18. The source arrays are loaded by quadrant, and MNPQ values must be entered for each quadrant. For quadrants where NPQ is less than MNPQ, the code only uses the first NPQ values entered for that quadrant. MNPQ and the NPQ array can be group dependent. 


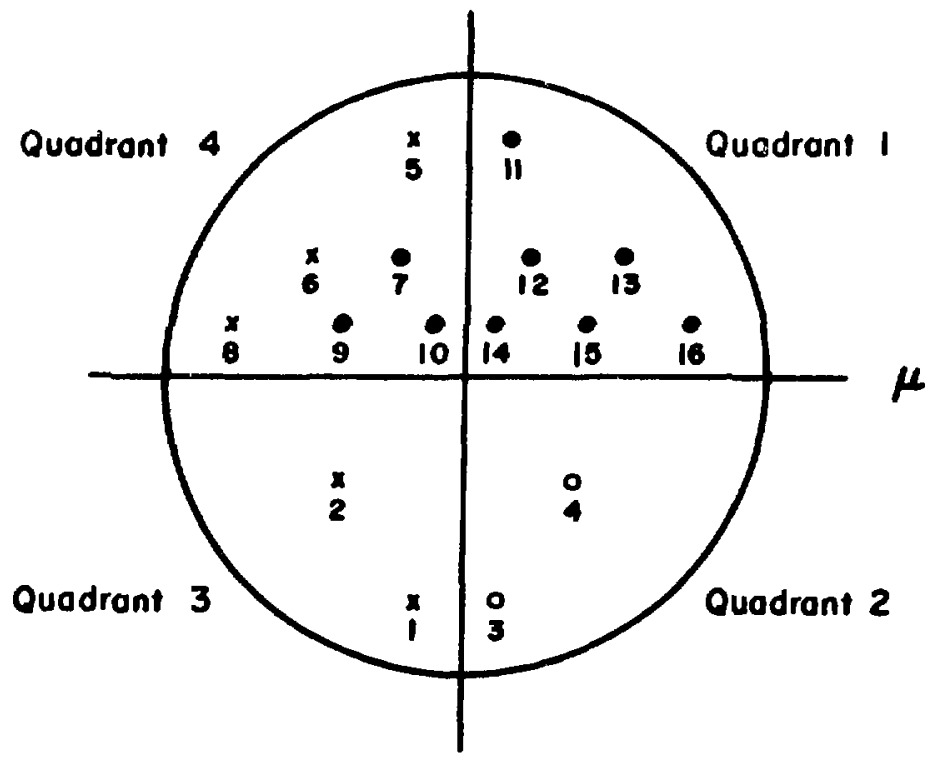

Fig. 17. Sample input quadrature set.

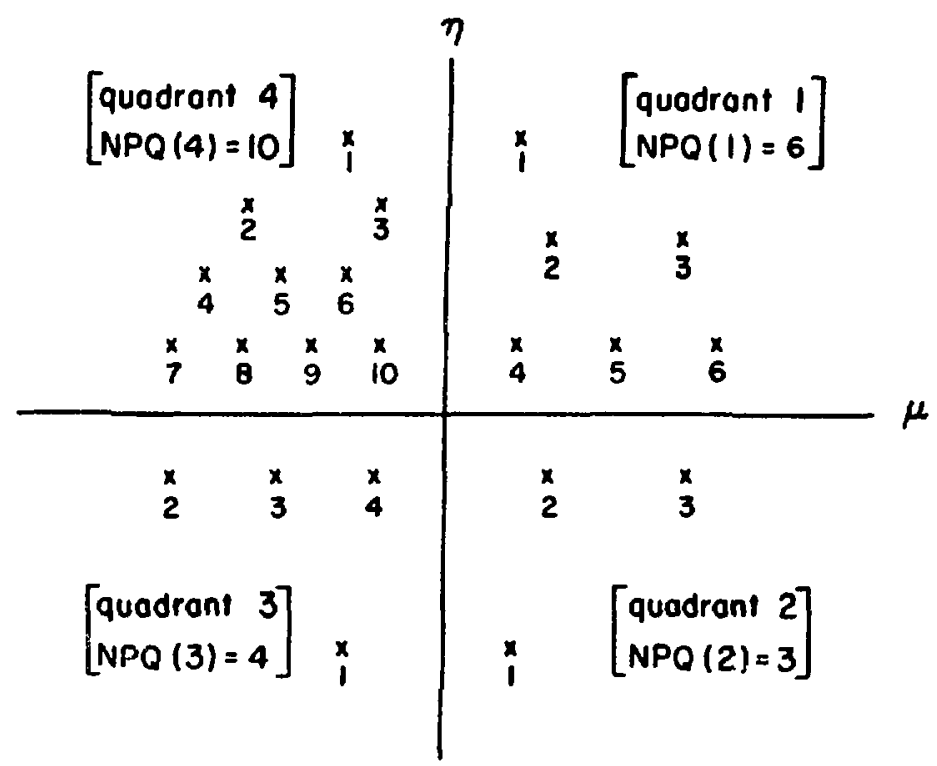

Fig. 18. Illustrative quadrature set. 
The ordering of the quadrature points within a $\mu$ - $\eta$ quadrant is indicated in Fig. 18. The points are ordered first on $\eta$-levels, with decreasing absolute values of $n$. The points are then ordered within the $\eta$-levels by increasing $\mu$ values.

The left boundary source is entered only if the value entered on card two for IBL is \pm 3 . The left houndary source, for a group, is loaded as a single entry with the LOAD format as an array of length $4 * M N P Q * J T L M B L$. LMBL $=1$ for IBL $=-3$ and LMBL $=2$ for IBL $=3$. The ordering of the array is indicated by the following implied DO loop:

$$
\mathrm{BL}_{k, j, m} ; k=1, \mathrm{LMBL} ; \mathrm{j}=1, \mathrm{JT} ; \mathrm{m}=1,4 \mathrm{MNPQ} .
$$

where the indices are cycled from inside out ( $k$ first, $j$ second, and $m$ last). The index $m$ is cycled through the quadrants in ascending order, and through the directions in each quadrant as indicated in Fig. 18.

The left internal boundary source is entered only if the value entered on card two for IBLI is gerater than zero. The left internal boundary source, for a group, is loaded as a single entry with the LOAD format as an array of length 4*MNPQ*JLT IBLI. JLT is the number of bands containing the left interior boundary source and is computed by:

$$
\mathrm{JLT}=\mathrm{JLIT}-\mathrm{JLIB}+1
$$

where JLIT and JLIB are the band identification numbers for the top and bottom bands of the left internal boundary source. JLIT and JLIB are entries on card 3 (see Sec. III.Q). The ordering of the array is indicated by the following implied Do loop:

$$
\mathrm{BLI}_{k, j, m} ; \mathrm{k}=1, \mathrm{IBLI} ; \mathrm{j}=1, \mathrm{JLT} ; \mathrm{m}=1,4 * \mathrm{MNPQ}
$$

where the indices are cycled from inside out ( $k$ first, $j$ second, and $m$ last). The index $\mathrm{m}$ is cycled through the quadrants in ascending order, and through the directions in each quadrant as indicated in Fig. 18.

The right boundary source and the right internal boundary are read in precisely the same manner as the left and left internal boundary sources. In the above discussion substitute IBR for IBL, LMBR for LMBL, IBRI for IBLI, JRT for JLT, JRIT for JLIT, and JRIB for JLIB. 
The top boundary source is entered only if $I B R= \pm 3$. The top boundary source, for a group, is loaded as two entries with the LOAD format. Only angular source values corresponding to downward directed quadrature points in quadrants 2 and 3 of the $\mu-\eta$ plane are entered. First the angular source for the inward or $-\mu$ directions in quadrant 3 are entered with the LOAD format as an array of length MNPQ:LMBT*NDNT. LMBT is one for IBT $=-3$ and LMBT is two for IBT $=3$. NDNT is the number of down-pointing triangles on the top band. The ordering of the array is as indicated by the following implied bo loop:

$$
\mathrm{BT}_{\text {(in) }, \mathrm{k}, \mathrm{i}} ; \mathrm{m}=1, \mathrm{MNPQ} ; \mathrm{k}=1, \mathrm{LMBT} ; \mathrm{i}=1 \text {, NDNT }
$$

where the indices are cycled from inside out ( $m$ first, $k$ second, and $i$ last). $m$ is cycled through the third quadrant as indicated in Fig. 18. The outward or $+\mu$ directions are then entered with the LOAD format in precisely the same fashion.

The top internal boundary source is entered only if IBTI is greater than zero. It is entered as a single entry with the LOAD format as an array of length $2 * M N P * 1 B T I * N B T I . ~ N B T I$ is the number of up-pointing triangles containing the top internal boundary source (see Sec. III.Q). The ordering of the array is an indicated by the following implied Do loop:

$$
\mathrm{BTI}_{m, k, i} ; m=1,2 \div \mathrm{MNPQ} ; \mathrm{k}=1, \mathrm{IBTI} ; \mathrm{i}=1, \mathrm{NBTI}
$$

where the indices are cycled from inside out (m first, $k$ second, and $i$ last). The index $m$ is cycled through the directions in the fourth quadrant first, then the first quadrant. Within the quadrants, $m$ follows the ordering shown in Fig. 18 .

The bottom boundary source is entered precisely as the top boundary source, except the directions in quadrant 4 are considered the inward directions and the directions in quadrant 1 are the outward directions. Substitute IBB for IBT, LMBB for LMBT, and NUPB for NDNT. NUPB is the number of up-pointing triangles on the bottom band.

The bottom internal boundary source is entered precisely as the top internal boundary source except quadrant 3 and 2 are used to order the input array BBI (first the sources corresponding to quadrant 3, then those corresponding to quadrant 2 are read in). Substitute IBBI for IBTI and NBBI for NBTI. 
SS. Entering the Material Identification Array (IDCS)

The material identification array (IDCS array) associates each triangle or mesh generator zone with a cross section block. The array is entered on a band by band basis with the LOAD format. Each band is treated as a separate entry by the LOAD format and the data entered for each band must be terminated with a 3 . The number of integer naterial identification numbers entered for each band is dependent upon the mesh generator options. When MSHOPT $=0$ (automatic mesh generator), the number of entries for a particular band, (IT\%), is equal to the number of zones in that band. A zone, as defined for use with the automatic mesh generator, is a region of constant "card image" input starting flux guess, constant distributed source, and constant material specification. For MSHOPT $=1$ (input all vertex coordinates), IT is equal to the number of triangles in that band.

If material ID's are negative, an anisotropic scattering source is calculated in that zone or triangle. The numbers need not be negative when ISCT $>0$.

It is assumed in TRIDENT-CTR that blocks of anisotropic cross sections which are used in the calculation have block numbers in ascending order starting with the isotropic cross section block. The entry in the IDCS array must be the block number for the isotropic cross section block. The isotropic cross section block ID's will be spaced every ISCT +1 integers apart starting with \pm 1 . For ISCT $=3$, for example, the allowable entries in the IDCS array will be $\pm 1, \pm 5, \pm 9, \pm 13, \ldots$. TT. Entering Miscel laneous Group Dependent Arrays

(1) Fission Fraction Array ( $\mathrm{CHI}$ ). The CHI array contains IGM numbers. Each floating point number gives the fraction of all particles per fission emitted in its corresponding energy group. The array is entered with the LOAD format. Although the fission fractions may depend upon the fissioning species and spatial position, TRIDENT-CTR does not allow these possibilities.

(2) Group Speed Array (VEL). The elements of the group speed array contain the neutron speeds associated with each group. The VEL array is always entered, but is only used in time-absorption calculations (IEVT $=2$ ). If IEVT $=2$, the value EV/VEL $(G)$ is added to the absorption and total cross sections in each group. The array of IGM floating point numbers is loaded with the LOAD format.

(3) Fictitious-Source Group Constant Array (FSG). The FSG array is loaded only if IFS $=1$. IFS is an entry on card one and is 
discussed in detail in Sec. III.E. The elements of the FSG array are actually the $\mathrm{Bg}^{\prime} \mathrm{s}$ of Sec. III.E. IGM floating-point numbers on the range zero to one are entered with the LOAD format.

UU. Entering the Cross Section Mixing Arrays (MIXNUM, MIXCOM, and MIXDEN)

These arrays are entered only if MS (entry four on card four) is greater than zero. The purpose and use of each array is discussed in Sec. III.U. Each array is entered separately with the LOAD format. MIXNUM and MIXCOM are integer arrays and MIXDEN is a floating point array.

VV. Entering the Buckling Height Array (BHT)

As discussed in Sec. III.FF, the buckling height array is used to simulate a third dimension with 'IRIDENT-CTR. The number of entries made is determined by the ISDB (entry nine on card six) and MSHOPT (entry eleven on card two) parameters. If $I S D B=0$ (whole system buckling height), one floating point number is entered with the LOAD format. If zero is entered, no buckling height correction is made. If ISDB $=1$ (space dependent buckling height), then JT bands of IT* floating point numbers are entered. The value of IT* for a certain band is determined by the mesh generator option. For MSHOPT $=0$ (automatic mesh generator), IT $*$ is equal to the number of mesh generator zones on the band. For MSHOPT = 1 (input all vertex coordinates), IT\% is equal to the number of triangles on the band. Each band is entered separately with the LOAD format.

WW. Entering the Edit Input

In addition to a standard output package, TRIDENT-CTR allows calculation and printed output of a variety of solution dependent data. A description of the information available from the edit portion of TRIDENT-CTR is contained in Sec. III. CC. The edit input is entered only if IEDOPT (entry two on card six) is equal to one. The first information entered for the edit input are the control integers NZEDS and NPEDS. These are respectively the number of zone edits to be made and the number of point edits to be made. NZEDS and NPEDS are read from a card image on a 216 format (not the LOAD format).

Next, NZEDS blocks of data are read, one block for each zone edit required. At the front of each zone edit data block, four control integers are read from a card image on a 416 format (not the LOAD format). The zone edit control integers are, in order, NZ, NCA, NMA, and NORMZ. NZ is the number of zones into which the spatial domain will be separated. Balance table quantities and desired constitu- 
ents and microscopic activity information will be printed for each zoute. NCA and NMA are the number of constituent and microscopic activities to be calculated and printed for each zone. The definition of constituent and microscopic activities is contained in Sec. III.CC.

Following the zone edit control integer card in each zone edit data block, entries are made with the LOAD format. An entry of NCA cross section ID's for the constituent activities is made if NCA is greater than zero. Next, NMA cross section ID's are entered if NMA is greater than zero. These cross section identification numbers are positive integers and must correspond to the isotropic cross section blocks. The zone identification numbers are entered as JT blocks of IT*. For MSHOPT $=0$ (automatic mesh generator), IT* for a particular band is equal to the number of mesh generator zones on that band. For MSHOPT $=1$ (input all vertex coordinates), IT $*$ for a particular band is equal to the number of triangles on that band. Each block of IT* is entered as a separate entry with the LOAD format. The zone identification numbers must be integers greater than zero and less than $\mathrm{NZ}$.

Once the zone edit data blocks have been entered, NPEDS blocks of data are entered, one block for each point edit desired. At the rront of each point edit, tinree control integers are read from a card image on a 316 format (not the LOAD format). These control integers are, in order, NTPE, NJPE, and NPMA. If NTPE is zero, all triangles are included in the point edit and further entry of mesh information is not required. If NTPE is greater than zero, then not all triangles are included in the point edit and identification of triangles to be included will be made on a per band basis. If NTPE is greater than zero, then NJPE is the number of bands that contain triangles included in the point edit. NPMA is the number of microscopic activities to be calculated in the point edit. Definition of lise microscopic point edit activity is provided in Sec. III.CC.

Following the point edit control integer card, data is entered with the LOAD format. If NTPE and NPMA are both zero, no additional data will be entered. If NMPA is greater than zero, then NMPA cross section ID's will be entered with the LOAD format. These cross section identification numbers are positive integers and must correspond to isotropic cross section blocks. If NTPE is zero, no further entries are made in this particular point edit data block. IF NTPE is greater than zero, NJPE band identification numbers are loaded with the LOAD format. These integers must be greater than zero and less than JT. Next NJPE 
blocks of IT* integers are entered with the LOAD format. For MSHOPT $=0$ (automatic mesh generator), IT* for a particular band is equal to the number of mesh generator zones on that band. For MSHOPT $=1$ (input all vertex coordinates), IT* for a particular band is equal to the number of triangles for that band. The integers must be equal to zero or one. If an integer is equal to zero, then the corresponding mesh generator zone or triangle will not be included in the point edit. For a value of one, it will be included.

XX. Entering the Plot Edit Input

An option of TRIDENT-CTR allows the creation of up to three binary files that may be post processed to produce three-dimensional graphical output. The option may be employed by setting IEDPLT (card five, entry seven) to one and entering the appropriate data in this section of the input. As indicated in Sec. III.AA, plots of the material composition, the fluxes, and various response functions may be made vs spatial position.

In all three types of plots, the variations may be shown for the entire spatial mesh or for a specified rectangular subregion. Entries in the BOUND array control the region displayed graphically. When entered, four floating point numbers are read into the BOUND array, XMIN, XMAX, YMIN, and MMAX. These numbers correspond to the minimum and maximum coordinates on the $x$ (or $r$ ) and $y$ (or z) axes to be plotted. The values read in are used to define the plotting domain, except in two cases. If both the minimum and maximum coordinates for an axis are equal or define a negative plotting area, the minimum and maximum values defined by the problem are used. If a coordinate extreme on a particular axis lies outside the problem domain, the corresponding extreme defined by the problem is used.

Data should be entered in this section only if IEDPLT $=1$.

The first information entered for the plot edit is a set of five plot edit control integers on a 516 format (not the LOAD format). The first three integers are, in order, the number of material plot edits (NMTPLT;, the number of flux plot edits (NFXPLT), and the number of response function plot edits (NRFPiT). The last two entries are NX and NY, the number of evenly spaced plotting divisions along the $x(o r r)$ and $y(o r z)$ axes. The 3-D plotting routine used in the current post processor, plots on an evenly spaced rectangular grid. If the values entered for $N X$ and $N Y$ are less than 1 , the code defaults them to one hundred. 
Next, NMTPLT blocks of data are entered, one block for each material plot edit. At the front of each material plot edit block, is a material plot edit integer control card with a single entry, NMT, read off a 1 I6 format. NMT is the number of materials to be included in the plot edit. Following this card, NMT cross section material identification numbers (IDMA array) are read in with the LOAD format. These integers must be positive and correspond to isotropic cross section blocks. The four element BOUND array, containing XMIN, XMAX, YMIN, and YMAX, is then entered with the LOAD format.

After the material plot edits have been entered, NFXPLT blocks of data are entered, one block for each flux plot edit. Beginning each flux plot edit block, is a flux plot edit control card with a single entry, NFXG, read off a 116 format. NFXG is the number of flux energy groups to be plotted. A separate plot is produced for each group. If NFXG is equal to IGM, all groups are plotted. If NFXG is equal to IGM +1 , all groups and the group summed flux are plotted. If NFXG $\leq$ IGM the IGPLT array (flux group identification numbers) is then entered with the LOAD format. The entry should consist of NFXG positive integers. For NFXG $\geqq$ IGM no entries are required. The four element BOUND array, containing XMIN, XMAX, YMIN, and YMAX, is then entered with the LOAD format.

Finally, NRFPLT blocks of data are entered, one block for each response function plot edit. A set of three response function plot edit control integers is read off a 316 format at the beginning of each block. The entries are the number of flux groups to be used in the plot edit (NRFG), the number of materials to be used in the plot edit (NMT), and the cross section table position to be used in the edit (IPP).

At a particular grid point, the information plotted is the sum over the desired energy groups of the flux for that group and position times the specified cross section (the IPP table position in the stipulated cross section block), if it exists at the grid point.

NRFG group identification numbers (IGPLT array) are then entered with the LOAD format. No entry is made if NRFG = IGM. Next the IDMA array is entered with the LOAD format as NMT positive integers. These integers must correspond to isotropic cross section blocks. The four elements of the BOUND array, consisting of XMIN, XMAX, XMIN, and YMAX are then entered with the LOAD format.

\section{CODE STRUCTURE}

As discussed in Sec. I.C, TRIDENT-CTR is a three level overlayed code. In 
Fig. 19 the overall flow of TRIDENT-CTR is shown with the names of key subroutines A list of subroulines ordered by overlay is given in Table VII, and a brief description of each subroutines function is contained in Table VIII.

The three major arrays of TRIDENT-CTR are IA, RIA, and A. The A array contains the major variable arrays used in TRIDENT-CTR. The IA array contains the key integer constants, single interger variables, and pointers for the major variable arrays contained in A. The RIA array contains floating point constants and single floating point variables. The contents of the IA and RIA arrays are given in Table IX.

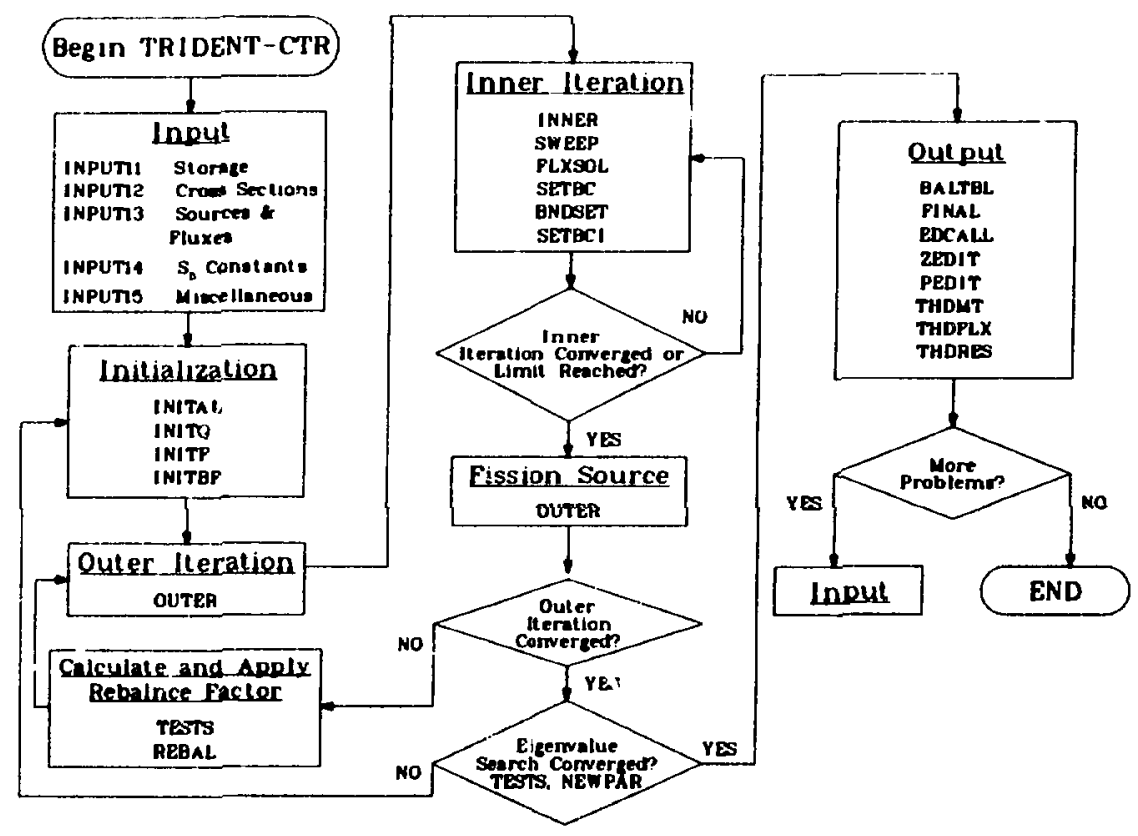

Eig. 19. TRIDENT-CTR program flow. 
TABLE VII

SUBROUTINES BY OVERLAY

\begin{tabular}{|c|c|c|c|}
\hline Overlay $(0,0)$ & Overlay $(1,0)$ & Overlay $(2,0)$ & Overlay $(3,0)$ \\
\hline TRDCTR & INPUT 10 & GRIND20 & OUTPT 30 \\
\hline 1. TRISSD & 1. IOAD & 1. REBAL & \\
\hline 2. OFFUGO & 2. FEBYTE & GRIND2 I & OUTPT31 \\
\hline 3. MONITR & INPUT II & 1. INITAL & 1. BALTBL \\
\hline 4. ERROR & 1. KSET & INITQ & FINAL \\
\hline 5. CLEAX & 2. POINT & 3. INIT & 3. FINALT \\
\hline 6. CLEAR & 3. SNLOAD & 4. INI'CBF & OUTPT32 \\
\hline 7. CLEAH & 4. POINTBF & 5. BFOJT & 1. EDCALL \\
\hline 8. MPLY & 5. XGEN & GRIND22 & ZEDIT \\
\hline 9. PRINTS & 6. VTXMV & 1. OUTER & PACT \\
\hline 10. DMPFLX & 7. XLOAD & INN:R & EDBF \\
\hline DREED & INPUT 12 & $A D S O R B$ & PEDIT \\
\hline LREED & 1. CSPREP & NEWAB & ARRMAP \\
\hline BFREED & 2. IFINXS & 5. SWEEP & 7. CONVRT \\
\hline CONVT & 3. RDGXS & 6. FLXSOL & OUTPT33 \\
\hline LSS & INPUT 13 & SETBC & $\begin{array}{ll}\text { 1. THDCNT } & \text { TH }\end{array}$ \\
\hline STNAA & 1. READQF & BNDCNST & 2. THDMT \\
\hline 17. SECONI & 2. READBS & 9. BNDSET & THDFLX \\
\hline 18. SREEI & 3. RDBND & 10. SETBCI & 4. THDRES \\
\hline 19. MCRED & 4. IFINQF & GRIND23 & LAGRN \\
\hline MORED & INPUT 14 & 1. TESTS & \\
\hline FILLU & 1. QUADCON & 2. NEWPAR & \\
\hline EILPU & 2. PRTSN & & \\
\hline 23. TIMER & 3. BCWHITE & & \\
\hline DOPCA & GENALP & & \\
\hline 25. DOPCTP & 5. SNCON & & \\
\hline BD1 & SORTQS & & \\
\hline SEEK & 7. IFINSN & & \\
\hline 28. REED & 8. PNGEN & & \\
\hline 29. SUBWD & 9. FSARY & & \\
\hline 30. SUBWR & 10. XKLGEN & & \\
\hline 31. LSRED & INPUT15 & & \\
\hline SHUTOF & 1. LOAD15 & & \\
\hline OPENRD & 2. LOADED & & \\
\hline 34. $\mathrm{BD} 2$ & 3. LDEDPT & & \\
\hline 35. DOPC & 4. GEOCON & & \\
\hline 36. CRED & 5. XPRINT & & \\
\hline 37. DRED & & & \\
\hline 38. DRIT & & & \\
\hline 39. LTSSHD & & & \\
\hline 40. REDUCE & & & \\
\hline 41. LTSAL & & & \\
\hline 42. LTSSCO & & & \\
\hline 43. LTSSCR & & & \\
\hline 44. LTSSDS & & & \\
\hline
\end{tabular}


TABLE VIII

FUNCTION OF TRIDENT-CTR SUBROUTINES

(Overlay) Subroutine

$(0,0) \quad \underline{\text { TRDCTR }}$

1. TRIDBD

2. OFFUGO

3. MONITR

4. ERROR

5. CLEAX

6. CLEAR

7. CLEAH

8. MPLY

9. PRINTS

10. DMPFLX

11. DREED

12. LREED

13. BFREED

14. CONVT

15. LSS

16. STNAA

17. SECONI

18. SREED

\section{Function}

Main driver of program. Initializes program; calls input, calculation, and output overlays; and attempts to execute sequential problems.

Initialize TRIDENT-CTR SEEK names and labels.

Finalizes files before exiting.

Prints resume of convergence parameters, monitor line headings, and outer iteration monitor data.

Prints messages, including error notices.

Sets a fixed-point Small-Core Memory (SCM) block to a constant.

Sets a floating-point SCM block to a constant.

Sets a Hollerith A6 block to a constant.

Multiplies an array by a constant.

Prints floating-point arrays.

Creates flux dump files and writes fluxes to disk for later restart.

Control routine for all random disk/LCM transfers.

Control routine for all banded LCM/SCM transfers.

Control routine for boundary flux LCM/SCM transfers.

Converts $M$-digit integer to character representation.

Linear-systems-of-equations solver.

Stores fixed-point value in floating-point array.

Initializes time function and obtains current time and data.

Control routine for binary operations other than random disk/LCM and LCM/SCM data transfers. 
TABLE VIII (cont)

(Overlay) Subroutine

19. MCRED

20. MDRED

21. FILLU

22. FILPU

23. TIMER

24. DOPCA

25. DOPCTP

26. BD 1

27. SEEK

28. REED

29. SUBRD

30. SUBWR

31. LSRED

32. SHUTOF

33. OPENRD

34. $\mathrm{BD} 2$

35. DOPC

36. CRED

37. DRED

38. DRIT

39. LTSSHD

40. REDUCE

\section{Function}

Control routine for all LCM/SCM transfers.

Control routine for random disk/LCM transfers.

Provides logical file information for DOPC.

Provides physical file information for DOPC.

Prepares system time and date.

Provides output and terminal units for $D O P C$ and SEEK series routines.

Prints random disk tabular information provided by DOPC.

Initializes disk parameters.

Assigns unit names and numbers for sequential binary files.

Performs all binary information transfers with the exception of random disk/LCM and LCM/SCM transfers.

Performs binary read of sequential files to SCM.

Performs binary write of seqeuential files from SCM.

Performs binary reads and writes between LCM and sequential disk.

Closes a binary device.

Renames a physical device.

Initializes disk parameters.

Assigns random disk space.

Performs LCM/SCM data transfers.

Performs random disk to LCM data transfers.

Performs $L C M$ to random disk data transfers.

Notifies when random disk transfer is complete.

Reduces physical file length. 


\section{TABLE VIII (cont)}

(Overlay) Subrout ine

41. LTSSAL

42. LTSSCO

43. LTSSCR

44. LTSSDS

$(1,0)$ INPUT 10

1. LOAD

2. FEBYTE

$(1,1)$ INPUT11

1. KSET

2. POINT

3. SNLOAD

4. POINTBF

5. XGEN

6. VTXMV

7. XLOAD

$(1,2)$ INPUT12

1. CSPREP

2. IFINXS

\section{Function}

Create a device with unit number and name with the file.

Open a device with unit number and name, when file is not present, create the file.

Create a disk file, destroy prior files with that name.

Destroy a file.

Calls five sections of this overlay.

Los Alamos data loader.

Converts 4-byte floating-point word to 8 -byte.

Reads basic parameter data, checks it, calculates storage locations in the container array and in bulk storage, prints basic parameter data.

Calculates integers NTC, NTX, NUPB, NDNT.

Calculates LCM pointers for use with LREED.

Loads group-dependent quadrature data.

Calculates LCM pointers for use with BFREED.

Load spatial mesh specifications and generates the spatial mesh when MSHOPT $=0$.

Relocates triangle vertices.

Loads spatial mesh by reading in all triangle vertex coordinates when MSHOPT $=1$.

Clears cross section, flux, and flow bulk storage. Calls CSPREP.

Reads cross sections in standard LASL format, FIDO format or from interface files by calling IFINXS or RDGXS. Prints cross sections, performs adjoint transpositions and reversals, checks cross sections, and calculates effective absorptions.

Interface input of cross sections from standard file ISOTXS. 
TABLE VIII (cont)

(Overlay) Subroutine

3. RDGXS

$(1,3)$ INPUT 13

1. READQF

2. READBS

3. RDBND

4. IFINQF

$(1,4)$ INPUT 14

1. QUADCON

2. PRTSN

3. BCWHITE

4. GENALP

5. SNCON

6. SORTQS

7. IFINSN

8. PNGEN

9. FSARY

10. YKLGEN

$(1,5)$ INPUT 15

1. LOAD15

2. LOADED

3. LDEDPT
Function

Interface input of cross sections from standard file GPODXS (group ordered).

Calls READQF to read source or flux guess.

Reads source or flux guess from cards or calls IFINQF to read source or flux guess from interface files. Prints flux if required.

Reads boundary source from cards. Calls RDBND.

Reads in top and bottom boundary sources.

Reads source or flux from standard-interface file. Calls QUADCON.

Controls calculation of $S_{N}$; and $S_{N}$ related constants.

Prints out $\mathrm{S}_{\mathrm{N}}$ constants.

Calculates constants for white boundary condition.

Generates angular derivative $(\alpha)$ coefficients.

Provides built-in library of $S_{N}$ constants, $S_{2}$ through $\mathrm{S}_{16}$.

Sorts input quadrature points into required sequence.

Interface input of $S_{N}$ constants from standard file SNCONS.

Generates spherical-harmonic ploynomials $R_{\ell}^{k}(\mu, \phi)$.

Generates coefficients needed for the ray-effectfix-up option.

Generates normalized spherical-harmonic polynomials $\mathrm{Y}_{\ell}^{\mathrm{k}}(\mu, \phi)$.

Calls LOAD 15 .

Controls loading of remaining data, including edit information. Generates spatial mesh plot file.

Loads edit data.

Loads plot edit data. 
TABLE VIII (cont)
(Overlay) Subroutine
Function
4. GEOCON
Calculates geometric constant arrays.
5. XPRINT
Prints out spatial mesh data.
$(2,0)$ GRIND20
1. REBAL
$(2,1)$ GRIND2 1
1. INITAL
Calls overlay $(2,1),(2,2)$, and $(2,3)$. Controls outer iteration process.
Calculates rebalance factor for both inner and outer iterations. Called by INNER and TESTS.
Calls INITAL, INITQ, and INITF.
Performs adjoint reversals of fission spectrum and group speeds, mixes cross sections, initializes fission spectrum, initializes source by call to INITQ, generates total cross section array, cal- culates fission source by call to INITF.
2. INITQ
Generates source rebalance information, normalizes sources and related quantities if necessary, prints sources if desired.
3. INITF Calculates and normalizes fission array.
4. INITBF Calculates total boundary source for a group.
5. BFOUT Prints out boundary snurces.
$(2,2)$ GRIND22 Calls OUTER
1. OUTER Calculates source to group and rebalance source, calls INNER, calculates new fission rate.
2. INNER Calculates total group source, performs sweep of spatial mesh by calls to SWEEP, checks for convergence of inner iterations, applies re- balance factors calculated by REBAL, accumu- lates outer-iteration-rebalance arrays.
3. ABSORB Computes total-absorption array for inner rebalance.
4. NEWAB Accumulates effective-absorption array for outer rebal ance.
5. SWEEP Performs sweep over the spatial mesh a band at a time, solves for angular flux on each triangle, stores outgoing flux for reflecting and special boundary conditions by call to STORBC, stores angular flux on last inner iteration if desired. 
TABLE VJII (cont)

(Overlay) Subrout ine

6. FIXXSOL

7. SETBC

8. BNDCNST

9. BNDSET

10. SETBCI

$(2,3)$ GRIND23

1. TESTS

2. NEWPAR

$(3,0)$ OUTPT30

$(3,1) \quad$ OUTPT31

1. BALTBL,

2. FINAL

3. FINALT

$(3,2) \quad$ OUTPT32

1. EDCALL

2. ZEDIT

3. PACT

4. EDBF
Funceion

Solves for the angular flux on the three triangle vertices given the source, cross section, and triangle dimensions.

Multiple entry-point routine to treat boundary conditions on the band sweeps.

Calculates the constants used for band interpolations.

Calculates the next band boundary flux.

Sets interior boundary source.

Calls TESTS.

Calculates rebalance factor for outer iteration acceleration, makes $k$ feductions, computes neutron balance, checks for outer convergence, applies rebalance factor and calls NEWPAR for eigenvalue searches.

Computes new parameters for implicit eigenvalue search.

Controls prints, interface-file output, and edits. Takes final dump.

Calls BALTBL FINAL, and FINALT.

Generates and prints neutron balance table at problem completion.

Prints scalar flux and moments and fission rate.

Prints scalar flux and moments at terminal if desired.

Calls EDCALL.

Performs zone edit by call to ZEDIT, performs point edit by call to PEDIT.

Calculates zone-balance tables, macroscopic activities, and power densities, calculates constituent and microscopic activities if desired.

Prints zone activities.

Computes the total boundary source for a group. 
TABLE VIII (cont)
(overlay) Subroutine
Function
5. PEDIT
Prints point scalar flux and moments computes microscopic activities if desired.
6. ARMAP
Draws map of point arrangement on a triangle.
7. CONVRT
Converts two-digit integer to character representa- tion. Used in ARRMAP.
$(3,3)$ oUTPT 33
Controls creation of binary files for plot edits.
1. THDCNT
Calculates constants used in 3-D plots and opens plot input file.
2. THDMT
Generates material plot binary file.
3. THDFLX
Generates flux plot binary file.
4. THDRES
Generates response function plot binary file.

TABLE IX

CONTENTS OF BLANK COMMON BLOCKS IA AND RIA

A. COMMON BLOCK IA

\begin{tabular}{|c|c|c|c|}
\hline Position & Name & Pointer for Array & Remarks \\
\hline 1 & ITH & & Theory. \\
\hline 2 & ISCT & & Scattering order. \\
\hline 3 & & & Not used. \\
\hline 4 & I GM & & Number of groups. \\
\hline 5 & & & Not used. \\
\hline 6 & JT & & Number of triangle bands. \\
\hline 7 & IBL & & Left-boundary specification. \\
\hline 8 & IBR & & Right-boundary specification. \\
\hline 9 & IBB & & Bottom-boundary specification. \\
\hline
\end{tabular}


TABI.F. IX (cont)

\begin{tabular}{|c|c|c|}
\hline Position & Pointer for Array & Remarks \\
\hline 10 & IBT & Top-boundary specification. \\
\hline 11 & IEVT & Eigenvalue-type specification. \\
\hline 12 & ISTART & Flux-input option. \\
\hline 13 & $\mathrm{MT}$ & Total number of materials. \\
\hline 14 & MIN & $\begin{array}{l}\text { Total number of input nuclides from both } \\
\text { library and cards. }\end{array}$ \\
\hline 15 & MS & Number of mixture instructions. \\
\hline 16 & IIT & Position in table of total cross sections. \\
\hline 17 & IHS & $\begin{array}{l}\text { Position in table of self-scatter cross } \\
\text { section. }\end{array}$ \\
\hline 18 & IHM & Cross section table length. \\
\hline 19 & IQOPT & Source-input options. \\
\hline 20 & IQAN & Distributed-source-anisotropy order. \\
\hline 21 & & Not used. \\
\hline 22 & & Not used. \\
\hline 23 & IPVT & $k_{e f f}$ or alpha-parametric-eigenvalue indicator. \\
\hline 24 & ISDB & System/fine-mesh buckling indicator. \\
\hline 25 & IGEOM & Geometry type. \\
\hline 26 & IITL & Maximum number of inner iterations. \\
\hline 27 & NSN & Number of sets of $\mathrm{S}_{\mathrm{N}}$ constants. \\
\hline 28 & MISN & Maximum $S_{N}$ order. \\
\hline 29 & MMNPQ & $\begin{array}{l}\text { Maximum number of } \mathrm{S}_{\mathrm{N}} \text { directions in a quadrant } \\
\text { in any } \mathrm{S}_{\mathrm{N}} \text { set. }\end{array}$ \\
\hline 31 & IEDOPT & Edit-option indicator. \\
\hline 32 & ISNOPT & $\mathrm{S}_{\mathrm{N}}$-input-option indicator. \\
\hline
\end{tabular}




\section{TABLE $1 X($ cont $)$}

\begin{tabular}{|c|c|c|}
\hline Posit rors & Pounter for Array & Remarks \\
\hline 33 & MSliop'T & Spatial-mesh-generator option indicator. \\
\hline 34 & $\operatorname{lns}$ & Ficlitious-source option indicator. \\
\hline 35 & NFSH & $\begin{array}{l}\text { Number of flux-moment derivatives in } \\
\text { fictitious source. }\end{array}$ \\
\hline 36 & MIM & Number of flux-moments in fictitious source. \\
\hline 37 & NǪG & Number of inhomogeneous source groups. \\
\hline 38 & NERROR & Input-error indicator. \\
\hline 39 & NEXTRR & Next-case indicator. \\
\hline 40 & I.ENUMP & Approxirite length of the dump. \\
\hline 41 & & Not used. \\
\hline 42 & & Not used. \\
\hline 43 & NBSG & Number of boundary source groups. \\
\hline 44 & IFXERIR & Integer print value for subroutine ERROR. \\
\hline 45 & JLDER & $\begin{array}{l}\text { Current subroutine LOAD field number or } \\
\text { count when exceeded. }\end{array}$ \\
\hline 46 & INLDER & Current subroutine LOAD card number. \\
\hline 47 & NCLDER & $\begin{array}{l}\text { Current subroutine LOAD number of required } \\
\text { entries in a block. }\end{array}$ \\
\hline 48 & & Not used. \\
\hline 49 & JBBI & $\begin{array}{l}\text { Band ID of the bottom internal boundary } \\
\text { source band. }\end{array}$ \\
\hline 50 & JBT I & $\begin{array}{l}\text { Band ID of the top internal boundary } \\
\text { source band. }\end{array}$ \\
\hline 51 & IUP & IHS-IHT-1 (upscatter indicator). \\
\hline 52 & IHF & IHT-1 (position of $v \sigma_{f}$ in table). \\
\hline 53 & IHA & IHT-2 (position of $\sigma_{a}$ in Table). \\
\hline 54 & IHTR & IHT-3 (position of $\sigma_{t r}$ in table if present). \\
\hline 55 & IHNN & IHT-4 (position of $\sigma_{n, 2 n}$ in table if p,resent). \\
\hline
\end{tabular}


TABLE IX (cont)

\begin{tabular}{|c|c|c|}
\hline Position & Pointer for Array & Remarks \\
\hline 56 & IMSHPT & Plot of spatial mesi option indicator. \\
\hline 57 & & Not used. \\
\hline 58 & NM & $\begin{array}{l}((\text { ISCT }+1) *(\text { ISCT }+2)) / 2 \text {, number of anisotropic } \\
\text { components of flux. }\end{array}$ \\
\hline 59 & NMQ & $\begin{array}{l}((I Q A N+1) *(I Q A N+2)) / 2 \text {, number of anisotropic } \\
\text { source moments. }\end{array}$ \\
\hline 60 & IEDPLT & Plot-edit-option indicator. \\
\hline 61 & ITZMAX & $\begin{array}{l}\text { Maximum number of mesh generator zones in } \\
\text { any one band. }\end{array}$ \\
\hline 62 & IGP & Sum IGM+1. \\
\hline 63 & NTC & Total number of triangles in system. \\
\hline 64 & ITMAX & Maximun number of triangles in any band. \\
\hline 65 & NTX & Total number of $x$-coordinates. \\
\hline 66 & NTF & Total number of space points. \\
\hline 67 & & Not used. \\
\hline 68 & NUPB & $\begin{array}{l}\text { Number of upward-pointing triangles on } \\
\text { bottom band. }\end{array}$ \\
\hline 69 & NDNT & $\begin{array}{l}\text { Number of downward-pointing triangles on } \\
\text { top band. }\end{array}$ \\
\hline 70 & & Not used. \\
\hline 71 & & Not used. \\
\hline 72 & IHMT & Product IHT*MT. \\
\hline 73 & & Not used. \\
\hline 74 & ISCP & Sum ISCT+1 \\
\hline 75 & I.NBT & Length of BT array. \\
\hline 76 & LNBB & Length of $\mathrm{BR}$ array. \\
\hline 77 & LNBL & Length of $\mathrm{BC}$ array. \\
\hline 78 & LNBR & Length of BR array. \\
\hline
\end{tabular}


TABLE IX (cont)

\begin{tabular}{|c|c|c|c|}
\hline Position & Name & Pointer for Array & Remarks \\
\hline 79 & ICLIM & & Length of the $C$ and AAJ blocks. \\
\hline 80 & LIT & $\mathrm{IT}(\mathrm{JT})$ & Number of triangles on each band. \\
\hline 81 & LITT & ITT (JT) & $\begin{array}{l}\text { Indicator for orientation of first tri- } \\
\text { angle on each band. }\end{array}$ \\
\hline 82 & LC & $\mathrm{C}(\mathrm{IHM}, \mathrm{MT})$ & Cross sections for a group. \\
\hline 83 & & & Not used. \\
\hline 84 & & & Not used. \\
\hline 85 & & & Not used. \\
\hline 86 & LITZ & $\operatorname{ITZ}(\mathrm{JT})$ & $\begin{array}{l}\text { Number of mesh generator zones on each } \\
\text { band. }\end{array}$ \\
\hline 87 & LQDAR & & $\begin{array}{l}\text { SCM pointer for beginning of quadrature } \\
\text { arrays. }\end{array}$ \\
\hline 88 & LQ & $Q(N M, 3$, I TMAX $)$ & Distributed source. \\
\hline 89 & $\mathrm{LX}$ & $X($ ITMAX +2$)$ & Radial coordinates of triangle vertices. \\
\hline 90 & LAE & $\mathrm{AE}(\operatorname{ITMAX}+1)$ & Area elements for a single band. \\
\hline 91 & LAS & AS (I'TMAX+2) & Sign of AE times 1.0 \\
\hline 92 & LAF & $\mathrm{AF}(3, \mathrm{ITMAX}+2)$ & Angular flux on a single band. \\
\hline 93 & ITBBI & & $\begin{array}{l}\text { Triangle ID of the first bottom internal } \\
\text { boundary source triangle. }\end{array}$ \\
\hline 94 & ITBTI & & $\begin{array}{l}\text { Triangle ID of the first top internal } \\
\text { boundary source triangle. }\end{array}$ \\
\hline 95 & LELEV & $\operatorname{ELEV}(\mathrm{JT}+1)$ & Elevation of horizontal band boundaries. \\
\hline 96 & & & Not used. \\
\hline 97 & LBLR & $\operatorname{BLR}(2, \mathrm{MNPQ})$ & $\begin{array}{l}\text { Left-reflecting-boundary-angular-flux } \\
\text { array. }\end{array}$ \\
\hline 98 & LFSG & FSG(IGM) & Fictitious source group constants $\left(\beta_{g}\right.$ 's) \\
\hline 99 & LFS & FS(NFSM, 4*MNPQ) & Fictitious source constants. \\
\hline 100 & LYI & YI (NYIM, 4*MNPQ) & $\begin{array}{l}\text { Constants to calculate fictitious source } \\
\text { angular moments. }\end{array}$ \\
\hline
\end{tabular}


TABLE IX (cont)

\begin{tabular}{|c|c|c|c|}
\hline Position & Name & Pointer for Array & Remarks \\
\hline 101 & LFSM & FSM(NFSM , 3, ITMAX) & $\begin{array}{l}\text { Current flux moment derivatives to calcu- } \\
\text { late ficitious source. }\end{array}$ \\
\hline 102 & LFSMA & FSMA (NFSM , 3, IMAX) & $\begin{array}{l}\text { Previous flux moment derivatives to calcu- } \\
\text { late fictitious source. }\end{array}$ \\
\hline 103 & LIP & $\operatorname{IP}(J T, 2,5+2 * \operatorname{IFS})$ & $\begin{array}{l}\text { LCM within-group pointer and length array for } \\
\text { transfer of banded arrays. }\end{array}$ \\
\hline 104 & LIPG & IPG $(5+$ IFS $)$ & Banded array group lengths. \\
\hline 105 & LFL & FLUX (NM , 3, ITMAX) & Angıllar moments of angular flux. \\
\hline 106 & NBBI & & $\begin{array}{l}\text { Number of source containing triangles in } \\
\text { bottom internal boundary source. }\end{array}$ \\
\hline 107 & NBTI & & $\begin{array}{l}\text { Number of source containing triangles in } \\
\text { top internal boundary source. }\end{array}$ \\
\hline 108 & IBC & & Boundary condition indicator. \\
\hline 109 & LIPBC & $\operatorname{IPBC}(2, \mathrm{IGM})$ & Boundary condition LCM pointer array. \\
\hline 110 & LLNBC & $\operatorname{LNBC}(2, \mathrm{IGM})$ & Boundary condition group length array. \\
\hline 111 & LDC & IDCS (ITMAX) & $\begin{array}{l}\text { Cross section identification number for } \\
\text { each triangle. }\end{array}$ \\
\hline 112 & IBS 1 & & Left and right boundary source indicator. \\
\hline 113 & IBS & & Boundary source indicator. \\
\hline 114 & LIDBS & IDBS ( IGM) & $\begin{array}{l}\text { Boundary source group identification } \\
\text { numbers. }\end{array}$ \\
\hline 115 & LIPBS & $\operatorname{IPBS}(5, \mathrm{NBSG})$ & Boundary source LCM pointer array. \\
\hline 116 & LLNBS & $\operatorname{LNBS}(5, \mathrm{NBSG})$ & Boundary source group length array. \\
\hline 117 & LPI & PI $(3, \operatorname{ITMAX})$ & $\begin{array}{l}\text { Geometric constants used for volume aver- } \\
\text { aging. }\end{array}$ \\
\hline 118 & LHY & HY (JT) & Band heights. \\
\hline 119 & & & Not used. \\
\hline 120 & LIDQ & $\operatorname{IDQ}(\mathrm{IGM})$ & $\begin{array}{l}\text { Distributed source group identification } \\
\text { numbers. }\end{array}$ \\
\hline 121 & & & Not used. \\
\hline
\end{tabular}




\section{TABLE IX (cont)}

\begin{tabular}{|c|c|c|c|}
\hline Position & Name & Pointer for Array & $\underline{\text { Remarks }}$ \\
\hline 122 & LHAF & $\operatorname{HAF}($ ISN $/ 2,3$, ITMAX) & $\begin{array}{l}\text { Half-angle angular flux for angular } \\
\text { derivatives. }\end{array}$ \\
\hline 123 & & & Not used. \\
\hline 124 & LP & $P(N M, 4 * M N P Q)$ & Spherical harmonic polynomials. \\
\hline 125 & & & Not used. \\
\hline 126 & & & Not used. \\
\hline 127 & & & Not used. \\
\hline 128 & LMN & $\operatorname{MN}(M S)$ & Input mixture numbers. \\
\hline 129 & LMC & MC(MS) & Input mixture instruction. \\
\hline 130 & LMD & $\mathrm{MD}(\mathrm{MS})$ & Input mixture densities. \\
\hline 131 & LF & $F(1)$ & Rebalance factor. \\
\hline 132 & LBM & $\operatorname{BM}(2,2,(\operatorname{ITMAX}+1) / 2)$ & $\begin{array}{l}\text { Interband interpolation constants for } \\
\text { angular flux. }\end{array}$ \\
\hline 133 & LDX & $\operatorname{IDX}((\operatorname{ITMAX}+1) / 2)$ & $\begin{array}{l}\text { Triangle identification numbers for inter- } \\
\text { band angular flux interpolation. }\end{array}$ \\
\hline 134 & & & Not used. \\
\hline 135 & LFLO & FLO $(3,1)$ & Whole system inward flows. \\
\hline 136 & LAB & $\mathrm{AB}(1)$ & Whole system absorption removal rate. \\
\hline 137 & LQQ & $Q Q(1)$ & Whole system source. \\
\hline 138 & & & Not used. \\
\hline 139 & & & Not used. \\
\hline 140 & & & Not used. \\
\hline 141 & & & Not used. \\
\hline 142 & IFP & & Flux-print option indicator. \\
\hline 143 & & & Not used. \\
\hline 144 & & & Not used. \\
\hline 145 & & & Not used. \\
\hline
\end{tabular}




\section{TABLE IX (cont)}

\begin{tabular}{|c|c|c|c|}
\hline Position & Name & Pointer for Array & Remarks \\
\hline 146 & LQG & QG(IGP) & Total integral of inhomogeneous source. \\
\hline 147 & LQGB & QGB (IGP) & Total integral of boundary source. \\
\hline 148 & IBT I & & Top internal boundary source indicator. \\
\hline 149 & IBBI & & Bottom internal boundary source indicator. \\
\hline 150 & IBLI & & Left internal boundary source indicator. \\
\hline 151 & IBRI & & Right internal boundary source indicator. \\
\hline 152 & LIDBL & IDBL (JLT) & $\begin{array}{l}\text { Triangle identification numbers for left } \\
\text { internal boundary source. }\end{array}$ \\
\hline 153 & LIDBR & IDBR(JRT) & $\begin{array}{l}\text { Triangle identification numbers for xight } \\
\text { internal boundary source. }\end{array}$ \\
\hline 154 & & & Not used. \\
\hline 155 & & & Not used. \\
\hline 156 & LBALTB & $\operatorname{BALTB}(9$, IGP $)$ & Balance table sums. \\
\hline 157 & LBAL & BAL(IGP) & Group reaction balance. \\
\hline 158 & LCHI & CHI (IGP) & Input fission spectrum. \\
\hline 159 & LCHIA & CHIA(IGP) & Fission spectrum used in the calculation. \\
\hline 160 & LVEL & $\operatorname{VEL}(\mathrm{IGP})$ & Group velocities. \\
\hline 161 & LIDSN & $\operatorname{IDSN}(\mathrm{IGM})$ & Group $\mathrm{S}_{\mathrm{N}}$ set identification numbers. \\
\hline 162 & LLNSN & LNSN (NSN) & Length of $S_{N}$ set block. \\
\hline 163 & LIPSN & $\operatorname{IPSN}(N S N)$ & LCM pointers for $S_{N}$ sets. \\
\hline 164 & & & Not used. \\
\hline 165 & & & ñot used. \\
\hline 166 & & & Not used. \\
\hline 167 & LAA & AAJ (MT) & Effective absorption cross section. \\
\hline 168 & LBHT & BHT (1) & Buckling height. \\
\hline
\end{tabular}




\section{TABLE IX (cont)}

\begin{tabular}{|c|c|c|c|}
\hline Position & Name & Pointer for Array & Remarks \\
\hline 170 & & & Not used. \\
\hline 171 & & & Not used. \\
\hline 172 & & & Not used. \\
\hline 173 & & & Not used. \\
\hline 174 & LCTOT & CTOT (ITMAX) & Effective total cross section. \\
\hline 175 & JCONV & & Final-convergence indicator. \\
\hline 176 & & & Not used. \\
\hline 177 & & & Not used. \\
\hline 178 & & & Not used. \\
\hline 179 & ICNT & & Iteration trigger used in NEWPAR. \\
\hline 180 & & & Not used. \\
\hline 181 & & & Not used. \\
\hline 182 & & & Not used. \\
\hline 183 & & & Not used. \\
\hline 184 & & & Not used. \\
\hline 185 & NGO & & Return indicator used in NEWPAR. \\
\hline 186 & & & Not used. \\
\hline 187 & & & Not used. \\
\hline 188 & IITNO & & Inner iteration limit. \\
\hline 189 & & & Not used. \\
\hline 190 & G & & Number of current group. \\
\hline 191 & ICONV & & Secondary-convergence indicator. \\
\hline 192 & NGOTO & & Return indicator set in TESTS. \\
\hline 193 & IAFW & & Angular-flux-file option indicator. \\
\hline 194 & & & Not used. \\
\hline
\end{tabular}


TABLE IX (cont)

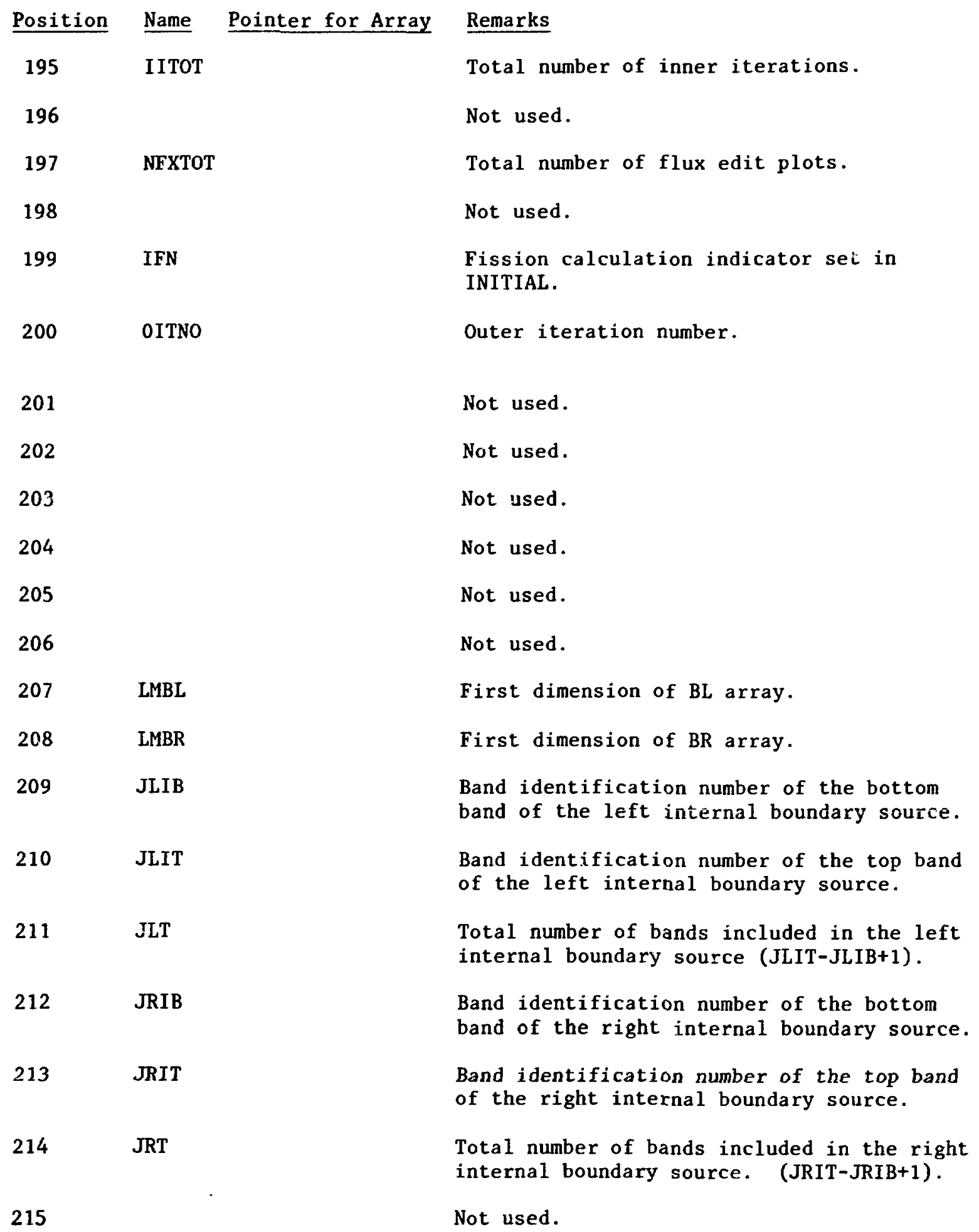




\section{TABLE IX (cont)}

\begin{tabular}{|c|c|c|c|}
\hline Position & Name & Pointer for Array & Remarks \\
\hline 216 & & & Not used. \\
\hline 217 & LYI 1 & YI 1 (NYIM, MNPQ) & First quadrant of YI constants. \\
\hline 218 & LYI2 & YI2(NYIM, MNPQ) & Second quadrant of YI contants. \\
\hline 219 & LYI3 & YI3(NYIM, MNPQ) & Third quadrant of YI constants. \\
\hline 220 & LYI4 & YI4 (NYIM, MNPQ) & Fourth quadrant of YI constants. \\
\hline 221 & LFS1 & FS1(NFSM, MNPQ) & First quadrant of FS constants. \\
\hline 222 & LFS2 & FS2 (NFSM, MNPQ) & Second quadrant of FS constants. \\
\hline 223 & LFS3 & FS3 (NFSM, MNPQ) & Third quadrant of FS constants. \\
\hline 224 & LFS4 & FS4 (NFSM, MNPQ) & Fourth quadrant of FS constants. \\
\hline 225 & IGGFX & & Within flux-block group index. \\
\hline 226 & IGGQ & & Within source block-group index. \\
\hline 227 & IGGXS & & Within cross section block group index. \\
\hline 228 & IGGFS & & Within fictitious source block group index. \\
\hline 229 & & & Not used. \\
\hline 230 & & & Not used. \\
\hline 231 & IGXSP & & Previous cross section group block. \\
\hline 232 & IGFXP & & Previous flux-group block. \\
\hline 233 & IGQP & & Previous source group block. \\
\hline 234 & IGFSP & & Previous fictitious-source group block. \\
\hline 235 & & & Not used. \\
\hline 236 & & & Not used. \\
\hline 237 & & & Not used. \\
\hline 238 & ISTOP & & Stop option indicator. \\
\hline 239 & IDUMP & & Flux dump option indicator. \\
\hline 240 & ITTY & & Terminal output option indicator. \\
\hline
\end{tabular}




\section{TABLE IX (cont)}

\begin{tabular}{|c|c|c|c|}
\hline Position & Name & Pointer for Array & Remarks \\
\hline 241 & IFILLI & & Flux dump file DUMP1 filled indicator. \\
\hline 242 & IFILL2 & & Flux dump file DUMP2 filled indcator. \\
\hline 243 & IFILL3 & & Flux dump file DUMP3 filled indicator. \\
\hline 244 & & & Not used. \\
\hline 245 & & & Not used. \\
\hline 246 & & & Not used. \\
\hline 247 & & & Not used. \\
\hline 248 & & & Not used. \\
\hline 249 & NOSGUP & & Sigma-up included in cross-section indicator. \\
\hline 250 & IOLYCS & & $\begin{array}{l}\text { Overlay indicator (first digit gives primary } \\
\text { overlay and second digit gives secondary } \\
\text { overlay when read in octal). }\end{array}$ \\
\hline 251 & ISN & & $\begin{array}{l}\text { Current group } S_{N} \text { order (when negative it } \\
\text { is the total number of directions in an } \\
\text { inputed } S_{N} \text { set). }\end{array}$ \\
\hline 252 & MNPQ & & $\begin{array}{l}\text { Current group maximum number of quadrature } \\
\text { points in any } \mu-\eta \text { quadrant. }\end{array}$ \\
\hline 253 & MNEL & & $\begin{array}{l}\text { Current group maximum number of eta levels } \\
\text { in any } \mu-\eta \text { quadrant. }\end{array}$ \\
\hline 254 & LCE & $\mathrm{CE}(\mathrm{MNPQ}, 4)$ & Axial direction cosines for current group. \\
\hline 255 & LCM & $\mathrm{CM}((\mathrm{MNPQ}, 4)$ & Radial direction cosines for current group. \\
\hline 256 & LW & W(MNPQ, 4) & Direction weights for current group. \\
\hline 257 & LP1 & $\mathrm{P} 1(\mathrm{NM}, \mathrm{MNPQ})$ & $\begin{array}{l}\text { First quadrant spherical harmonic poly- } \\
\text { nomials for current group. }\end{array}$ \\
\hline 258 & LP2 & P2(NM, MNPQ) & $\begin{array}{l}\text { Second quadrant spherical harmonic poly- } \\
\text { nomials for current group. }\end{array}$ \\
\hline 259 & LP3 & P3 (NM, MNPQ) & $\begin{array}{l}\text { Third quadrant spherical harmonic poly- } \\
\text { nomials for current group. }\end{array}$ \\
\hline 260 & IP4 & $\mathrm{P} 4(\mathrm{NM}, \mathrm{MNPQ})$ & $\begin{array}{l}\text { Fourth quadrant spherical harmonic poly- } \\
\text { nomials for current group. }\end{array}$ \\
\hline
\end{tabular}




\section{TABLE IX (cont)}

\begin{tabular}{|c|c|c|c|}
\hline Position & Name & Pointer for Array & Reinarks \\
\hline 261 & LAL1 & AL1 (MNPQ, 4) & $\begin{array}{l}\text { r-z geometry angular-derivative constants } \\
\text { for current group. }\end{array}$ \\
\hline 262 & LAL2 & AL2 (MNPQ,4) & $\begin{array}{l}r-z \text { geometry angular-derivative constants } \\
\text { for current group. }\end{array}$ \\
\hline 263 & LWE & WE $(M N P Q, 4)$ & $\begin{array}{l}\text { Quadrature weight times eta for current } \\
\text { group. }\end{array}$ \\
\hline 264 & LWM & WM(MNPQ, 4) & $\begin{array}{l}\text { Quadrature weight times mu for current } \\
\text { group. }\end{array}$ \\
\hline 265 & LWS & WS (3) & $\begin{array}{l}\text { White boundary condition weighted sums } \\
\text { for current group. }\end{array}$ \\
\hline 266 & LNUP & NUP (MNEL , 4) & $\begin{array}{l}\text { Number of directions on each eta level } \\
\text { for current group. }\end{array}$ \\
\hline 267 & LBR & $\mathrm{BR}(\mathrm{LMBR}, \mathrm{JT}, 4 \div \mathrm{MNPQ})$ & $\begin{array}{l}\text { Right-boundary angular flux array for } \\
\text { current group. }\end{array}$ \\
\hline 268 & LBNDI & $\begin{array}{l}\text { BNDI }(M N P Q, 2 * \\
((\operatorname{ITMAX}+1) / 2)\end{array}$ & $\begin{array}{l}\text { Incoming (right to left) angular flux } \\
\text { array on band boundary. }\end{array}$ \\
\hline 269 & LBNDS & $\begin{array}{l}\text { BNDO }(M N P Q, 2 * \\
((\operatorname{ITMAX}+1) / 2)\end{array}$ & $\begin{array}{l}\text { Outgoing (left to right) angular flux array } \\
\text { on band boundary. }\end{array}$ \\
\hline 270 & LBL & BL(LMBL , JT , 4MNPQ) & $\begin{array}{l}\text { Left boundary angular-flux array for } \\
\text { current group. }\end{array}$ \\
\hline 271 & LBLI & $\begin{array}{l}\text { BLI ( IBLI, JLT , } 4 * \\
\text { MNPQ) }\end{array}$ & $\begin{array}{l}\text { Left internal boundary source array for } \\
\text { current group. }\end{array}$ \\
\hline 272 & LBRI & $\begin{array}{l}\text { BRI ( IBRI , JRT , } 4 * \\
\text { MNPQ) }\end{array}$ & $\begin{array}{l}\text { Right internal boundary source array for } \\
\text { current group. }\end{array}$ \\
\hline 273 & LBHI & $\begin{array}{l}\text { BHI (MNPQ, } 2 * \text { NBTI }) \\
\text { or } \\
\text { BHI (MNPQ , } 2 * \text { NBBI })\end{array}$ & $\begin{array}{l}\text { Horizontal internal boundary source array } \\
\text { for current group. }\end{array}$ \\
\hline 274 & LNEL & $\operatorname{NEL}(4)$ & $\begin{array}{l}\text { Number of eta levels per } \mu-\eta \text { quadrant } \\
\text { for current group. }\end{array}$ \\
\hline 275 & LNPQ & NPQ (4) & $\begin{array}{l}\text { Number of directions per } \mu-\eta \text { quadrant } \\
\text { for current group. }\end{array}$ \\
\hline $\begin{array}{r}276 \\
\text { to }\end{array}$ & & & Not used \\
\hline
\end{tabular}


TABLE IX (cont)

B. COMMON BLOCK RIA

\section{Position Name}

$1 \quad$ EV

$2 \quad$ EVM

$3 \quad \mathrm{PV}$

4 XLAL

$5 \quad$ XLAH

6 XLAX

7 EPSO

8 EPSI

9 EPSR

10

11

12

13

14

15

16

17

18

19

20

21

\section{Remarks}

Eigenvalue guess.

Eigenvalue modifier.

Parametric value of $\mathrm{k}_{\mathrm{ef}}$ or alpha.

Search-lambda-lower limit .

Search-lambda-upper limit.

Fine-mesh-search precision.

Outer-convergence precision.

Inner-convergence precision $=$ EPSO .

Within-group-rebalance-convergence precision $=10^{4}$.

Whole-system-rebalance-convergence precision = EPSO.

Normalization amplitude.

New parameter modifier.

Weighted sum of mu over cutward $(\mu>0)$ directions.

Weighted sum of eta over upward $(\eta>\hat{u})$ directions.

Weighted sum of eta over downward $(\eta>0)$ directions.

Problem volume.

Not used.

Not used.

Not used.

$\mathrm{N}, 2 \mathrm{~N}$ reaction term used in balance equation.

Value of lambda from sequence of outer iterations previous to that of XLAP. 
TABLE IX (cont)

\begin{tabular}{|c|c|c|}
\hline Position & Jame & Renarks \\
\hline 22 & XLAP & $\begin{array}{l}\text { Value of lambda from previous sequence of } \\
\text { outer iterations. }\end{array}$ \\
\hline 23 & E2 & Temporary storage. \\
\hline 24 & E1 & Temporary storage. \\
\hline 25 & EVPP & $\begin{array}{l}\text { Eigenvalue from cycle of outer iterations } \\
\text { previous to that of EVP. }\end{array}$ \\
\hline 26 & EVP & $\begin{array}{l}\text { Eigenvalue from previous sequence of } \\
\text { outer iterations. }\end{array}$ \\
\hline 27 & $\mathrm{E} 4$ & Temporary storage. \\
\hline 28 & ALAR & $\begin{array}{l}\text { Value of lambda from previous outer itera- } \\
\text { tion. }\end{array}$ \\
\hline 29 & $\mathrm{TS}$ & Total integrated source to a group. \\
\hline 30 & 3 & Temporary storage. \\
\hline 31 & EVS & Slope used in eigenvalue search. \\
\hline 32 & ALA & Parameter lambda. \\
\hline 33 & TIN & $\begin{array}{l}\text { Time at the last dump (also used as tem- } \\
\text { porary storage. }\end{array}$ \\
\hline 34 & FT & $\begin{array}{l}\text { Total space-integrated fission source in a } \\
\text { group. }\end{array}$ \\
\hline 35 & FTP & Previous fission total. \\
\hline 36 & & Not used. \\
\hline 37 & & Not used. \\
\hline 38 & & Not used. \\
\hline 39 & & Not used. \\
\hline 40 & ERR & $\begin{array}{l}\text { Scalar-flux error for comparison with } \\
\text { previous flux. }\end{array}$ \\
\hline 41 & FLTERR & $\begin{array}{l}\text { Floating-point print value for subroutine } \\
\text { ERROR. }\end{array}$ \\
\hline 42 & TIMACC & Accumulated problem running time. \\
\hline
\end{tabular}




\section{TABLE IX (cont)}

\begin{tabular}{|c|c|c|}
\hline Position & Name & Remarks \\
\hline 43 & TIMOFF & $\begin{array}{l}\text { Floating-point form of the input fixed- } \\
\text { point parameter ITLIM. }\end{array}$ \\
\hline 44 & TIMSLD & Elapsed time since last dump. \\
\hline 45 & & Not used. \\
\hline 46 & & Not used. \\
\hline 47 & & Not used. \\
\hline 48 & & Not used. \\
\hline $4 y$ & & Not used. \\
\hline 50 & & Not used. \\
\hline 51 & & Not used. \\
\hline 52 & & Not used. \\
\hline 53 & & Not used. \\
\hline 54 & & Not used. \\
\hline 55 & & Not used. \\
\hline 56 & & Not used. \\
\hline 57 & & Not used. \\
\hline 58 & & Not used. \\
\hline 59 & & ivot used. \\
\hline 60 & CZERO & 0.0, IBM double precision. \\
\hline 61 & CONE & 1.0, IEM double precision. \\
\hline 62 & CTWO & 2.0, IBM double precision. \\
\hline 63 & CHALF & 0.5 , IBM double precision. \\
\hline 64 & CTOWPI & $2 * \pi$, IBM double precision. \\
\hline 65 & $\mathrm{CPI}$ & $\pi$, IBM double precision. \\
\hline 66 & RTOWPI & $1 /(2 * \pi)$, IBM double precision. \\
\hline 67 & & Not used. \\
\hline
\end{tabular}


TABLE IX (cont)

Position Name

68

69

70

$7 I$

72
Remarks

Not used.

Not used.

Not used.

Not used.

Not used. 


\section{REFERENCES}

1. T. J. Seed, W. F. Miller, w., and F. W. Brinkley, Jr., TRIDENT: A TwoDimensional, Multigrouf, Triangular Mesh Discrete Ordinates, Explicit Neutron Transport Code," Los Alamos Scientific Laboratory report LA-6735-M (March 1977).

2. K. D. Lathrop and F. W. Brinkley, "TWOTRAN-II: An Interfaced, Exportable Version of the TWOTRAN Code for Two-Dimensional Transport," Los Alamos Scientific Laboratory report LA-4848-MS (July 1973).

3. W. A. Rhoades and F. R. Mynatt, "DOT III Two-Dimensional Discrete-Ordinates Code," Oak Ridge National Laboratory report ORNL-TM-4280 (1973).

4. W. H. Reed, T. R. Hill, F. W. Brinkley, and K. D. Lathrop, "TRIPLET: A TwoDimensional Multigroup, Triangular Mesh, Planar Geometry, Explicit Transport Code," Los Alamos Scientific Laboratory report LA-5428-MS (1973).

5. K. D. Lathrop, "Discrete-Ordinates Methods for the Numerical Solution of the Transport Equation," Reactor Technology 15, 107 (1972).

6. W. F. Miller, Jr., and Wm. H. Reed, "Ray-Effect Mitigation Methods for TwoDimensional Neutron Transport Theory," Nucl. Sci. Eng. 62 (March 1977).

7. K. D. Lathrop, "Ray Effects in Discrete Ordinates Equations," Nucl. Sci. Eng. 32, 357 (1968).

8. W. F. Miller, Jr., and W. H. Reed, "Discrete Ordinates-to-Spherical Harmoric Conversions for Kay Effect Mitigation in $x-y$ Geometry," Proc. Conf. Computational Methods in Nuclear Engineering, Charleston, SC (April 15-17, 1975).

9. G. I. Bell, G. E. Hansen, and H. A. Sandmeier, "Multitable Treatments of Anisotropic Scattering in $\mathrm{S}_{\mathrm{N}}$ Multigroup Transport Calculations," Nucl. Sci. Eng. 28, 376 (1967).

10. K. D. Lathrop, "Remedies for Ray Effects," Nucl. Sci. Eng. 45, 255 (1971).

11. B. M. Carmichael, "Standard Interface Files and Procedures for Reactor Physics Codes, Version-III," Los Alamos Scientific Laboratory report LA-5486-MS (February 1974).

12. W. W. Engle, Jr., "A Users Manual for ANISN, A One-Dimensional Discrete Ordinates Transport Code with Anisotropic Scattering," Union Carbide Corporation report K-1693 (March 1967).

13. B. G. Carlson, "Tables of Symmetric Equal Weight Quadrature $\mathrm{EQ}_{\mathrm{N}}$ Over the Unit Sphere," Los Alamos Scientific Laboratory report LA-4734 ${ }^{\mathrm{N}}$ (1971). 